\title{
Skeletal muscle mechanics : a numerical and experimental approach to spatial phenomena
}

Citation for published version (APA):

van Donkelaar, C. C. (1999). Skeletal muscle mechanics : a numerical and experimental approach to spatial phenomena. [Doctoral Thesis, Maastricht University]. Universiteit Maastricht. https://doi.org/10.26481/dis.19990528cd

Document status and date:

Published: 01/01/1999

DOI:

$10.26481 /$ dis. $19990528 \mathrm{~cd}$

Document Version:

Publisher's PDF, also known as Version of record

\section{Please check the document version of this publication:}

- A submitted manuscript is the version of the article upon submission and before peer-review. There can be important differences between the submitted version and the official published version of record.

People interested in the research are advised to contact the author for the final version of the publication, or visit the DOI to the publisher's website.

- The final author version and the galley proof are versions of the publication after peer review.

- The final published version features the final layout of the paper including the volume, issue and page numbers.

Link to publication

\footnotetext{
General rights rights.

- You may freely distribute the URL identifying the publication in the public portal. please follow below link for the End User Agreement:

www.umlib.nl/taverne-license

Take down policy

If you believe that this document breaches copyright please contact us at:

repository@maastrichtuniversity.nl

providing details and we will investigate your claim.
}

Copyright and moral rights for the publications made accessible in the public portal are retained by the authors and/or other copyright owners and it is a condition of accessing publications that users recognise and abide by the legal requirements associated with these

- Users may download and print one copy of any publication from the public portal for the purpose of private study or research.

- You may not further distribute the material or use it for any profit-making activity or commercial gain

If the publication is distributed under the terms of Article $25 \mathrm{fa}$ of the Dutch Copyright Act, indicated by the "Taverne" license above, 


\title{
SKELETAL MUSCLE MECHANICS
}

\author{
A numerical and experimental approach \\ to spatial phenomena
}


Van Donkelaar, Corrinus Cornelis

Skeletal muscle mechanics,

a numerical and experimental approach to spatial phenomena

Proefschrift Universiteit Maastricht, 1999

ISBN 90-9012672-4

Druk: Print Partners Ipskamp 


\title{
A numerical and experimental approach to spatial phenomena
}

PROEFSCHRIFT

ter verkrijging van de graad van doctor aan de Universiteit Maastricht, op gezag van de Rector Magnificus, Prof. dr. A.C. Nieuwenhuijzen Kruseman, volgens het besluit van het College van Decanen,

in het openbaar te verdedigen op vrijdag 28 mei 1999 om 14.00 uur.

door

\section{CORRINUS CORNELIS VAN DONKELAAR}

\author{
geboren te Ermelo op 1 november 1970
}




\section{Promotores:}

Prof. dr. H. Kuipers

Prof. dr. ir. J.D. Janssen

Co-Promotor:

Dr. ir. M.R. Drost

Beoordelingscommissie:

Prof. dr. ir. M.G.J. Arts (voorzitter)

Dr. ir. J.M. Huyghe

Prof. dr. A. Huson

Dr. H.H.C.M. Savelberg

Prof. dr. D.W. Slaaf 
voor Nanda voor mijn ouders 



\section{TABLE OF CONTENTS}


1. INTRODUCTION 1

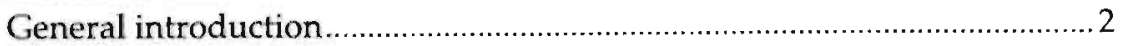

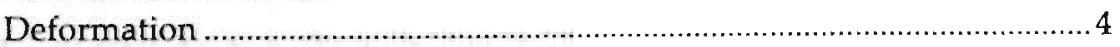

Muscle constitution and contraction .................................................... 4

Spatial interactions .

Perfusion

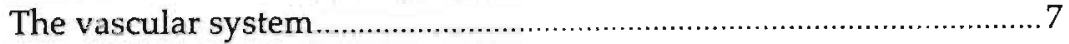

Perfusion in contracting striated muscle ............................................ 10

Modeling skeletal muscle perfusion......................................................... 11

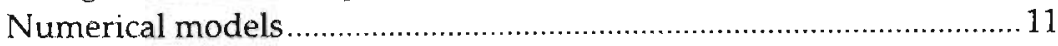

The finite element model of perfused, contracting skeletal muscle ....12

Model parameters vs. physiology ……................................................ 15

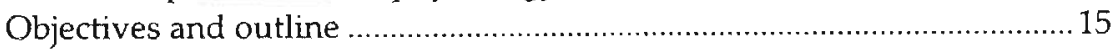

2. THE EFFECT OF CONTRACTION ON TOTAL MUSCLE PERFUSION 17

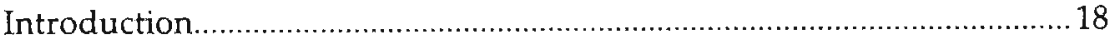

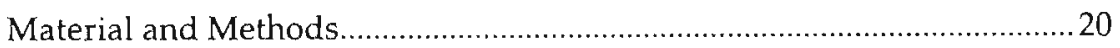

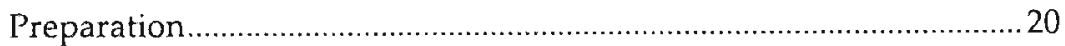

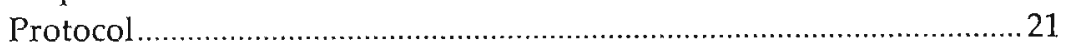

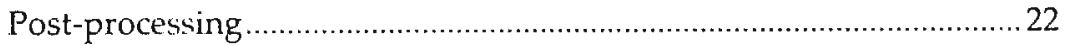

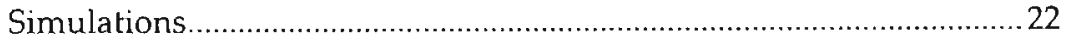

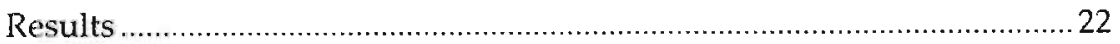

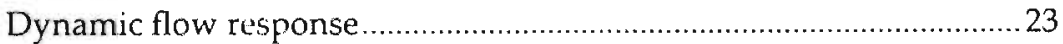

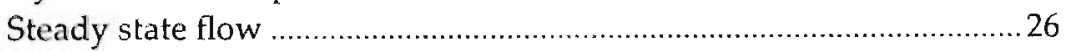

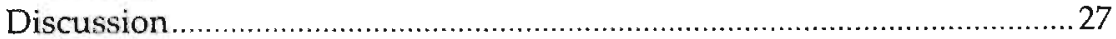

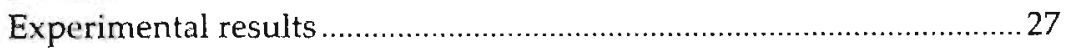

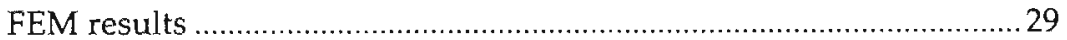

Experimental procedure

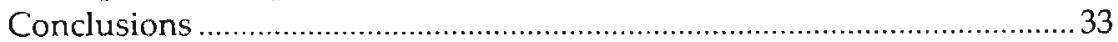

3. SPATIAL INTERACTION BETWEEN TISSUE PRESSURE AND MUSCLE PERFUSION DURING CONTRACTION 35

Introduction

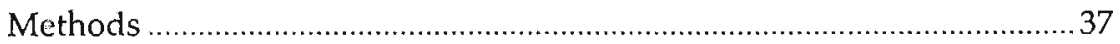

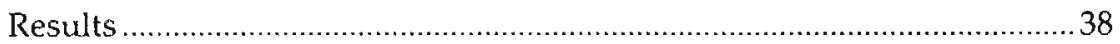

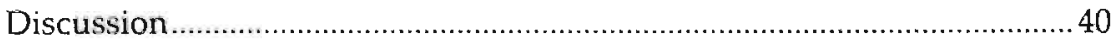

4. SKELETAL MUSCLE TRANSVERSE STRAIN DURING ISOMETRIC CONTRACTION AT DIFFERENT LENGTHS 43

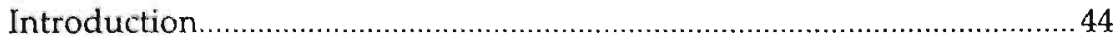

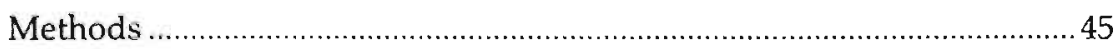

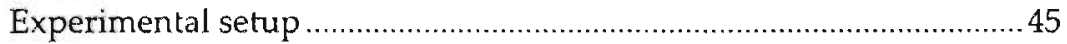

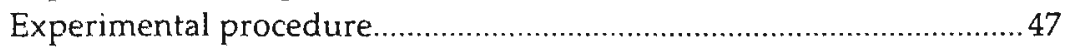

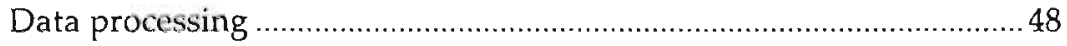

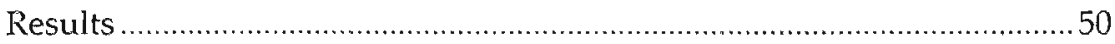


Discussion

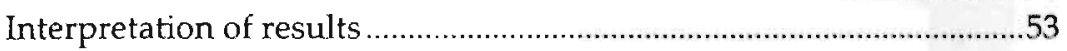

Experimental procedure ................................................................

5. THREE-DIMENSIONAL RECONSTRUCTION AND FINITE ELEMENT MESH GENERATION OF RAT CALF MUSCLE

Introduction

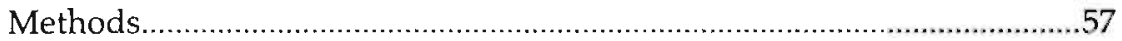

Triceps surae muscle reconstruction …….......................................57

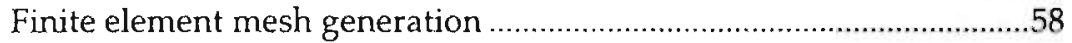

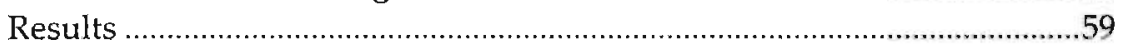

Triceps surae muscle reconstruction ...............................................59

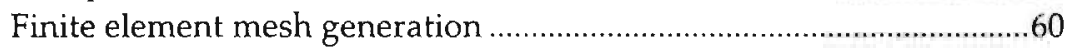

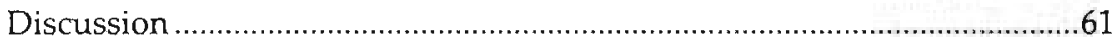

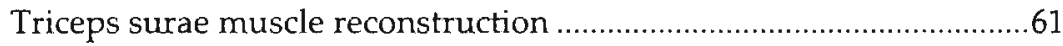

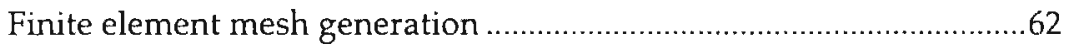

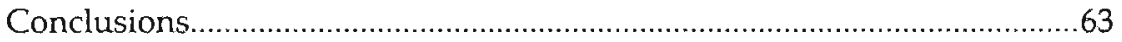

6. DIFFUSION TENSOR IMAGING IN BIOMECHANICAL STUDIES OF SKELETAL MUSCLE FUNCTION 65

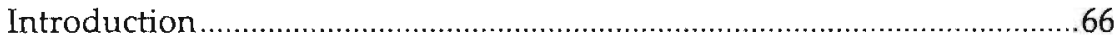

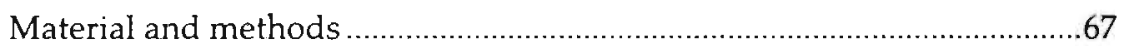

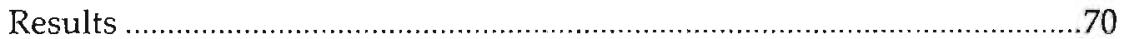

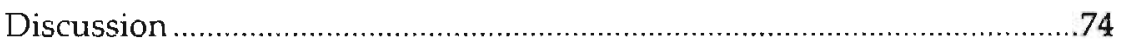

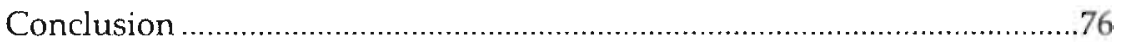

$\begin{array}{ll}\text { 7. DISCUSSION } & 79\end{array}$

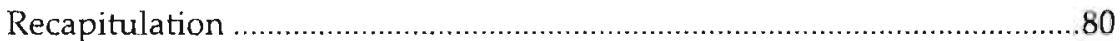

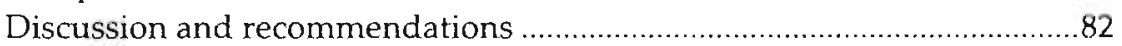

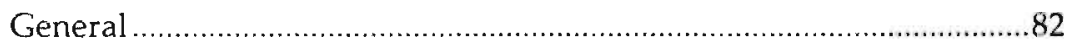

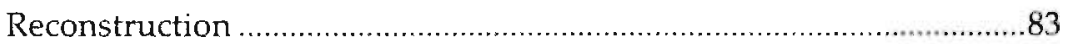

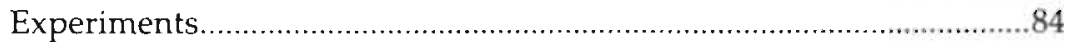

Model …………

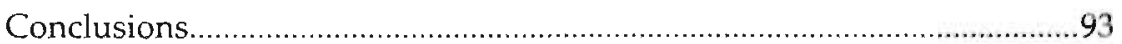

$\begin{array}{ll}\text { LITERATURE } & 94\end{array}$

$\begin{array}{ll}\text { SUMMARY } & 101\end{array}$

$\begin{array}{ll}\text { SAMENVATTING } & 104\end{array}$

$\begin{array}{ll}\text { PUBLICATIONS } & 107\end{array}$

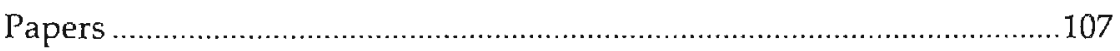

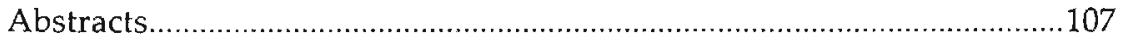

$\begin{array}{ll}\text { DANKWOORD } & 109\end{array}$ 



\section{INTRODUCTION}

\footnotetext{
A general introduction into skeletal muscle functioning, anatomy and perfusion is provided and research objectives are formulated. Muscle mechanics is studied with respect to deformation and perfusion during contraction, by a combination of experiments and a numerical model. An introduction to this model is captured as well.
} 


\section{GENERAL INTRODUCTION}

The ability to move is an important part of the quality of life. This becomes clear if one considers the impact of any form of (chronic) disease related to bones, articulations, skeletal muscles or the neuromuscular system on a men's life. To be able to understand the way movements are being performed, one must understand the functioning of each individual part of the locomotion apparatus. Skeletal muscles take a special place as they initialize all voluntary motions by their ability to change length and to generate well-defined forces at different lengths. To fulfill this task, they consist of a genius mechanism of billions of interacting filaments, supported by metabolic, neurological and biochemical processes that are still hardly understood. Many studies from different fields addressed skeletal muscle functioning in an effort to describe and understand parts of the complicated but intriguing muscle functioning:

As generating force at different lengths and velocities is a muscle's most obvious task, well-documented studies into mechanical muscle functioning date back to the $19^{\text {th }}$ century when Blix et al described a force-length relationship for isometric muscle contractions (14-17). A few decades later a force-velocity curve for concentric (41) and eccentric (55) contractions followed. These relationships were eventually understood when Huxley postulated his sliding filament theory (49), which includes the interaction of actin and myosin filaments. This commonly accepted theory describes the basic mechanics of a single fiber contraction in its longitudinal dimension.

Although experimental $(65,87,127)$ and numerical $(75,97,122,123)$ studies approach muscle behavior in one spatial dimension, multi-dimensional effects during contraction are essential for its mechanical functioning. Evidence exists for lateral force transmission between adjacent sarcomeres (76) and muscle fibers (100). Numerical studies have shown that geometrical effects are important to cardiac $(18,51)$ and skeletal muscle behavior $(33,108,109,116)$. Van Leeuwen et al (107) showed in twodimensional simulations that fibers diverge during contraction. They could solve the too low volume fraction by taking the third dimension into account. Only few experimental studies regard two- or three-dimensional muscle deformation. Combined measurements of length changes of superficial fibers, aponeuroses and total muscle length during contraction $(65,127)$ provide data related to two spatial dimensions. Zuurbier et al (125) concluded from such measurements that muscle deformation is essentially three-dimensional. Two-dimensional muscle surface strain distributions are

'In the remainder of this thesis, 'muscle' is used as a synonym for skeletal muscle tissue. Where smooth or cardiac muscle is meant, this will explicitly be mentioned. 
published for passive stretching (102) and only very recently for contractions (103). All aforementioned deformation measurements however, are based on two-dimensional views that can contain projection errors. One of the present objectives is to determine accurate surface strains during muscle contraction from three-dimensional deformation measurements.

The three-dimensional character of muscle mechanics is also reflected in the heterogeneity of intramuscular pressure distribution $(56,74,91)$. Sejersted (90) reviewed the literature regarding tissue pressure rise during contraction, mainly in relation to muscle perfusion. Muscle perfusion is impeded by tissue pressure rise, either as a result of tetanic contraction (8) or of artificial tissue pressure rise $(85,94)$. Current hypotheses regarding the interaction between perfusion and tissue pressure rise, which will be discussed later in this introduction (page 10), are merely based on experiments where tissue pressure is artificially controlled $(85,94)$. However, applied tissue pressure is homogeneous during these experiments, whereas it is distributed during contraction $(56,74,91)$. The spatial tissue pressure distribution in combination with the hierarchical organization of the vascular tree complicates the interaction between contraction and perfusion.

The vascular tree can be seen as a branching network of vessels, with one artery and one vein feeding and draining thousands of capillaries. Except for arteries, capillaries and veins, many other vessel types, each with a different function or constitution, are found in this network. A distinct hierarchical organization of the vascular tree exists, and the dimension and constitution of the vessels, and therefore also their mechanical behavior, strongly depends on hierarchical depth. Further, blood pressure or intravascular pressure decreases more than 10-fold in the hierarchical dimension, from more than $10 \mathrm{kPa}$ arterial pressure to less than $1 \mathrm{kPa}$ at the venous side. The organization of the vascular tree and the importance of. their constitution for the mechanical interaction between contraction and perfusion, will be discussed in more detail in the paragraph 'Perfusion' of this introduction (page 7).

Summarized, vessel wall properties and intravascular pressure relate to the hicrarchical dimension, whereas extravascular pressure depends on the spatial location because of the heterogeneous tissue pressure distribution. To explore this complex interaction between contraction and perfusion, experiments need support from numerical models and vice versa. On the one hand, models need verification and must be fine-tuned by experiments, while experiments can be performed to obtain proper model input. On the other hand, models can be used to predict, interpret or calculate phenomena that are impossible to measure experimentally. Such an 
interaction between experiments and simulations can only be successful performed in close harmony. Vankan et al (116) compared their model of perfused contracting skeletal muscle to perfusion experiments of Shrier and Magder (94). This comparison only involved specific conditions during which geometrical interactions are largely excluded. The present study aims at studying the spatial interaction between muscle perfusion and pressure development during contraction, using a close combination of experiments and numerical simulations with the aforementioned model of perfused contracting skeletal muscle (116).

To become familiar with mechanical muscle functioning and deformation in general, an introduction to muscle constitution and basic contraction mechanics is provided in the next paragraphs. This is followed by a description of current theories regarding muscle perfusion in relation to contraction. Further, the introduction provides an explanation of the use and constitution of the aforementioned numerical model of perfused skeletal muscle. Model parameters and equations will be placed in a physiological context. The description will not capture the exact mathematical implementation of perfusion in the model, as Vankan extensively discussed this in his thesis (111).

Finally, the main research objectives of the present study are being formulated and an outline of the thesis is provided.

\section{DEFORMATION}

\section{Muscle constitution and contraction}

Macroscopically, the main constituents of a skeletal muscle are the contractile muscle cells or myocytes, also referred to as muscle fibers, and the passive stiff non-linear elastic collagen tissues: aponeuroses and tendons. Aponeuroses or tendinous sheets are macroscopically organized as membranes to which muscle fibers attach. Aponeuroses and muscle fibers connect to bone directly or via tendons, bundled continuations of aponeuroses. Connective tissues run parallel to the active muscle components at each level of detail, as visualized in Figure 1.1. The epimysium or fascia, a strong connective tissue layer, completely surrounds the muscle, whereas perimysium separates bundles of fibers or fascicles and endomysium covers and electrically isolates individual fibers. Epiperi- and endomysium interconnect and continue in aponeuroses and tendons. They are important for serial as well as for lateral force transmission (100).

For some muscles, such as the anterior tibialis muscle, the fascia is a welldeveloped strong membrane that is firmly connected to the muscle and can 
hardly be dissected from the underlying tissue. To illustrate the strength and importance of the fascia to muscle functioning, the well-known compartment syndrome is a painful pathology in which the swollen anterior tibialis muscle is compressed in its fascia. It is treated by fascia cleavage. In other muscles such as the medial gastrocnemius muscle, the fascia is less developed.

Each myocyte contains hundreds of cylindrical myofibrils, highly arranged structures consisting of thousands of the fundamental contraction units named sarcomeres. Amongst a number of other molecules, they contain the most familiar muscle proteins actin and myosin. A myosin molecule with facing tails is accompanied by actin molecules at both sides. Actin is connected to the cytoskeleton, which provides the subcellular structure of the myocyte. Cross-bridges bind myosin heads with actin. For the highly arranged serial organization of sarcomeres, repeating dark and light bands can be distinguished in muscle cells giving skeletal muscle tissue its

Figure 1.1

Muscle gross structure. The interconnected epi-peri- and endomysium surround muscle, fascicles and fibers. Myocytes are built up of myofibrils, which contain the functional contraction units: sarcomeres. The most familiar muscle proteins actin and myosin, are constituents of the sarcomeres.

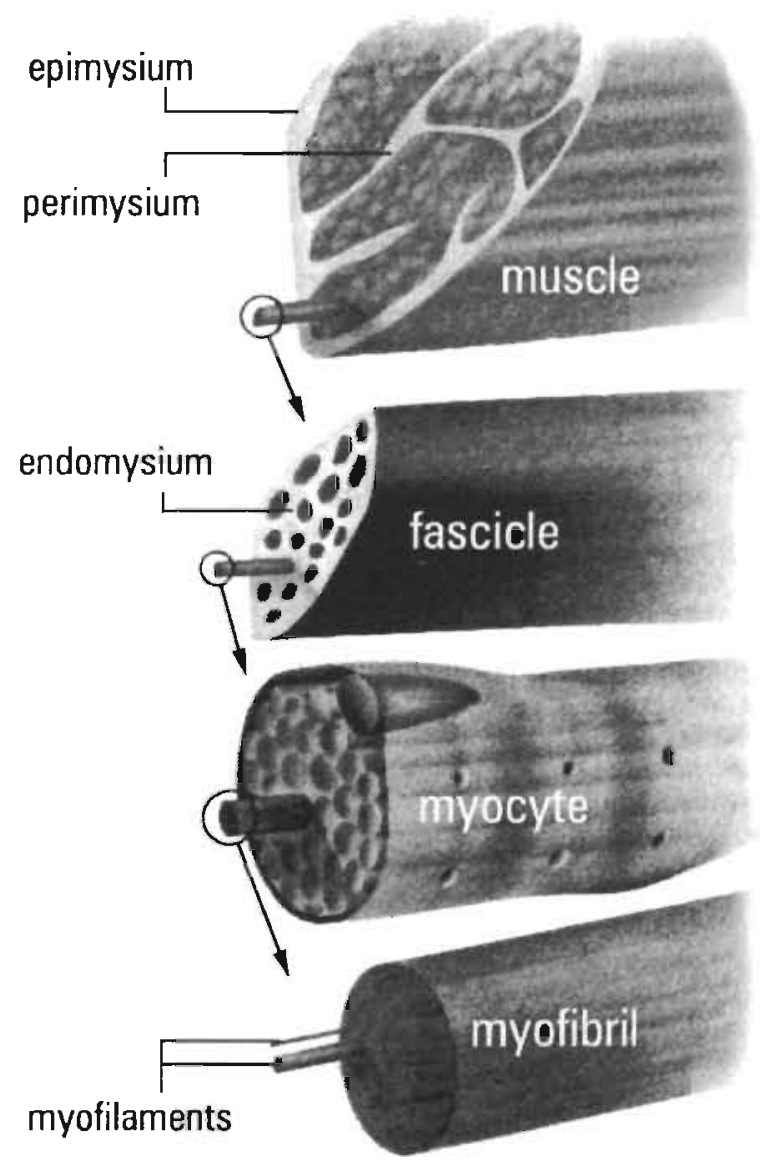


characteristic striation pattern. During contractions, active bending of the myosin heads results in sliding of the myosin and actin filaments alongside each other, thus shortening the sarcomere and consequently (part of) the muscle fiber. Sarcomere length reflects the overlap between actin and myosin, which relates to the number of cross-bridges and consequently to force. Sarcomeres function in between 1.5 and $3.5 \mu \mathrm{m}$, whereas their optimum length, i.e. the length at which maximum force can be generated, is approximately $2.1 \mu \mathrm{m}(35)$.

\section{Spatial interactions}

Sarcomeres are serially organized in myocytes, which are serially connected to aponeuroses and tendons. In a one-dimensional, single fiber approach, the passive tendon stretches and the active myocyte shortens during isometric contraction. Further, assuming isovolumic fiber deformation, the cell thickens. Thus, there is a close relationship between contraction, fiber deformation and consequently fiber force.

Muscle geometry (18) and the direct mechanical interaction between parallel fibers (100) disallow extrapolation of fiber mechanics to muscle mechanics. In addition, mechanical muscle functioning depends on a number of other variables. For instance, fibers within one muscle are not of the same length $(87,120)$, fiber type distribution is heterogeneous (3) and sarcomeres within a single fiber behave non-uniformly (54).

The relationship between geometrical effects and muscle functioning is reflected in muscle deformation. Because a tendon is stretched during an isometric muscle contraction, on the average muscle fibers shorten and thicken, assuming incompressibility of the tissue. Such deformation of central fibers will bulge the muscle and theoretically stretch peripheral cells. In turn, contraction of curved, peripheral fibers compresses the muscle contents, therewith increasing intramuscular pressure. The importance of these spatial interactions to muscle functioning depends on geometrical parameters like aponeurosis length, fiber curvature and pennation angle, rather than on muscle size (108).

Further, contractions will result in heterogeneous distributions of tissue pressure and other mechanical variables throughout the muscle. Tissue pressure during contraction gradually increases from zero at the muscle surface to high pressure in the muscle $(56,74)$. Sejersted et al (91) found a linear relationship between pressure and the distance from the muscle surface during isometric contraction of human skeletal muscle. The exact distribution of tissue pressure probably depends on muscle geometry and the properties of surrounding tissue. In contrast with the free muscle surface, intramuscular pressure below an aponeurosis can be any size, because a large pressure drop can exist over the thin aponeurosis from zero 
at the superficial side to intramuscular pressure at the muscular side. As a result of the tissue pressure distribution, myocytes experience different environmental mechanics, which influences their behavior. Regarding deformation, differences exist between superficial fibers, which can deform freely towards the superficial muscle side, and central fibers, completely surrounded by contracting tissue. This influences muscle deformation.

Deformation of the muscle surface and the aponeurosis during contraction has been studied extensively $(37,46,89,102,103,125)$, but there is no consensus regarding the strain distribution, especially with respect to the aponeurosis $(46,89,103,119,124)$. A common source of error is that all these studies performed two-dimensional deformation measurements of the curved muscle surface, which can lead to projection errors.

The three-dimensional character of muscle deformation is experimentally shown by Zuurbier and Huijing (125), who determined a decrease of the midsagittal section area of a medial gastrocnemius muscle during isometric contraction. This finding, which is also based on two-dimensional measurements, has important consequences for numerical models that address muscle deformation. These models are generally two-dimensional for the sake of simplicity. However, if deformation during contraction is essentially three-dimensional, these models should take the third dimension into account as well. This needs further exploration. A general objective of the present thesis is therefore to determine muscle deformation during contraction in three dimensions.

\section{PERFUSION}

\section{The vascular system}

Blood not only provides tissue with oxygen and other metabolites, but takes care of waste matter drainage as well. This is of major importance for proper functioning of all organs. Tissues can generally adapt local blood flow to what is needed. The most obvious example of flow adaptation is found in skeletal muscle perfusion, as during fast running, muscle blood flow increases up to 20-fold (2). Strikingly, muscle blood flow during tetanic contraction is impeded. Before explaining the interaction between contraction and perfusion in details, the following paragraphs will discuss regular organ perfusion.

The vascular system can be divided into a pulmonary and a systemic circulation, which are interconnected in series, both starting and ending in the heart. In the pulmonary circulation (from the right ventricle via the lungs and the left atrium to the left ventricle of the heart) blood becomes oxygenated, whereas in the systemic circulation (from the left ventricle via 
the organs and the right atrium back to the right ventricle of the heart), the oxygenated blood is passed to the organs that extract oxygen from the blood. All exchange of oxygen, metabolites and waste matter takes place in the 5-10 $\mu \mathrm{m}$ thick capillaries. Because outside the capillaries, transportation merely depends on diffusion, most living cells are located within a few microns of a capillary. Another reason for the presence of large numbers of capillaries is that all exchange between blood and tissue depends on capillary surface size available. In skeletal muscle for instance, more than 1000 capillaries exist per $\mathrm{mm}^{3}$ (21). This amount of capillaries indicates the complexity of the vascular tree; large numbers of branching vessels are needed in between the $\sim 30 \mathrm{~mm}$ thick aorta and the capillaries. The main function of large arteries is to transport blood from the heart to the organs. These arteries are generally named after their position in the body. The sural artery, for instance, feeds the three heads of the calf muscle, also named triceps surae muscles. Within organs, arteries and the smaller arterioles are usually named according to the grade of divergence. The arteries within an organ mainly transport and disperse the blood, whereas the arterioles not only disperse the blood, but also regulate local vessel resistance, and therewith local blood flow, by contraction of their muscular walls. Tissue auto-regulation, based on local chemical aspects, in combination with central control mechanisms determines the state of the arteriolar vascular walls. The most important in this respect are precapillary sphincters, located just in front of the capillaries.

After passing the capillaries, blood is collected in small venules, which converge into larger veins to eventually end up in the vena cava. Alongside an arteriole or artery, generally one or two similarly named venules or veins are found. The function of venules and small veins is to collect blood,

Figure 1.2

Mean blood pressure distribution in the hierarchical dimension of the vascular tree. Most of the pressure drop occurs in the capillaries.

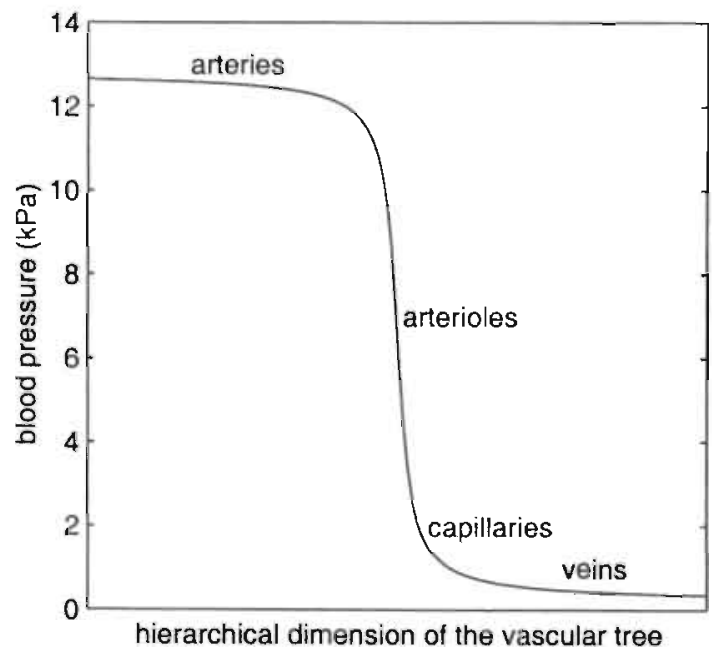


whereas larger veins also act as a reservoir of blood, which can be released by vessel constriction to enlarge the blood content in the arterial part of the system. Because of this function, veins generally have larger lumens than the arteries, and because of the relatively low venous blood pressure, they have thinner vessel walls.

To complete the anatomic description of the vascular system, anastomoses exist, which bypass part of the vascular tree. Those highly constrictable vessels for instance interconnect an arteriole and a venule, bypassing the capillary network in between. Such vessels are valuable to decrease resistance of an organ when the tissues metabolic activity is minor and the capillary system is largely closed by constriction of pre-capillary sphincters.

Together with the varying function of blood vessels along the vascular tree, the vascular wall composition changes. Furthermore, blood pressure decreases with hierarchical depth (Figure 1.2). Actually, arterial blood pressure is pulsating in between $11-16 \mathrm{kPa}$ as a result of the heartbeat, whereas pulsation attenuates towards the arterioles. To withstand the pulsating high arterial pressure, arteries have thick and compliant vessel walls and a large lumen (48). The walls of the smaller arterioles are stiffer and contain more muscular tissue, which enables adaptation of their lumen. Most of the decrease in blood pressure occurs in the arterioles. To allow exchange between blood and tissue at the level of capillaries, vessel walls are only one cell layer thick, whereas pressure is nearly constant $(\sim 3 \mathrm{kPa})$. Pressure in the returning part of the vascular system is in the order of $1-3 \mathrm{kPa}$. Therefore, vessel walls of venules and veins are much thinner and more flexible, compared to the arteries. Despite the thin wall, venules are relatively stiff because of their small lumens. To illustrate to difference in vessel wall properties, Figure 1.3 shows a histological slice of a cross-section through skeletal muscle tissue that contains cross-sections through an arteriole and a small vein close to each other.

Figure 1.3

Illustration of the differences between arteriolar and venous vessel wall properties

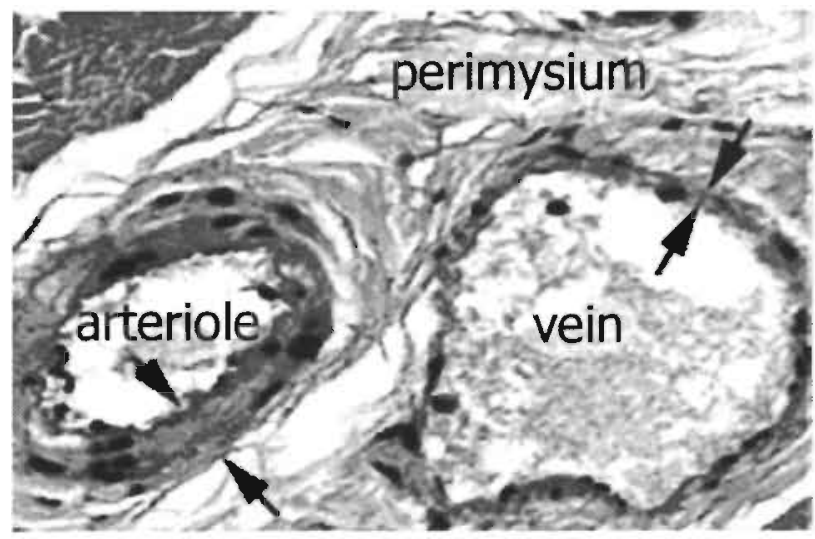




\section{Perfusion in contracting striated muscle}

Perfusion through a particular part of the vascular tree is determined by the pressure gradient and total resistance to flow of the fluid through the selected network of vessels. The geometry of the vascular tree, i.e. the parallel and serial organization and the diameters of all vessels, determines the resistance. Further, the diameter of an individual vessel is determined by its vascular wall properties and by the radially applied mechanical load, which equals the difference between intravascular and extravascular pressure and is referred to as transmural pressure. Extravascular pressure in striated muscle tissue is subject to rapid changes during contraction. Tissue pressure can rise up to more than arterial blood pressure during maximal contraction forces $(42,56,80,91)$. With constant vascular wall properties, either vascular resistance or intravascular pressure increases with increasing tissue pressure. This interaction between intramuscular pressure rise and perfusion makes striated muscle perfusion different from flow through regular organs, where rapid tissue pressure changes do not occur.

During repetitive contractions, venous outflow and therefore venous return to the heart is increased $(30,61,72)$. During sustained tetanic contraction however, decreased perfusion results $(8,90)$. Kirkebo and Wisnes $(56,121)$ showed similarities between the distributions of tissue pressure and intramuscular flow in successive experiments, which suggests spatial interaction between tissue pressure and perfusion.

Several theories are involved with the interaction between intramuscular pressure rise and perfusion. For instance, it is thought that pinching of larger vessels eliminates distal flow in the vascular tree (36). Currently the most supported hypothesis is the vascular waterfall theory. Permutt and Riley (78) were the first to mention the vascular waterfall, explained by pressurized collapsible tubes. They showed that the difference between inflow pressure and externally applied pressure on the tubes, and not between inflow pressure and outflow pressure, determines blood flow, provided that external pressure was in between inflow and outflow pressure. The independence of downstream pressure is referred to as vascular waterfall. Downey and Kirk (28) showed that the waterfall theory is also applicable to conditions, which include a range of external pressures.

Actually, the vascular waterfall theory predicts a local collapse of pressurized collapsible tubes, just in front of the outflow orifice. With respect to vascular trees, Mellander and Albert (66) argued that the distal resistance dramatically increases and that the mechanism should be referred to as a venous outflow orifice resistance, rather than a vascular or venous waterfall. The profit of this description is that it allows description of muscle perfusion during contraction by classic flow laws (6́6). 
The application of the vascular waterfall theory to the behavior of skeletal muscle perfusion during periods of raised tissue pressure is studied by means of airtight pressure boxes $(85,94)$. Increasing air pressure in the box artificially raises intramuscular pressure of muscles placed in these boxes. It is shown that blood flow is independent of downstream venous pressure, as long as the latter does not exceed box pressure (85), whereas blood flow decreases with increasing box pressure (94).

The advantage of the use of these pressure boxes is that high and steady pressures can be applied to a muscle for a longer period of time. It is questionable whether a steady state in perfusion is reached during a contraction. Further, unlike the distributed intramuscular pressure during actual muscle contraction $(56,74,91)$, a pressure box will affect tissue pressure to rise homogeneously. When the pressure is heterogeneously distributed, like during regular contractions, the interaction between tissue pressure and perfusion becomes more complicated. In that case, the distributed tissue pressure affects the spatial distribution of blood pressures in the muscle, on top of the ordinary hierarchical blood pressure distribution in the vascular system. This combination of spatial and hierarchical pressure gradients complicates the interaction between contraction.

The steady state situation in perfusion and the distributed intramuscular pressure during contraction are important discrepancies between pressure box experiments and contraction. Therefore, it is hypothesized that theories based on pressure box experiments cannot directly be extrapolated to explain the interaction between flow and tissue pressure during contraction. The interaction between contraction and muscle perfusion is further explored in this thesis. Both experiments and a numerical model will be invoked for this purpose. The model, which has proved to agree with pressure box results (116), is further explained in the following paragraphs.

\section{MODELING SKELETAL MUSCLE PERFUSION}

\section{Numerical models}

The present knowledge of muscle perfusion and mechanics is the result of both experimental and numerical research. Several numerical approaches are published. Many include tissue pressure building during skeletal muscle or left ventricular wall contractions without bothering blood flow $(33,75,109,122)$. Some stress the importance of muscle architecture for the mechanical behavior, i.e. deformation and tissue pressure development, during contractions $(18,33,107)$. Other models focus on perfusion of 
microvascular networks in general (83), or on muscle perfusion specifically (21). The latter lumped-parameter model supports the vascular waterfall theory, but cannot take spatial mechanical effects into account.

Several models include both contraction mechanics and perfusion. Arts (4) created a mathematical model that describes the interaction between left ventricular wall mechanics and tissue perfusion, whereas Vis (118) used the time-varying elastance concept of Krams $(57,58)$ to model the effect of tissue deformation and pressure on cardiac blood flow. Huyghe (50) tissue perfusion by describing it as fluid flow in a porous solid. Using a finite element formulation of this description, perfusion in contracting left ventricular wall tissue was studied. Based on this work of Huyghe (50), Vankan (111) implemented a description of contracting skeletal muscle perfusion in a finite element model. This particular model is used in the present study. The next paragraph will capture a description of the basic contents of the model, as far as these are necessary to understand the use of the model in the present study. For an extended description of the mathematical formulations is referred to the thesis of Vankan (111).

\section{The finite element model of perfused, contracting skeletal muscle}

The finite element method is a numerical tool in the field of continuum mechanics to calculate distributions of mechanical variables in a material under specific loading and boundary conditions. One of the basic assumptions in continuum mechanics is that properties of the concerned material are homogeneous and continuous at the considered level of detail. To calculate a particular lodding condition of a construction, a finite element model typically needs material properties and geometrical information of the construction, boundary conditions, external load, conservative laws regarding mass and momentum and constitutive equations that are to be solved. Geometrical information is provided in a finite element mesh, which is built up of voluminous elements, interconnected via nodes. Given the aforementioned model input, nodal displacements are calculated, and strain, stress or any other related mechanical variable can be determined per node in the mesh. In this way, spatial distributions for each desired parameter are obtained.

In the present finite element description of perfused skeletal muscle tissue, total mass is subdivided in a fluid mass (blood) and a solid mass (muscle tissue). Both are incompressible and masses cannot exchange. Further, solid mass is constant, whereas fluid volume can change because inflow not necessarily equals outflow of blood. Muscle mass is modeled as linear elastic material to which an additional stress component can be prescribed per element in a predefined direction, corresponding to the muscle fiber direction, to mimic muscle contraction $(20,114)$. 
The most important part of the model in the context of this thesis is the way fluid mass is modeled. The model does not contain a branching vascular tree as such, but blood flow through the muscle is considered as flow through a continuum of pores. The intramuscular vascular tree is classified in five hierarchical compartments, representing arteries, arterioles, capillaries, venules and veins. Each of these compartments is treated as a separate porous continuum with a certain blood volume. A piece of muscle tissue, the averaging volume, consists of a solid tissue mass and five blood volumes. These blood volumes are actually implemented as ratios of the total averaging volume, and are referred to as blood volume fraction or porosity. This way, a value for porosity per blood compartment per averaging volume in the tissue is obtained. It is assumed that the blood volume is equally distributed within the averaging volume. For statistical reasons, this assumption is valid as long as the considered volume is large with respect to the size of the vessels.

Hierarchical flow between adjacent blood compartments in an element is possible in both hierarchical directions, depending on the pressure gradient (white arrows in Figure 1.4). Further, spatial flow within one blood compartment can occur between neighboring elements. The spatial flow direction is indicated by black arrows in Figure 1.4 for the arterial and venous compartment only, because spatial flow mainly comes to the account of arteries and veins. The length of arterioles, venules and capillaries restrains substantial spatial flow in these three compartments.

Tissue perfusion in either the hierarchical or the spatial dimension is further determined by vascular resistance. This is defined by the

Figure 1.4

The organization of fluid mass and flow in the finite element model. The four striped areas on top of the figure represent four two-dimensional elements. The five boxes below represent the five blood compartments, each one with its own volume. In the hierarchical direction, adjacent compartments intercommunicate (white arrows). In the spatial direction, fluid flow between connected elements is possible (black arrows).

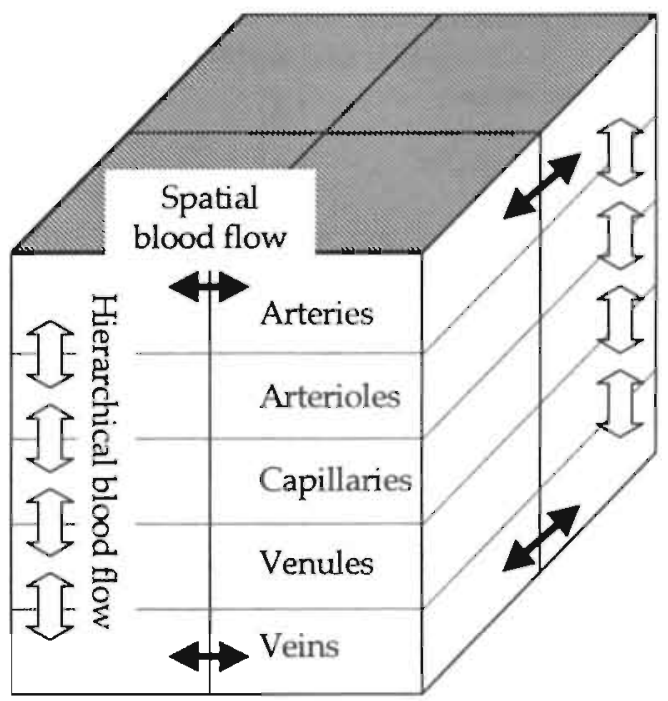


permeability of the tissue. In a three-dimensional situation with five blood compartments, spatial permeability in all directions for each compartment, and four permeabilities between adjacent compartments need to be determined for each element of the mesh. In all simulations in the present thesis, these are reduced to six permeabilities by assuming identical permeability in all spatial directions in arteries and veins, absent spatial permeability in the other blood compartments and a homogeneous spatial distribution of these variables throughout the muscle.

Permeability and porosity are dependent, as both parameters actually reflect different determinants of the same vessel diameters. The permeability $(\tilde{K})$ in blood compartment $(i)$ depends quadratically on the local porosity $\left(\widetilde{J}_{i}^{f}\right)$ via $\widetilde{K}_{i}=\widetilde{K}_{0_{i}}\left(\frac{\widetilde{n}^{f} i}{\widetilde{n}_{0}^{f}}\right)^{2}$, in which $\tilde{K}_{0_{i}}$ and $\widetilde{n}_{0}^{f}$, are reference values for permeability and blood volume fraction. Vessel diameters are affected by transmural pressure $\left(P_{T M}\right)$, which equals the difference between intravascular and extravascular or intramuscular pressure. The higher the intravascular relative to the extravascular pressure, the larger the vessel diameter and vice versa. The non-linear elasticity of the vessel wall is effectuated in the model by the arctangent function $\sqrt{n^{\prime}}=\sqrt{n_{0}}\left(1+\frac{2}{\pi} \arctan \left(\frac{P_{T M}-P_{s}}{P_{T M 0}}\right)\right)$, where $P_{s}$ and $P_{T M 0}$ are reference pressures (Figure 1.5). The maximum vessel volume equals $2 * \sqrt{n_{0}}{ }_{i}^{\prime}$.

\section{Figure 1.5}

The relationship between blood volume fraction and transmural pressure. in this example, $P_{s}=5 \mathrm{kPa}$ and $P_{\text {tm }}=3 \mathrm{kPa}$.

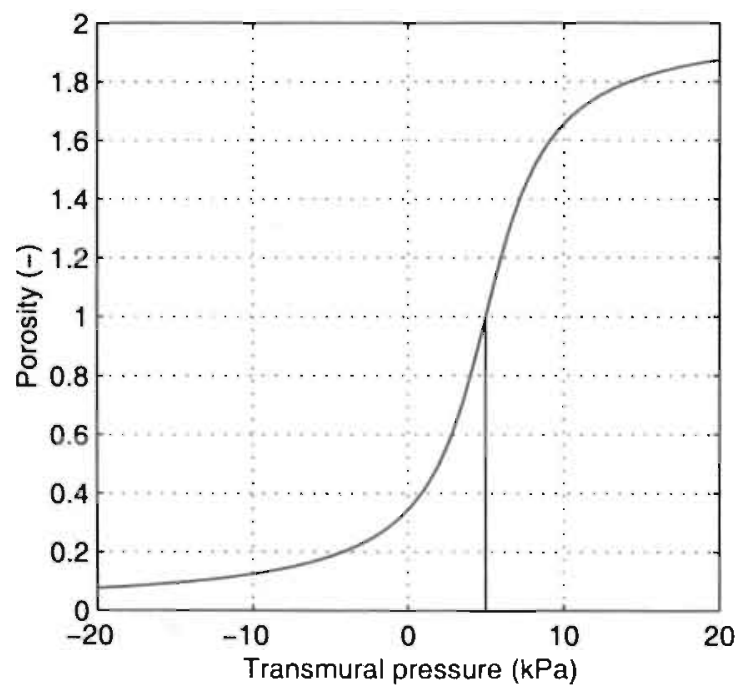




\section{Model parameters vs. physiology}

The aforementioned model variables and equations with respect to the implementation of perfusion can easily be identified as physiological or anatomical variables. For the implementation of blood perfusion, intramuscular pressure $\left(\mathrm{P}_{\mathrm{IM}}\right)$ and 6 blood pressures $\left(\mathrm{P}_{\mathrm{b}}\right)$ surrounding the 5 blood compartments are defined. These 7 pressures as well as blood volumes (porosity) per compartment and total vascular cross-sectional area of in a certain area (permeability) are all physiologically interpretable and principally measurable in experiments. This enables accurate comparison between experimental results and simulations. However, as all variables are spatially distributed throughout the muscle it is impossible to measure them simultaneously. Because geometrical information is basically incorporated in the finite element method, the model is a helpful tool in studying spatial distributions of the aforementioned variables, and therewith to study the interaction between contraction, deformation and perfusion. The model provides the opportunity to build an anatomically realistic two- or three-dimensional muscle geometry for which deformation, one of the basic output parameters, is calculated.

Furthermors, the relations that describe perfusion and the interaction between deformation and perfusion are also physiologically interpretable. During simulations, arterial and venous pressures are prescribed at the locations where an artery or vein is supposed to enter or leave the muscle. The arterial-venous pressure gradient drives hierarchical flow, whereas local pressure gradients within a blood compartment are responsible for spatial flow. During simulations of contraction, intramuscular pressure is calculated from tissue deformation. Tissue pressure in turn affects the vessel diameters via changes in transmural pressure. Consequently, blood volumes, vascular resistance and blood pressures change.

\section{OBJECTIVES AND OUTLINE}

The main objectives of the present study regard spatial effects of contractions. These are studied with respect to deformation and perfusion, as explained in detail in former paragraphs. These objectives are recalled as follows. The first one is to measure the three-dimensional deformation of skeletal muscle during contractions, and to determine the importance of taking the third dimension of deformation into account with respect to muscle functioning. The second aim is to study the interaction between contraction and perfusion, and to explore spatial effects that interfere with this interaction.

This thesis starts with the interaction between contraction and tissue perfusion. Results of total arterial and venous flow calculations with the 
finite element model of perfused contracting skeletal muscle are compared to total perfusion measurements of a rat medial gastrocnemius muscle. It is verified whether a vascular waterfall, which is inevitably present in highly controlled pressure box experiments, is effective during muscle contractions as well (chapter 2). The intramuscular, spatial interaction between the behavior of this vascular waterfall and the tissue pressure rise during contraction, which essentially differs from tissue pressure during pressure box experiments, is studied in chapter 3 .

Chapters 2 and 3 use a two-dimensional representation of the midsagittal section through the medial gastrocnemius muscle. In chapter 4 , the validity of such models to study mechanical muscle functioning is questioned. Muscle surface deformation during contraction is measured in three dimensions. Special attention is given to the differences in strain between aponeurosis tissue and muscle fibers and to the relationship between longitudinal and transverse strain during contraction.

To directly compare muscle surface deformation between model and experiment, geometrical model input must be realistic and anatomically correct. The fifth chapter aims at obtaining a detailed three-dimensional reconstruction of the medial gastrocnemius muscle, which is to be used in simulations. One of the major disadvantages of such a reconstruction regards the difficulties in obtaining accurate muscle fiber directions. It is thought that the diffusion weighted MRI technique can be used to obtain this information. The first objective of chapter 6 is to validate the method for this purpose, to obtain a realistic finite element mesh, including accurate muscle fiber directions, and to use it for calculation of muscle deformation during contraction.

The thesis ends with a general discussion, which recapitulates the present studies in general and points into directions for future research regarding spatial phenomena of skeletal muscle mechanics. 


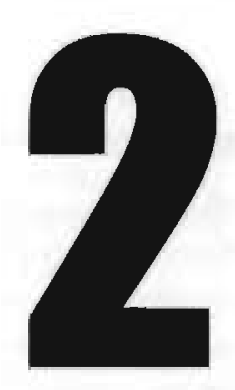

\section{THE EFFECT OF CONTRACTION ON TOTAL MUSCLE PERFUSION}

\footnotetext{
Total arterial and venous flow and volume change during isometric contractions of rat medial gastrocnemius muscle of different intensity are measured. Similar relationships between contraction and perfusion are calculated using the finite element model of perfused contracting skeletal muscle. Results are compared and the model is used to interpret experimental findings. These are discussed in the context of the vascular waterfall theory.
} 


\section{INTRODUCTION}

Skeletal muscle contraction has a major impact on muscle perfusion. During contraction blood is squeezed out of the muscle, whereas inflow is facilitated during relaxation $(30,61,72,92)$. This muscle pump function attributes to blood circulation during cyclic contractions. During sustained tetanic contraction however, the muscle pump is ineffective and perfusion remains impeded. Strong tissue pressure rise during contraction $(42,56,80$, 91 ) is held responsible. The literature regarding tissue pressure and muscle perfusion is reviewed by Sejersted and Hargens (90). Experiments during which intramuscular pressure was controlled by means of air-tight pressure boxes $(61,85)$, often in combination with artificial perfusion of the muscle $(22,94)$, show that muscle perfusion is independent of efferent blood pressure, as long as tissue pressure exceeds venous pressure $(85,94)$. It was concluded that the difference between arterial and tissue pressure, rather than the arterial-venous pressure gradient, determines muscle perfusion. This phenomenon is commonly accepted as the vascular waterfall theory $(12,22,28,85,93,94)$, referring to the pressure drop from intramuscular to systemic venous pressure near the outflow orifice and the independence of downstream pressure. Following the original findings of Permutt and Riley (78), who first demonstrated the mechanism using compressed collapsible tubes, some authors argue that the vascular waterfall is better named venous outflow orifice resistance, since collapse of distal veins initiates the characteristic venous pressure rise $(7,66)$.

The main advantage of the aforementioned pressure box experiments is that high and steady tissue pressures can be generated. However, the spatial intramuscular pressure distribution is different when comparing pressure box experiments (tissue pressure is homogeneous) with normal contractions (tissue pressure increases centripetally) $(56,74,91)$. Further, a steady state situation with respect to perfusion, which can be obtained during pressure box experiments, is not necessarily reached within the time period of a contraction. These two disagreements disallow direct extrapolation of pressure box experiments to actual contractions.

Two results of subsequent experiments of Wisnes and Kirkebo $(56,121)$ suggest that during a sustained contraction, flow distribution corresponds to tissue pressure distribution. To thoroughly examine this finding experimentally, local blood and tissue pressure must be determined throughout the muscle during tetanic contraction. Such experiments can hardly be performed for great difficulties of these measurements, in combination with the short time a forceful contraction can sustain.

Vankan et al recently used a finite element model of perfused contracting skeletal muscle to compute arterial and venous muscle flow (117) and 
intramuscular, capillary and venous pressure distribution (116) during isometric contractions. Actually, these simulations of contraction are merely used as an example of the model capabilities. The main aim of the former study was model verification by comparing its results with a lumped parameter model, whereas the latter one shows that model simulations of pressure box experiments agree with experimental findings of Shrier and Magder (94). From this comparison and the computations of blood pressure and flow during contraction it was concluded that the principles of the vascular waterfall theory apply to the model (116)

The finite element model calculates spatial blood pressure and flow distribution in five successive blood compartments, representing arteries, arterioles, capillaries, venules and veins. The interaction between the distributed tissue pressure and blood pressure is the result of compression of vessels, intermediated by vascular wall properties. For a comprehensive explanation of the model is referred to previous publications $(112,114,115)$. Because of the capabilities of the model to calculate the effect of contraction on the spatial distribution of intramuscular pressure, blood pressures and blood volumes in different blood compartments, it is a suitable tool to explore the vascular waterfall during contractions. However, the only direct comparison of computed flow during contraction with flow measurements regards the aforementioned comparison of an arterial and venous flow signal (117), which is merely published as an example and not discussed in detail. Before interpreting simulations of contraction and drawing physiologically relevant conclusions, a closer comparison of the model output with experimental data is desired. Detailed data describing the effect of a tetanic contraction on arterial or venous flow is not available in the literature.

The present study aims at describing the effect of an isometric, tetanic contraction on the total arterial and venous flow signals of a skeletal muscle during contractions of different intensity. The second goal is to perform a closer comparison between measured muscle perfusion during tetanic contraction and simulations with the aforementioned model. Further, using additional possibilities of the model, the present study verifies if the experimental results agree with the vascular waterfall theory. In this way, the hypothesis is studied that the vascular waterfall, which is inevitably present during pressure box experiments, is effective during contractions when tissue pressure is distributed. 


\section{MATERIAL AND METHODS}

\section{Preparation}

Experiments were performed in four male, 10-13 weeks old Lewis rats, anaesthetized with Sodium-Pentobarbital (Nembutal ${ }^{(-)}, 1.0 \mathrm{ml} / \mathrm{kg}$ body weight, i.p. injection) after short term $(<20 \mathrm{~s}) \mathrm{CO}_{2}$ sedation. SodiumPentobarbital was supplemented as necessary. During experiment and preparation, the rat was placed on a $38^{\circ} \mathrm{C}$ heating pad. Rectal temperature was controlled and held in between 36.5 and $37.5^{\circ} \mathrm{C}$ using an infrared lamp. The skin was removed from the left lower leg and upper leg muscles that attached to the tibia were dissected from their insertions to expose the sural artery and vein. These vessels and the sciatic nerve were carefully trimmed from their surroundings using a binocular microscope. The muscle was frequently moistened with saline $\left(37^{\circ} \mathrm{C}\right)$ to prevent the tissue from drying. Vessels that branched off distally and did not supply muscles of the triceps surae complex (TS) were cut between ligatures. The calcaneus bone was cut and the TS was dissected free from its surroundings, leaving the proximal origin intact. The soleus muscle was removed and vessels supplying ventral muscles were cut between ligatures. Knee ligaments were cut and the ventral part of the lower limb was removed to ensure that all blood passing the sural artery fed the TS only. Two pairs of tweezers, slid through the fascia lata, firmly attached the proximal and distal part of the femur to the lower traverse of a draw-bench (Zwick 1445). The calcaneus bone was fixed to the upper traverse. The muscle was loosely enwrapped with a piece of plastic foil, moistened with saline $\left(37^{\circ} \mathrm{C}\right)$, to prevent it from drying during the experiment.

A HSE stimulator was used to electrically stimulate the muscle via two electrode wires that were both gently wrapped around the sciatic nerve. Flow was measured using a transit time flow probe (Transonic " $0.5 \mathrm{~V}$ probe, Transonic Systems, Inc.), positioned around artery, vein, or both artery and vein at the same time. Space between probe and vessel was filled with electrode gel (Spectra ${ }^{\$ 60}$ ) to obtain an optimal flow signal. The builtin $100-\mathrm{Hz}$ low-pass filter of the Transonic ${ }^{\otimes}$ apparatus was used to filter the flow signal. Flow and force signals were acquired at $200 \mathrm{~Hz}$ with an Apple Macintosh 7200 PowerPC computer ${ }^{\text {( }}$, equipped with a 12 bits analogue-todigital conversion board (National Instruments ${ }^{6}$ ), programmed with Labview 3.1 (Labview, Inc.). The flow probe was calibrated at zero flow and at $2.5 \mathrm{ml} \cdot \mathrm{min}^{-1}$ prior to each experiment, following the description of the manual (1). Flows were calculated in $\mathrm{ml} \cdot \mathrm{min}^{-1} \cdot 100 \mathrm{~g}^{-1}$, whereas axial force was transposed into fiber tension $(\mathrm{kPa})$, using muscle fiber length of the medial gastrocnemius muscle (GCM) and total TS weight. 
Arterial and venous flow signals were measured separately, to determine the mechanical effect of a contraction on these signals, respectively. Limited space around the vessels did not allow simultaneous measurement of arterial and venous flow during one contraction. Therefore, measurements were successively performed for each animal. Total muscle volume change $\left(\mathrm{dV}_{\mathrm{IM}}\right)$ was measured in one experiment as the difference between in- and outflow, by placing the probe around the artery and vein together. In a steady state situation, simultaneous measurement of afferent and efferent flow resulted in a pulsating signal in which mean flow level was zero. During contractions, this signal immediately showed $d V_{I M}$.

\section{Protocol}

First, optimal muscle length $\left(l_{0}\right)$, yielding maximal isometric force, was determined by measuring force during short $(200 \mathrm{~ms})$ tetanic contractions with submaximum force (stimulation: $0.4 \mathrm{~V}, 100 \mathrm{~Hz}$ ) at different lengths. Starting at the muscle length at which passive force equaled $0.2 \mathrm{~N}$, i.e. just below optimal length, contractions were separated by a 20 seconds resting period, during which muscle length was increased by approximately $1 \mathrm{~mm}$. This procedure continued until the muscle was $3 \mathrm{~mm}$ longer than $l_{0}$. The muscle was then set to $l_{0}$ and muscle fiber length was measured. After the experiment, the muscle was weighted. At optimal length the maximal stimulation voltage $\left(V_{M}\right)$ was determined by increasing voltage by $0.1 \mathrm{~V}$ at $100 \mathrm{~Hz}$ until maximal contraction force was reached. $V_{\mathrm{h}}$ was typically in between 0.6 and $0.9 \mathrm{~V}$.

The stimulation protocol during the experiments was as follows. First, 10 unrecorded contractions of $800 \mathrm{~ms}$ at $100 \mathrm{~Hz}, \mathrm{~V}_{\mathrm{M}}$, were performed, with a resting period of $9.2 \mathrm{~s}$ in between the contractions. As a result, blood flow increased from resting level $\left(\sim 3.5 \mathrm{ml} \cdot \mathrm{min}^{-1} \cdot 100 \mathrm{~g}^{-1}\right)$ to approximately $20 \mathrm{ml} \cdot \mathrm{min}^{-1} \cdot 100 \mathrm{~g}^{-1}$. More contractions at this intensity would not further increase muscle blood flow. This particular flow level will be referred to as $Q_{0}$. After these 10 contractions, recording of flow and force started. Using the same time-sequence, the muscle was alternately stimulated at $100 \mathrm{~Hz}$, $\mathrm{V}_{\mathrm{M}}$ to produce maximal force, and at a lower level for submaximum forces. The high contraction forces were necessary to keep the flow level constant at $Q_{\alpha}$ and were excluded in the post-processing. The submaximum level was imposed by either lowering the stimulation frequency (to a value in between 30 and $100 \mathrm{~Hz}$ ) or stimulation voltage (in between $0.05 \mathrm{~V}$ and $\mathrm{V}_{\mathrm{M}}$ ). In between series of 20 to 40 contractions, the muscle was given a 5-minutes resting period. 


\section{Post-processing}

Mean arterial flow levels during the last second before a contraction $\left(Q_{0}\right)$, and during the last $500 \mathrm{~ms}$ of each $800 \mathrm{~ms}$ contraction, were calculated. Mean systolic and diastolic flow levels were calculated from the arterial signals, being the means of maximum and minimum flow levels in each heart beat cycle in the mentioned time periods, respectively. Further, systolic, diastolic and mean flow ratio's $\left(R_{0}, R_{0 D}, R_{O}\right.$, respectively) were determined for each contraction by dividing the three aforementioned mean flow levels during contraction by $Q_{0}$. Unlike absolute flow levels, these ratios can be compared between different animals. For measurements of $\mathrm{dV}_{\mathrm{IM}}$ the arterial-venous measurements were used. Volume changes were calculated into $\%$ of total muscle weight.

\section{Simulations}

Numerical simulations of skeletal muscle perfusion were performed with the finite element model that has previously been described in the literature $(112,114,115)$. Muscle geometry was deviated from a longitudinal section of rat GCM. The mesh and parameters for perfusion and contraction were identical to those Vankan et al used to compare arterial and venous signals between simulations and experiments (117). Arterial pressure was prescribed at $10 \mathrm{kPa}$, venous pressure at $\delta .5 \mathrm{kPa}$. The arterial, venous and arterial-venous flow signals were computed for an 800 -ms contraction with a 100-ms time-to-peak-force and relaxation time. These signals were used for comparison with raw experimental data.

A simulation in which active contraction stress linearly increased from 0 to $130 \mathrm{kPa}$ in 10 seconds provided the relationship between muscle tension and flow ratio, as if it were obtained by a number of separate contractions. This was compared to the experimental relationship between muscle tension and $\mathrm{R}_{\mathrm{Q}}$.

\section{RESULTS}

During a total number of 710 contractions, which excludes the alternating contractions at maximal stimulation intensity, force and accompanying flow were recorded. Of these recordings, 547 contained arterial, 110 venous and 53 arterial-venous flow data. In Figure 2.1, Figure 2.2 and Figure 2.4, solid lines represent experimental data, and dotted lines are results of finite element simulations. 


\section{Dynamic flow response}

An example of the effect of a contraction at the arterial flow is presented in Figure 2.1. Figure 2.1A shows measured (solid line) and simulated (dotted line) force signals. The heartbeat can easily be identified in the arterial signal and a periodical variation of circa $1 \mathrm{~s}$ as a result of the breathing

\section{Figure 2.1}

Example of a simultaneous registration (solid lines) of muscle tension ( $A$ ) and arterial flow' (B). Active muscle rension, prescribed to the finite element simulation and the calculated arterial flow are added to these graphs (dotted lines).

\section{Figure 2.2}

Example of a simultaneous registration (solid lines) of muscle tension (A) and venous flow ( $B$, enlarged in $C$ ). Active muscle tension, prescribed to the finite element simulation and the calculated venous flow are added to these graphs (dotted lines).
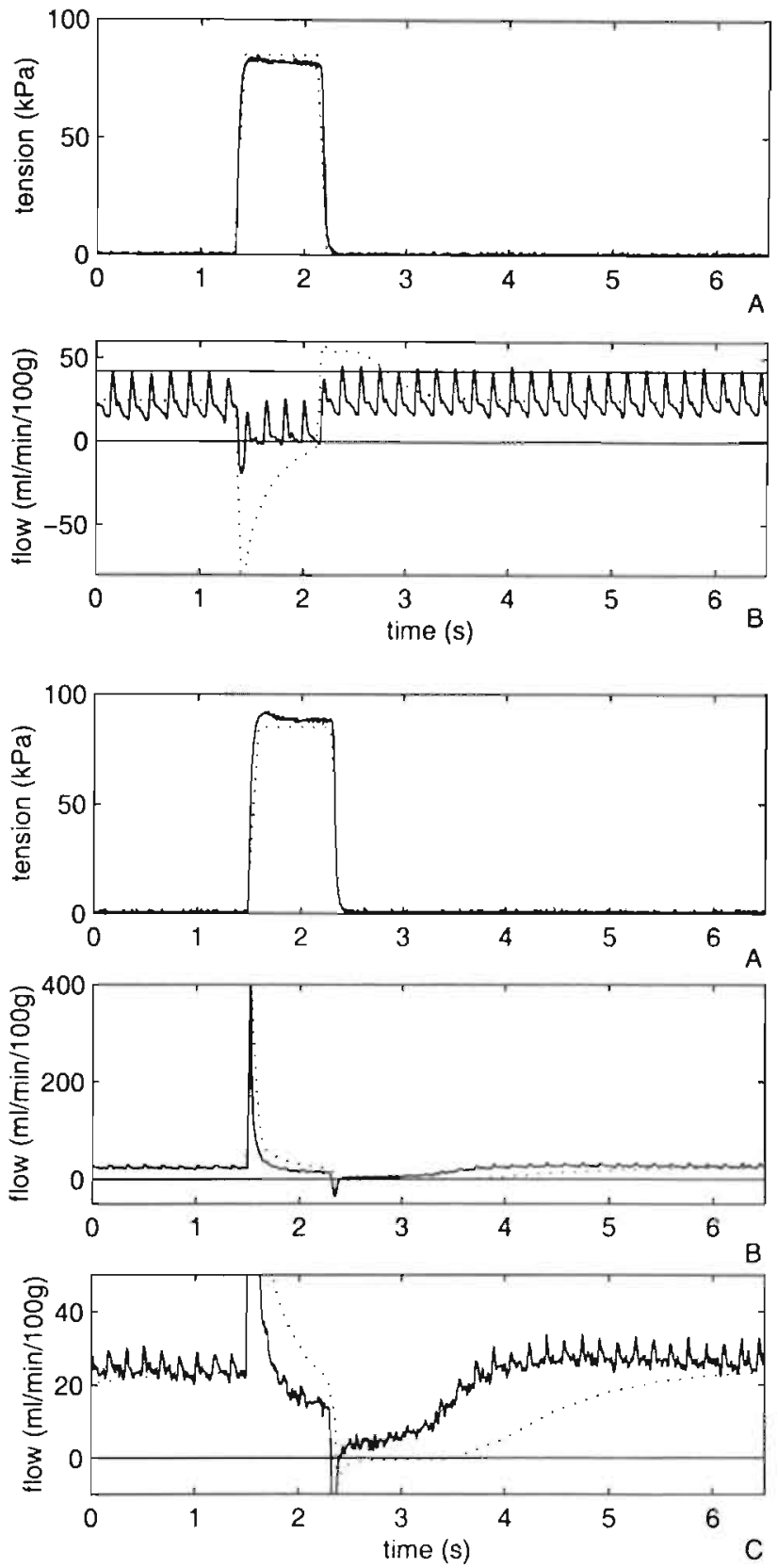
cycle is visible (solid line in Figure 2.1B). During contraction arterial flow initially changes direction, which means that a quick outflow occurs. Within $150 \mathrm{~ms}$, flow stabilizes at a steady level, depending on contraction force. During relaxation, flow immediately increases to a level that is 5 to $20 \%$ higher than $Q_{0}$. It takes 2 to 3 seconds before the signal is returned to the original resting level.

Essential characteristics like the initial quick outflow peak and the period of increased inflow after contraction are present in the calculated arterial flow signal as well (dotted line, Figure 2.1B). Simulated flow decrease during contraction and increase during relaxation are large relative to the experimental result, whereas the steady state arterial flow level during contraction is hardly reached in the simulation.

In $\sim 40 \%$ of the measurements, the heartbeat could be identified in the venous signal. An example of a venous flow registration (Figure 2.2B, C) with accompanying force signal is plotted in Figure 2.2. The level of the huge outflow peak that is seen at the start of the contraction equals 16 times $Q_{0}$ in this example, whereas it maximally reaches 20 times $Q_{0}$ at the maximum active muscle tension of $120 \mathrm{kPa}$. This peak declines much slower than the arterial outflow peak, theoretically ending up at the same

\section{Figure 2.3}

Example of a simultaneous registration (solid lines) of muscle tension (A) and arterial minus venous flow (B). The cumulative sum of arterial minus venous fiow ( $C$ ) equals the actual vascular volume change during the contraction as a percentage of initial vascular volume. The right column $(D$, E, F) contains simulation results. Note that the vertical axes of graphs $B$ and $E$ and $C$ and $F$ are different.
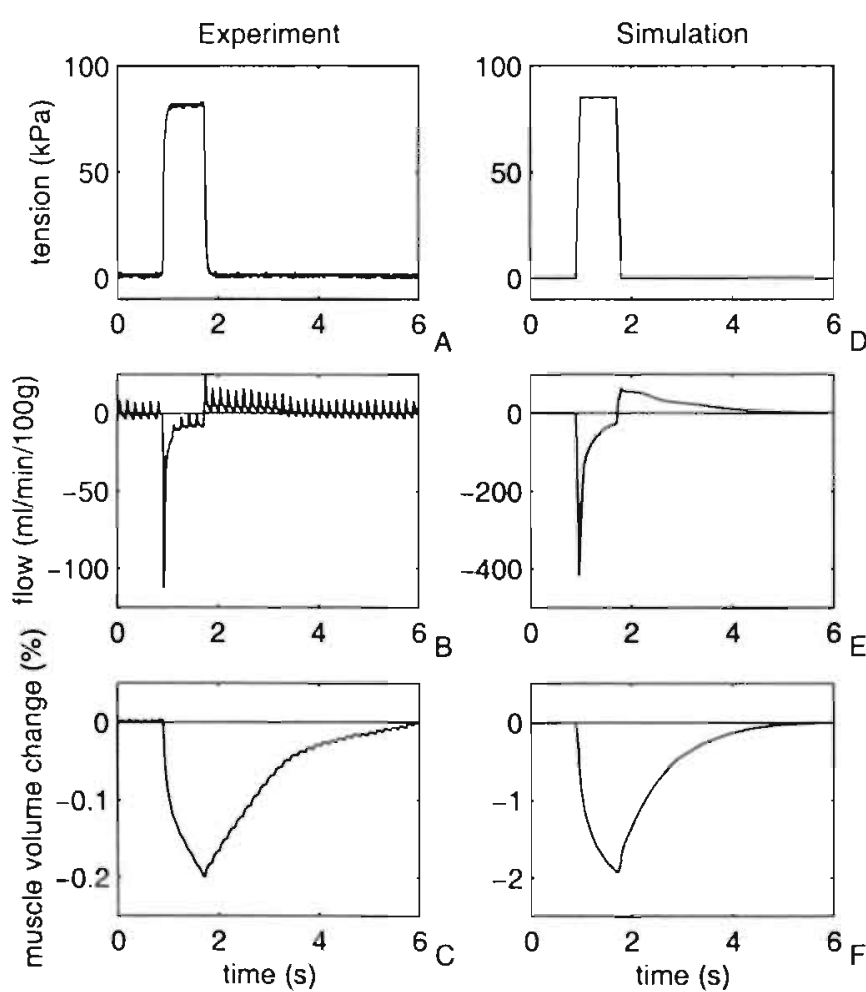
level as the arterial inflow during contraction does (Figure 2.1B). However, after $800 \mathrm{~ms}$ of contraction a steady state is not yet reached. During relaxation, a short period of venous inflow is followed by a characteristic period of very low flow. Approximately $1.0 \mathrm{~s}$ after the contraction stopped, flow increases to $Q_{0}$ in $0.5 \mathrm{~s}$.

In the simulation, the quick outflow peak is of similar height as in experiments, and a steady state is not reached. The outflow peak declines slower than in experiments. During relaxation, the characteristic negative flow peak precedes a 1-s period of very little venous outflow, similar to the experimental data, but it takes more time to return to $Q_{0}$.

A sample tracing of the arterial-venous signal is shown in Figure 2.3B, the cumulative signal is plotted in Figure 2.3C and force is shown in Figure 2.3C. The quick volume change during the first part of the contraction is followed by a slower decline during the rest of the contraction (Figure 2.3B, C). At the end of the contraction, muscle volume decreases $\sim 0.2 \%$ in this example.

The volume change in the simulation is shown in Figure 2.3E and $F$. The shapes of the curves are very similar between simulations and experiments, but quantitatively, volume change during contraction in the simulation is approximately ten times the experimental volume change. Note that the vertical axes of Figure $2.3 \mathrm{~B}$ and $\mathrm{E}$, and $\mathrm{C}$ and $\mathrm{F}$ are different.

\begin{abstract}
Figure 2.4
Mean flow ratio of 547 contractions, obtained from 4 experiments, is plotted against active muscle tension ( $\sigma$ ). The regression line equation of the leastsquares fit through the experimental data (solid line) is given in Table 2.1. The dotted line represents the result of a finite element simulation in which muscle tension linearly increased from 0 to $130 \mathrm{kPa}$ in 10 seconds.
\end{abstract}

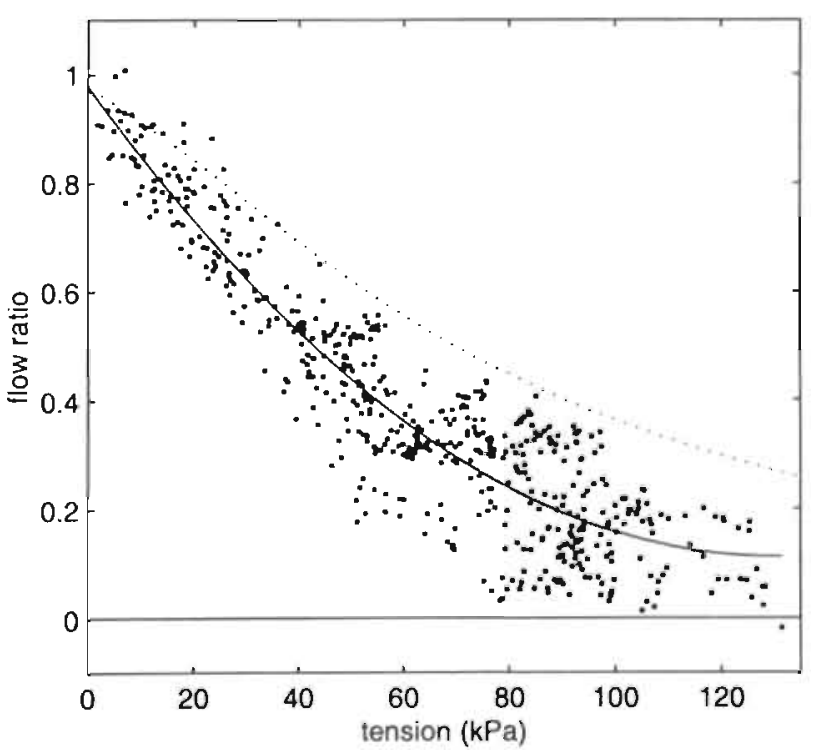


Chapter 2: The effect of contraction on total muscle perfusion

\section{Steady state flow}

Flow ratios, calculated from the steady state in the arterial signal, are shown as a function of muscle tension $(\sigma)$ in Figure 2.4. Using a leastsquares fit, a second-order curve was fitted through these data (Table 2.1). The curve levels off at $R_{Q}=0.1$. In the simulation, flow ratio also declines with increasing tension, following a similar kind of curve. However, the impact of a contraction on flow ratio is larger in experiments than in simulations, while the simulation relationship levels off at higher tensions than the fitted curve through the experimental data.

Figure 2.5 shows the systolic and diastolic flow ratio with accompanying regression lines. Because a heartbeat is not included in model simulations,

\section{Table 2.1}

The relationships of mean $\left(R_{Q}\right)$ systolic ( $\left.R_{Q}\right)$ and diastolic $\left(R_{Q}\right)$ flow ratio with active muscle tension $(\sigma)$, derived from a least squares fit on the experimental data. Regression line equations are valid in the range of 0 to $135 \mathrm{kPa}$ tension. r: correlation coefficient.

\begin{tabular}{l|ll}
\hline Flow ratio & Regression line equation $(\sigma<135 \mathrm{kPa})$ & $\mathrm{r}$ \\
\hline Mean flow & $\mathrm{R}_{\mathrm{Q}}=5.12^{\star} 10^{-5 *} \sigma^{2}-1.33^{*} 10^{-2 *} \sigma+0.98$ & 0.93 \\
Systolic flow & $\mathrm{R}_{\mathrm{O}}=5.65^{\star} 10^{-5 *} \sigma^{2}-1.42^{*} 10^{-2 *} \sigma+1.64$ & 0.80 \\
Diastolic flow & $\mathrm{R}_{\mathrm{Qd}}=3.53^{\star} 10^{-5 *} \sigma^{2}-1.10^{\star} 10^{-2} \sigma+0.65$ & 0.89 \\
\hline
\end{tabular}

\section{Figure 2.5}

Systolic and diastolic flow ratio, calculated as a ratio of mean total blood flow from all contractions presented in Figure 2.4. The regression line equations are given in Table 2.1.

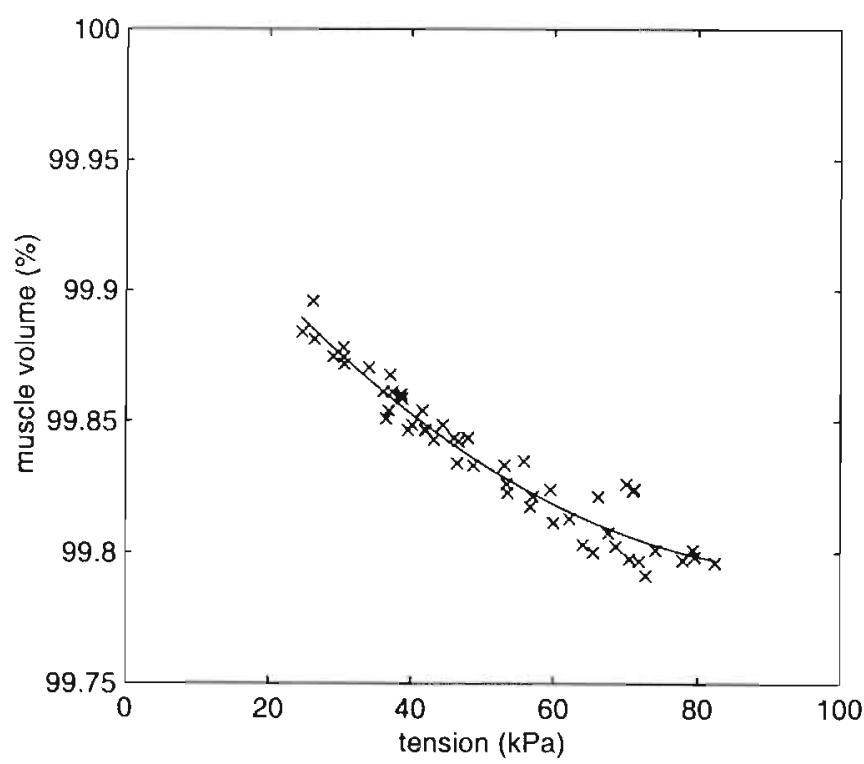


only experimental data are shown. Regression line equations of mean, systolic and diastolic flow ratios are provided in Table 2.1.

Finally, maximal $\mathrm{dV}_{\mathbb{I M}}$, measured after 800 -ms contraction is plotted against muscle tension in Figure 2.6. The regression line between active tension and $\mathrm{dV}_{\mathrm{IM}}$ is based on 54 measurements (Figure 2.6), and the correlation between the variables is high $(\mathrm{r}=0.97)$. Maximal $\mathrm{dV}_{\mathrm{IM}}$ reaches $0.21 \%$ of total muscle weight at $80 \mathrm{kPa}$ muscle tension.

\section{DISCUSSION}

\section{Experimental results}

The impact of an 800-ms tetanic contraction on arterial and venous flow was measured. Two representative examples of these tracks (Figure 2.1 and Figure 2.2) during contraction are plotted. The effect of contraction on mean flow ratio clearly depends on active muscle tension; mean flow ratio levels off at 0.1 during forceful contractions (Figure 2.4 and Table 2.1). $\mathrm{R}_{\mathrm{Os}}(\mathrm{r}=0.80$ ) and $R_{x+4}(r=0.89)$ also correlate to active muscle tension (Figure 2.5$)$. There is a constant difference between the functions that describe the relation between active tension and $R_{\mathrm{c}_{\mathrm{s}}}$ and $\mathrm{R}_{\mathrm{Od}}$, respectively; contraction results in translation of the pulsating arterial signal, rather than in a gradually decrease or increase of pulsation.

\section{Figure 2.6}

Muscle volume as a percentage of total initial muscle volume in the TS. The results of 2 experiments $(53$ contractions) are plotted against muscle tension. Regression line equation equals

$R_{Q}=1.82 * 10^{5 *} \sigma^{2}-3.54 * 10^{3 *} \sigma+99.97$, $r=0.97$. Maximum measured muscle volume change is $0.21 \%$ at $83 \mathrm{kPa}$ muscle tension.

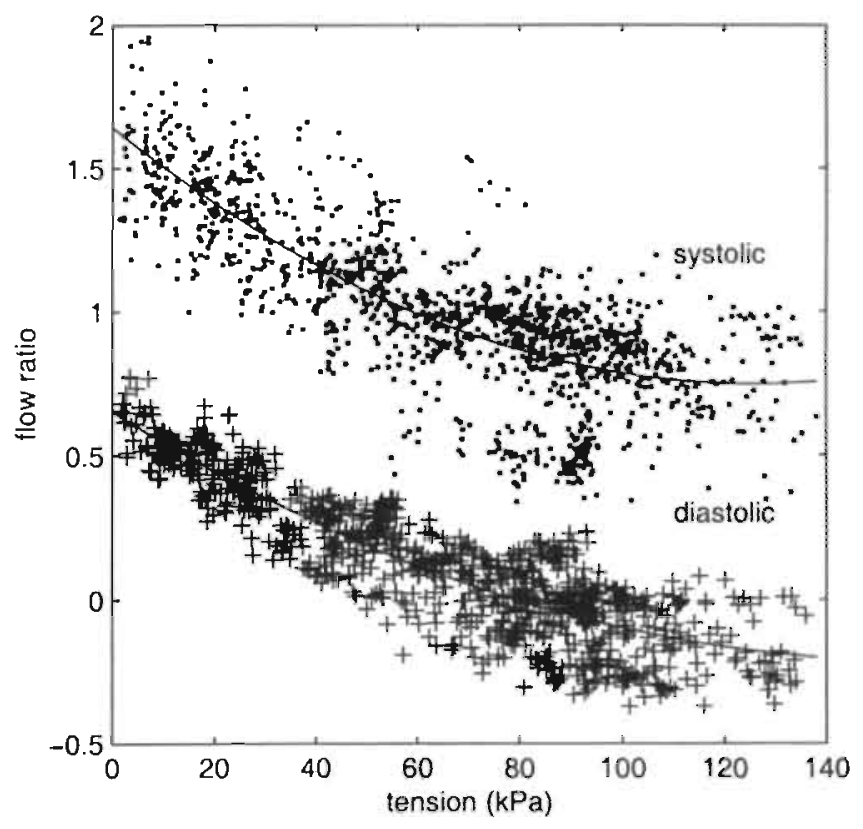


The short period of increased arterial blood flow during relaxation is variable in height and length in experiments. In simulations, it is evidently present (Figure 2.1). A likely physiological explanation for these patterns is involved with elastic recoil of emptied vessels, which generates low intraluminal pressures during relaxation (92). This explains the increased arterial inflow as well as the characteristic, relatively long period $(\sim 1$ second in the example of Figure 2.2) of low venous outflow. Arterial flow fills the emptied part of the vasculature, whereas venous outflow only reoccurs when vessels are refilled.

Volume change during contractions is small. At higher muscle tension, maximal volume change tends to level off at only $0.21 \%$ of muscle volume, which corresponds to $6.3^{\star} 10^{-3} \mathrm{ml}$ blood for the $\sim 3$-grams weighting rat gastrocnemius muscle. From Figure 2.3, it can be seen that during contraction, a quick volume change is followed by a period of slower muscle volume decrease. The first phase takes $\sim 150 \mathrm{~ms}$, and is the result of blood that is squeezed out at the arterial and especially at the venous side (Figure $2.1 \mathrm{~B}$ and Figure 2.2B). During the second phase, venous outflow slowly decreases, and there is limited inflow from the arterial side. Assumed that intramuscular blood volume is $8 \%$ of total muscle volume (21), the maximum muscle volume change of $0.21 \%$ equals $2.6 \%$ of the vascular volume. Note that $\sim 8 \%$ blood volume relates to maximally vasodilated muscle; the changed blood volume fraction may be somewhat more than $2.6 \%$ during these experiments. Further, the presented volume changes relate to 800 -ms contractions. In this time-period, a steady state situation is not reached, and $\mathrm{dV}_{\mathrm{IM}}$ increases with contraction time (Figure 2.3). In this respect, 800-ms contractions strongly differ from pressure box experiments, in which measurements are performed in a steady state situation.

Judging from the height of the arterial (Figure 2.1) and venous (Figure 2.2) flow peaks during contraction, most of the vascular volume change occurs at the venous side. Braakman et al (21) collected data of skeletal muscle vascular trees from the literature and divided the vascular system into 17 compartments. According to their findings, blood volume in the last venous compartment, the $2^{\text {nd }}$ order draining veins, is $2.4 \%$ of the vascular volume, whereas that in the $3^{\text {rd }}$ order draining veins is $10.9 \%$ and smaller vessels contain an even larger fraction of total muscle blood volume. Since $\mathrm{dV}_{\mathrm{IM}}$ during strong contractions is only $2.6 \%$ of total intramuscular blood volume, whereas $\mathrm{R}_{\mathrm{Q}}$ is $0.1 \%$, it is hypothesized that volume decrease is mainly located in the distal intramuscular venous compartment. If smaller vessels were emptied, slight diameter changes would have resulted in larger $\mathrm{dV}_{\mathrm{IM}}$. 
Even during forceful tetanic contractions of $120 \mathrm{kPa}$, muscle perfusion is not completely arrested. The relationship between flow ratio and muscle tension levels off at $\sim 10 \% Q_{0}$ (Figure 2.4). Because branching trees are cut and surrounding tissues are completely removed, this cannot be the result of extramuscular circulation. From the separate diastolic and systolic flow ratios, it can be seen that at tensions larger than $80 \mathrm{kPa}$, average diastolic flow is inverted. During systole however, flow ratio never decreases below 0.4 , whereas average $R_{Q s}$ levels off at 0.7 .

\section{FEM results}

Qualitatively the arterial, venous and arterial-venous flow curves, calculated by the finite element model, are similar to the experimental ones. Quantitatively however, the effect of contraction on perfusion is generally larger in simulations than in experiments. The simulated arterial outflow peak is higher than the experimental peak, whereas the decline is slower. As a result, in the presented simulation, arterial flow remains negative during the $800-\mathrm{ms}$ contraction, whereas, unlike in the experiments, a steady state inflow is not yet reached (Figure 2.1). During relaxation, arterial inflow increases for a second, before returning to $Q_{0}$. In experiments, this period of increased inflow is extended, but less pronounced.

The venous flow peak of the simulation compares closer to the experiments than the arterial curve does. Peak height is almost similar (Figure 2.2B), but the simulation curve declines slower (Figure 2.2C). The characteristic negative outflow peak during the relaxation and the period of very low outflow afterwards are present in both simulation and experiment. Although returning to the original flow level takes longer than during experiments, the similarity between model and experiment is obvious.

As expected from the separate arterial and venous signals, muscle volume change during contraction is larger in simulations than in experiments. Yet, the shape of the curves is very similar, and the initial quick volume change is clearly followed by a slower volume decrease as mentioned for the experimental data. Like in the experiments, volume still decrcasis after $800 \mathrm{~ms}$ of contraction.

The FE model allows closer interpretation of the blood volume change. Figure 2.7 shows the change in total muscle volume: as a percentage of initial muscle volume (Figure 2.7A equals Figure 2.3F) and a subdivision of this gross effect into changes in each of the five blood compartments (Figure 2.7B). The initial quick volume change comes to the account of the venous compartment, which does not change volume during the r'mainde'r of the contraction. The second, slower part of the total volume change during contraction, is merely the result of arterial volume change, but other 
compartments also slightly change volume during the entire contraction period.

The initial effect of contraction on the venous compartment, which is only followed by slower volume decreases in other blood compartments when contraction sustains, enforces the hypothesis that veins are the most important vessels with respect to the interaction between contraction and perfusion. This agrees to the vascular waterfall theory, as well as to the description of the venous outflow orifice resistance by Badeer and Hicks (7). In Figure 2.7B, a further decrease in venous volume after muscle relaxation is seen. This can be explained from elastic recoil of small vessels, which is previously mentioned to explain the period of low venous outflow. If due to elastic recoil in venules and capillaries, local blood pressure decreases below venous pressure, these compartments may be filled from the venous side.

The quantitative differences in volume change, arterial and venous flow signals between experiments and simulation, can be explained in several ways. For instance, input parameters of the separate compartments in the model may need adaptation regarding permeability, porosity or the relationship between blood volume fraction and transmural pressure. However, most parameters are fair estimates, obtained from the literature, and there are no arguments for varying particular parameters in a specific way. Permeability and porosity of the blood compartments, obtained from data collected by Braakman et al (21), are based on vasodilated muscle. This

\section{Figure 2.7}

Total muscle volume change as a percentage of initial muscle volume (A) calculated by the finite element model and the volume changes (\% of total initial muscle volume) of the five separate blood compartments (B) are plotted. Note that the venous compartment is the first one being emptied during contraction, followed by the other compartments. The venous and arterial compartments account for most of the volume change.
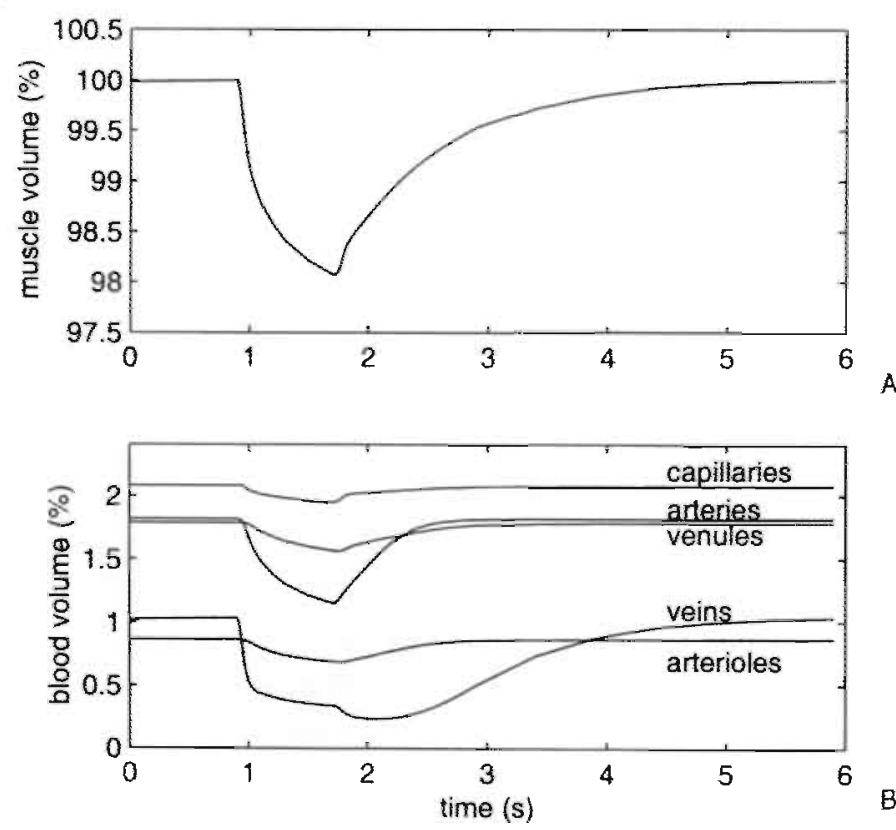
may cause an overestimation of blood volume in the model, but it will not explain all of the quantitative differences. Further, reference output like hierarchical pressure gradients, agree to physiological data (Figure 2.8). This suggests that hierarchical model input is adequate. It is thought that other aspects of the model input may need recapitulation. Two of these are discussed below.

First, because most of the excessive volume change comes to the account of venous blood, decreasing the venous compartment volume reduces outflow volume. Actually, the volume relates to the compartmentalization of the vascular tree in only five compartments, which is a rough approach of its complex hierarchical structure. A possible solution is to increase the number of compartments in future studies. Such extension would however increase the number of variables and to which proper settings are not currently available. Besides, each extra available blood compartment would increase computation time.

The second consideration regarding the presently used parameter input of the vascular tree regards the assumed continuum of vessels and the homogeneity of perfusion parameters in all spatial directions. For instance, the orientation of capillaries is predominantly parallel to the muscle fiber direction. Spatial permeability can be adjusted accordingly. More important for the large blood volume change during contraction is the assumed continuum regarding arteries and veins. Especially the 1st and 2nd order draining veins and feeding arteries are sparsely present. The pathway for blood from a particular capillary to the outflow orifice is via the venules and veins in between. For the assumption of continuity and homogeneity of all vessels, spatial fluid flow in the model is possible in a straight line from

Figure 2.8

Hierarchical pressure distribution in a central node of the finite element model at rest. Calculated pressures in the different blood compartments correspond to physiological pressures in arteries, arterioles, capillaries, venules and veins.

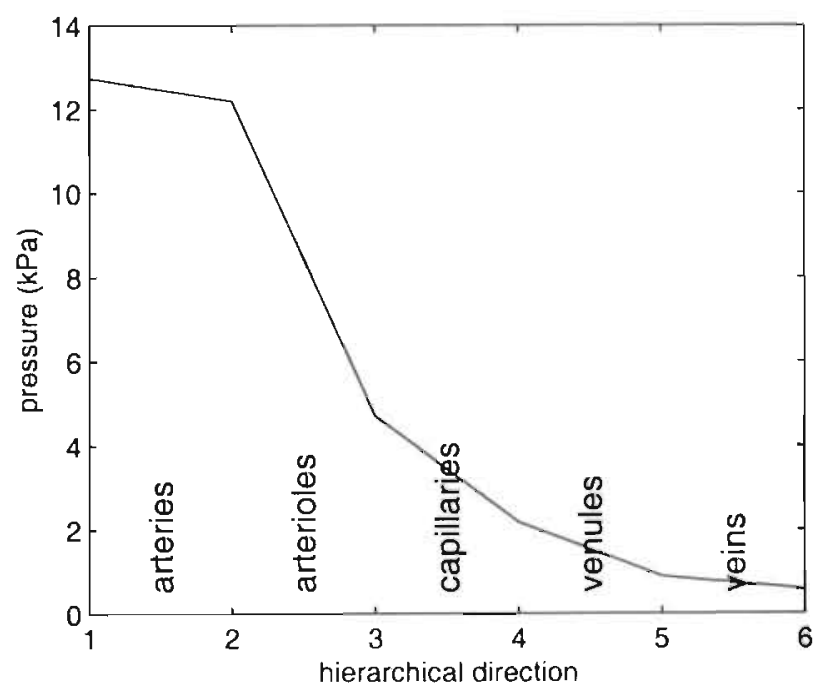


any point in space to the outflow orifice. If this assumption were wrong, and permeability at the macroscopic level of modeling is not homogeneous, the pathway for fluid flow becomes longer. Consequently, arterial and venous peak flows during contraction and total outflow volume may reduce significantly. A first approach to study this effect is to adjust the spatial distribution of heterogeneity and porosity.

The latter consideration is closely related to the aforementioned compartmentalization. If more compartments are included, then the heterogeneity within the large compartments increases. Yet, if the described effect of heterogeneity of the large vessels is actually important to muscle perfusion during contraction, then this can only be dealt with by increasing the number of compartments.

\section{Experimental procedure}

Between parts of experiments, $Q_{0}$ varied approximately $20 \%$. This is not expected to be the result of the condition of the rat, since flows did not steadily decrease with time, but varied randomly. Flow remained constant within one series of contractions. Most probably, variation was due to positioning of the probe around the vessel. The probe sensitivity is spatially non-homogenous, i.e. flow is under- or overestimated at certain locations within in the probe (1). However, the relationship between actual flow and measurement is linear. Therefore, comparison of flow ratios between experiments is allowed, whereas comparison of actual flow levels is not.

Peaks in flow signals can occur if a vessel is pulled through the probe. Two flow peaks would be registered if the vessel were pulled during contraction: one at the start of the contraction and one at the moment of muscle relaxation. For two reasons, the measured peaks are believed to be actual flow peaks, and not such movement-artifacts. First, it is expected that the muscle would pull the vessel during contraction and slides back during relaxation. However, the measured peak flow directions in Figure 2.1 and Figure 2.2 are the other way around. Second, arterial and venous flow peaks differ, whereas the described movement-artifact would produce nearly identical responses to the arterial and venous signals.

Muscle tension was altered using variations of both stimulation frequency and voltage within one experiment. Theoretically, voltage modulates the number of stimulated muscle fibers, whereas frequency determines the force that each stimulated fiber generates. Although differences may exist between voltage and frequency modulation with respect to intramuscular blood flow distribution, it did not affect the relationship between contraction and the gross flow signal. Therefore, these data are not separately examined, but treated as similar data. 
Finally, auto-regulation was allowed, no vasodilators were used, and perfusion pressure was not controlled, to leave normal muscle perfusion unaffected. Using $300-\mathrm{ms}$ contractions and a duty cycle of 0.3 , blood flow quickly raises to $50-70 \mathrm{ml} \cdot \mathrm{min}-1 \cdot 100 \mathrm{~g}-1$, which is 3 to 4 times $Q_{0}$. This means that $Q_{0}$ did not equal maximal flow level and that the vessels were not maximally dilated. Since within one contraction series $Q_{0}$ was comparable in the last few seconds prior to each contraction, it is assumed that vessels are at the same vaso-active state at that time. Arterial flow quickly reached a steady state during the contraction, and did not increase within $800 \mathrm{~ms}$. Therefore, vaso-regulation is thought to have only limited effects on blood flow within this time-period. Marshall and Tandon (63) showed that in response to tetanic contraction, only terminal arterioles were dilating within 3 seconds after the start of the contraction. Other arterioles and venules only started dilating a few seconds after a 1 -s contraction. Another reason to believe that the effect of auto-regulation is minimal is that after a contraction, a short period of increased arterial blood flow occurs. If this were the result of decreased vascular resistance due to vasodilatation, venous flow is expected to increase as well. The opposite is seen in the venous flow recordings. Furthermore, raised arterial flow and decreased venous flow during relaxation are also calculated by the FE model, which does not include auto-regulation.

\section{CONCLUSIONS}

Mean, systolic, and diastolic flow ratios decrease with active muscle tension. Mean flow during forceful contraction decreases to $10 \%$ of the mean flow level prior to the contraction (Figure 2.4). During diastole, arterial flow is arrested or inverted when muscle tension exceeds $80 \mathrm{kPa}$, but during systole, arterial inflow is present even during forceful contractions. The difference between systolic and diastolic flow ratio is constant, independent of active muscle tension (Figure 2.5).

Despite the strongly decreased perfusion, volume change during contraction is only $0.21 \%$ of muscle volume during contractions of $80 \mathrm{kPa}$ muscle tension (Figure 2.6). Volume change does not reach a steady state within an 800-ms contraction period.

Essential qualitative features like the in- and outflow peaks in the art(rial and venous signal during contraction and the characteristic venous flow pattern during muscle relaxation are present in both the experimental and the simulation flow signals. Quantitatively, flows differ. The most prominent differences regard blood volume change during contraction.

It is concluded from simulations that most initial volume change is located in the venous compartment, whereas the volume of other compartments 
steadily decreases when the contraction sustains. The finding that the most important effect of contraction on tissue perfusion is located in the veins is consistent with the vascular waterfall theory. 


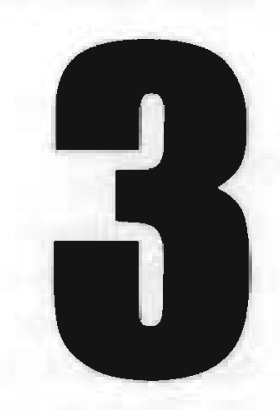

\section{SPATIAL INTERACTION BETWEEN TISSUE PRESSURE AND MUSCLE} PERFUSION DURING CONTRACTION

\footnotetext{
The finite element model of perfused skeletal muscle is used in this chapter to study the intramuscular behavior of the vascular waterfall during contraction. Spatial interactions between heterogeneous tissue pressure distribution, blood pressure and tissue perfusion are addressed.
} 


\section{INTRODUCTION}

Skeletal muscle blood flow increases 20-fold during dynamic muscle activity to adapt tissue perfusion to the required oxygen supply and waste matter drainage. Although during sustained tetanic contraction metabolic demands of skeletal muscle also increases, muscle perfusion is impeded (8, 43). The same holds for cardiac muscle perfusion during systole (60). The literature concerning the importance of intramuscular pressure in relation to perfusion during skeletal muscle contractions has recently been reviewed by Sejersted and Hargens (90). Several hypotheses relate delimited flow during tetanic contractions to intramuscular pressure rise. Local blood pressure is thought to rise with tissue pressure, as long as it exceeds local intravascular pressure. This phenomenon has been explained by the principles of flow through collapsible tubes (78). During periods of raised intramuscular pressure, an intravascular pressure drop from tissue pressure to systemic venous pressure occurs at the exact location where the draining vein leaves the muscle (28). Several studies $(22,61,85,94)$ have found evidence for the existence of this vascular waterfall showing that perfusion was independent of downstream venous pressure as long as venous pressure did not exceed tissue pressure.

In the aforementioned experimental studies, tissue pressure is controlled via a pressure box (95), which generates a homogeneous pressure. distribution. In contrast, intramuscular pressure distribution during muscle confractions is not homogeneous $(56,74)$, which may complicate the vascular waterfall behavior. Although tissue pressure and intramuscular blood flow distributions during muscle contractions: have never been measured simultaneously, subsequent. measurements suggest that blood flow distribution agrees with tissue pressure distribution $(56,121)$. The present study hypothesizes that due to heterogeneous tissue pressure distribution, local venous pressure not necessarily equals local tissue pressure as is assumed by the vascular waterfall theory.

To study vascular waterfall behavior during contractions, at least intramuscular pressure and venous pressure in intramuscular draining veins must be determined at a number of locations throughout the contracting muscle simuitaneously. Because of great difficulties of such experiments, the present: study uses: a numerical approach. Several numerical models have been developed to study the mechanical interaction between muscle contraction and perfusion. Most of these models have been used in cardiac mechanics $(5,23,28)$, and few for skeletal muscle research (21). An important shortcoming of these models is their lack of geometrical information, which is essential for realistic simulation of local mechanics. (18, 109). To study the mechanical interaction between blood flow and 
cardiac contraction, a porous media theory of blood flow in muscle tissue has been developed and implemented in a finite element description of contracting cardiac muscle by Huyghe et al $(51,52)$. Based on this concept, Vankan et al have developed a finite element model of blood perfused, contracting skeletal muscle tissue $(112,114,115)$. We recently demonstrated (116) that model predictions compare well to pressure box experiments performed by Shrier and Magder (94), thus clearly showing the presence of a vascular waterfall in simulations. The latter study also showed that the characteristic independence of perfusion from downstream venous pressure is less obvious during sustained contraction than under pressure box conditions.

The objective of the present study is to assess the aforementioned hypothesis that the heterogeneous distribution of intramuscular pressure during tetanic contraction interferes with the behavior of the generally accepted venous waterfall, making use of the finite element model of perfused contracting skeletal muscle.

\section{METHODS}

Two finite element simulations of blood perfusion in a two-dimensional representation of a unipennate muscle are performed. The geometry of the mesh is based on the midsagittal section of a rat medial gastrocnemius muscle (104). In the first simulation $\left(\mathrm{SIM}_{1}\right)$ one afferent artery and one efferent vein attach distally to the muscle model. In the second simulation $\left(\mathrm{SIM}_{2}\right)$, an additional pair of vessels is attached proximally (see Figure 3.1).

For finite element simulations, the software package DIANA (DIANA Analysis BV, Delft, The Netherlands) is used, in which a hierarchical mixture model for the description of blood perfusion in biological tissue has been implemented $(112,114,115)$. This mixture model consists of one solid constituent, representing skeletal muscle tissue, and a fluid

\section{Figure 3.1}

The two-dimensional finite element mesh is based on a longitudinal section through the medial gastrocnemius muscle. Thin lines indicate separate elements; thick lines are aponeurosis elements. The locations where arterial $(15 \mathrm{kPa})$ and venous $(0.6 \mathrm{kPa})$ pressures are prescribed (arrows) represent the locations where vessels

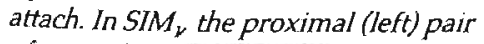
of prescribed pressures is absent.

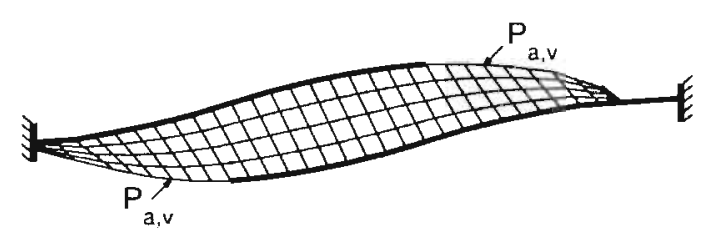


constituent that is subdivided into five inter-communicating fluid compartments, representing arterial, arteriolar, capillary, venular and venous blood. Regional and hierarchical pressure difference and local tissue permeability for spatial and hierarchical flow determine perfusion within and between subsequent blood compartments. Permeability depends on the non-linear relationship between local blood volume and the difference between local intra-vascular pressure and $\mathrm{P}_{\mathrm{IM}}$. This represents non-linear vessel wall stiffness and varies with hierarchical depth. Muscle tissue is described as non-linearly elastic, transverse isotropic passive material. Active stress in the muscle fiber direction can be prescribed to simulate a contraction. For an extended mathematical description of the model is referred to Vankan et al $(112,114,115)$.

The finite element mesh consists of 112 quadratic plane strain elements. Perfusion and contraction parameters were identical to those used by Vankan et al (116). 45 stiff elastic 1D truss elements represent the proximal and distal aponeuroses and the Achilles tendon. Figure 3.1 shows the mesh, including muscle fiber directions and the two nodes at which blood pressures (arterial: $15 \mathrm{kPa}$; venous: $0.6 \mathrm{kPa}$ ) are prescribed. The five most proximal nodes as well as the distal node of the Achilles tendon are fixed in all directions. An $800 \mathrm{~ms}$ contraction of $100 \mathrm{kPa}$ homogeneously activated muscle tension is simulated.

\section{RESULTS}

Figure 3.2 shows the capillary perfusion at rest $(A, B)$ and during contraction (C, D) as calculated for $\operatorname{SIM}_{1}(A, C)$ and $\operatorname{SIM}_{2}(B, D)$. At rest, capillary perfusion in both $\operatorname{SIM}_{1}$ and $\operatorname{SIM}_{2}$ is homogeneously distributed

Capillary flow

4
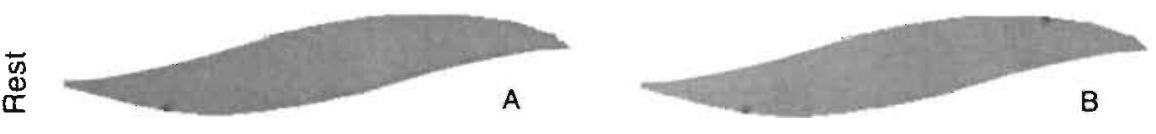

$\mathrm{mV} / \mathrm{min} / 100 \mathrm{~g}$

A

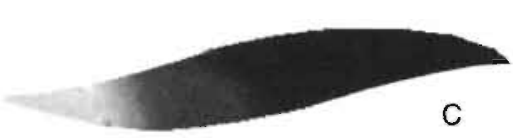

C
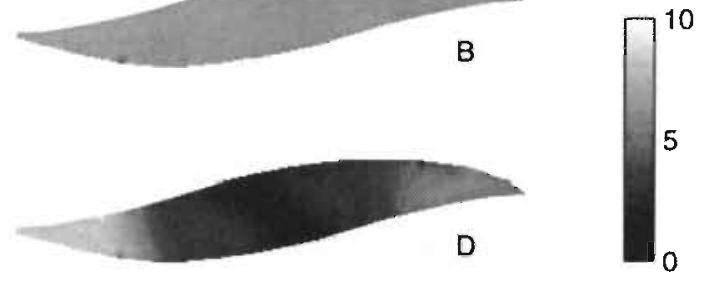

$\mathrm{SIM}_{1}$

$\operatorname{SIM}_{2}$

Figure 3.2

Capillary fiow through the muscle in the resting state $(A, B)$ and during contraction $(C, D)$ in SIM, $(A, C)$ and $S I M_{2}(B, D)$. At rest no difference in perfusion between the two situations exists. During contraction, flow decreased in the proximal muscle half without vessel ( $C$ ). 
To evaluate the distribution of the arterial-venous pressure difference, which is the driving force for capillary flow, arterial and venous pressures are visualized in Figure 3.4.

Arterial pressure is homogeneously distributed and rises slightly from $\sim 14 \mathrm{kPa}$ at rest to $\sim 16 \mathrm{kPa}$ during contraction in $\mathrm{SIM}_{2}$ and to $\sim 17 \mathrm{kPa}$ in $\mathrm{SIM}_{1}$. Venous pressure being similar at rest in $\operatorname{SIM}_{1}$ and $\operatorname{SIM}_{2}(\sim 2 \mathrm{kPa})$, strongly differs between the two simulations during contraction. In $\mathrm{SIM}_{2}$, venous pressure distribution merely follows the distribution of intramuscular pressure and reaches values of $\sim 7 \mathrm{kPa}$ during contraction in the center of the muscle. In $\operatorname{SIM}_{1}$, the distal muscle half resembles the pressure distribution in $\mathrm{SIM}_{2}$. In the proximal muscle half, venous blood pressure homogeneously rises to $\sim 17 \mathrm{kPa}$ in $\mathrm{SIM}_{1}$.

\section{DISCUSSION}

The results show that muscle perfusion at rest is independent of the number and position of afferent and efferent vessels. During contraction the attachment of these vessels becomes extremely important for the muscle capillary perfusion. With only one pair of vessels $\left(\mathrm{SIM}_{1}\right)$, capillary perfusion at the muscle side without feeding and draining orifice strongly decreases, unlike perfusion in $\mathrm{SIM}_{2}$ (Figure 3.2). The arterial-venous pressure difference, shown in Figure 3.4, explains this phenomenon. In $\mathrm{SIM}_{2}$, the arterial-venous pressure difference decreases from 14-1 to $17-7 \mathrm{kPa}$ during contraction, resulting in a global dccrease of capillary perfusion during contraction. In SIM , the proximal venous pressure rises to $17 \mathrm{kPa}$, reducing local arterial-venous pressure difference, and decreasing capillary perfusion (Figure 3.2).

Thus, the main cause of the decreasing perfusion is the increase in venous pressure, which, unlike expected from the vascular waterfall theory, exceeds local intramuscular pressure in a large part of the muscle. Due to vessel compression in the muscle center, central local venous pressure follows the local intramuscular pressure rise, which is in agreement with the vascular waterfall theory. The high venous pressure in the central region prevents proximal venous blood from flowing towards the draining vein in the distal muscle half. Proximal venous blood only passes the central band of high venous pressure if proximal blood pressure exceeds the central pressure. Meanwhile, blood flow in the arterial-venous direction continues in the proximal muscle, and local venous pressure increases. Further filling of veins stops when local venous pressure equals arterial pressure, or the local venous pressure exceeds the central venous pressure. The result is shown in Figure 3.3, where proximal venous blood pressure rises to arterial pressure levels. 
These two simulations illustrate that venous pressure follows the rise in intramuscular pressure, according to the vascular waterfall theory. Due to spatial interactions between intramuscular pressure and blood pressures, venous pressure importantly rises independently from the local intramuscular pressure in a large part of the muscle. This results in local tissue underperfusion during contraction, which may affect muscle functioning.

It is concluded that regional effects are important to understand muscle functioning. The prediction of the vascular waterfall theory that venous pressure is determined by intramuscular pressure should not be followed without considering geometrical effects, as spatial interactions can principally influence skeletal muscle blood pressure distribution.

Furthermore, it can be concluded that the number and positions of veins rather than arteries are responsible for the shown perfusion problems during contraction. Flow is impeded not because of a lack of perfusion from the arterial side, but because the venous blood is trapped as it cannot reach the distal draining vein. Adding an extra proximal draining vein, like in $\mathrm{SIM}_{2}$ is sufficient for proper muscle perfusion. An additional artery does not sort the same effect.

A specific fiber orientation or geometry could probably prevent the intramuscular pressure from forming a high-pressure band by which the blood is trapped. Large differences in intramuscular pressure rise measured in different muscles supports the idea that muscle geometry and fiber organization are responsible for large variations in pressure building during contraction (90). For instance, intramuscular veins located in lowpressure areas in the muscle could provide a pathway for the trapped venous blood to bypass the central high-pressure area and reach the outflow orifice.

It is recommended to further explore the present conclusion that spatial interactions must be considered for better understanding of muscle perfusion during tetanic contraction, in an experimental setting. 

C.C. van Donkelaar, P.J.B. Willems,

A.M.M. Muijtiens and M.R. Drost.

Skeletal muscle transverse strain

during isometric contraction at

different lengths, Journal of

Biomechanics, 1999. accepted.

(Copyright 1999, Elsevier Science LTD).

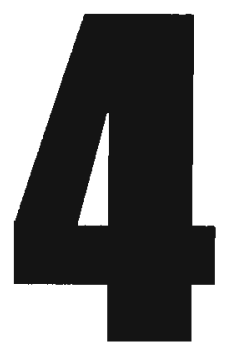

\section{SKELETAL MUSCLE TRANSVERSE STRAIN DURING ISOMETRIC CONTRACTION AT DIFFERENT}

LENGTHS

The plane strain assumption, often used in twodimensional numerical models of skeletal muscle contraction, is studied with respect to muscle deformation. Therefore, transverse strain at the muscle surfaced is determined from three-dimensional marker displacements during isometric contractions at various muscle lengths. 


\section{INTRODUCTION}

The basic function of skeletal muscles is to generate movement. Therefore, they can produce force at different lengths and velocities. Force-length and force-velocity curves are well-known descriptions of mechanical muscle functioning that incorporate only one spatial dimension. To study the effects of contraction on muscle fiber length, aponeurosis length or pennation angle, this approach is insufficient. Therefore, a two-dimensional representation of for instance the midsagittal muscle plane is often used to study muscle deformation $(33,75,109,116,117,122)$. Except for the model of Gielen (33), who used a nearly plane stress approximation, these models use plane strain descriptions to calculate deformation. The assumption of constant muscle volume during contraction is effectuated in these plane strain models by assuming constant muscle longitudinal sectional area. Thus, longitudinal shortening is only accounted for by increasing muscle size in the second model dimension, whereas deformation in the third dimension is suppressed. In contrast, free deform in the third dimension is allowed in plane stress simulations.

Gielen et al (33) compared plane stress and plane strain approximations in finite element simulations of muscle contraction. Their results show distinct differences in tissue pressure and strain in all directions, which stresses the importance of these model assumptions regarding deformation of the twodimensional muscle plane.

This plane strain assumption is experimentally challenged by Zuurbier and Huijing (125), who found that the area of the midsagittal plane of the medial gastrocnemius muscle decreases $24 \%$ during isometric contraction. However, their experimental procedure is questionable, because it is based on two-dimensional measurements of marker locations, not accounting for muscle rotation, and the midsagittal plane area is determined by interconnecting four markers at the approximate proximal and distal endings of the aponeuroses with straight lines, and not from the actual muscle outline.

Another way to study the plane strain assumption is by actually measuring strain perpendicular to the midsagittal muscle plane during isometric contraction. Only few studies measured this transverse muscle surface strain in aponeurosis and superficial muscle fibers. During passive muscle stretching, transverse strains in both tissue types are in the order of $6 \%$ (102). During contraction, Scott and Loeb mentioned increasing aponeurosis width, without further quantification (89), whereas Van Bavel et al reported very variable transverse strains, decreasing from the central band towards the edges (103). They discussed that the variability probably results from projection errors in their two-dimensional measurements. 
Strong curvature in the transverse muscle direction requires threedimensional measurements to avoid projection errors in transverse muscle surface deformation. Such artifacts involved results of previous deformation studies $(46,89,101,102,119,124)$, although they are of minor importance in the longitudinal muscle direction.

Deformation of aponeurosis and muscle fibers in the longitudinal muscle direction was extensively studied. Longitudinal muscle fiber strains are in the order of $15 \%(37,103)$. Aponeurosis lengthening during passive stretching (59, 101, 102) and isometric contraction $(46,89,103,119)$ was generally in the order of 1-5\%. Zuurbier et al agreed to these values in the central part, but reported much larger strain in the proximal $(10 \%)$ and distal $(52 \%)$ part of the proximal gastrocnemius aponeurosis (124).

Although part of the differences, reported in the literature The small longitudinal aponeurosis strain is explained by the stiff, non-linear material properties in that direction (89). Because in the longitudinal direction, aponeurosis tissue is stiffer than passive muscle fibers, passive stretching of a skeletal muscle affects muscle fiber length more than aponeurosis length. Consequently, muscle surface strain distribution and especially the relationship between aponeurosis and superficial muscle fiber strain, will depend on initial muscle length.

The objectives of the present study are to accurately quantify transverse muscle surface deformation during contraction, based on threedimensional measurements, in order to study the model assumption of plane strain deformation. Therefore, the relation between transverse and longitudinal skeletal muscle surface strain in superficial muscle fibers and the aponeurosis are compared. For the expected effect of muscle length on muscle deformation, longitudinal and transverse strain is determined during contractions at different initial lengths.

\section{METHODS}

\section{Experimental setup}

Three male, 12 weeks old Lewis rats were anaesthetized with sodiumpentobarbital (Numbutal ${ }^{\oplus}, 0.1 \mathrm{ml} / \mathrm{kgBW}$, i.p.) after short-term $(<20 \mathrm{~s})$ sedation with $\mathrm{CO}_{2}$. Sodium-pentobarbital was supplemented as necessary. The local ethical committee approved the experiments. During the preparation and the experiment, the rat was placed on a heated metal table $\left(37.0 \pm 0.1^{\circ} \mathrm{C}\right)$. After removing the skin of the left hind leg below the knee, the muscle surface was frequently sprayed with physiological saline solution. The fascia was carefully removed from the medial surface of the medial gastrocnemius muscle. Approximately 70 fluorescent polystyrene 
spheres (Bangs Laboratories Inc. Fishers, USA) of $0.45 \pm 0.05 \mathrm{~mm}$ diameter were attached to the tissue with histoacryl tissue glue (Histoacryl blau, Braun, Melsungen). Van Bavel et al showed that approximately $70 \%$ of the marker diameter was glued to the muscle surface with this procedure (102). Extrapolated to the present study, less than $0.1 \%$ of the medial muscle surface is glued, which is expected to hardly affect total surface deformation. Spheres were uniformly distributed over the medial surface with an interdistance of $\sim 3 \mathrm{~mm}$. By gently pulling each marker and observing underlying tissue deformation under a stereo microscope, it was verified that the marker was glued on muscle tissue and not on sparse remains of the fascia, which would severely affect deformation (89).

After dissecting the triceps surae muscle and the calcaneus bone free from the tibia and the foot, the calcaneus bone was fixed to the force transducer in the upper traverse of a draw-bench (Zwick 1445, Zwick GmbH, Ulm, Germany). Accuracy of the force transducer was $0.02 \mathrm{~N}$. Two pairs of tweezers, slid through the tractus iliotibialis, firmly attached the femur horizontally to the lower traverse. The muscle was positioned in the drawbench such that the line of pull was exactly vertical. Care was taken not to rotate the muscle along its longitudinal axis. In this way, the attachment of the muscle to the femur was comparable to the in vivo situation in which the knee was bent 90 degrees. By elevating the upper traverse of the drawbench, muscle length could be adjusted with an accuracy of $0.01 \mathrm{~mm}$.

Two electrode wires (Biomed wire, Cooner wire, Chatsworth, Ca) were gently wrapped around the sciatic nerve for electrical stimulation of the muscle with a pulse generator (HSE 215/IZ, Freiburg, Germany). Two

Figure 4.1

Right camera image after the experiment with normal backlight and without the filter in front of the lightsource to show both the markers and the muscle surface. The calcaneus clamp (black horizontal bar) and the Achilles tendon are visible at the top of the figure. The faint white proximal area (bottom) is part of the aponeurosis.

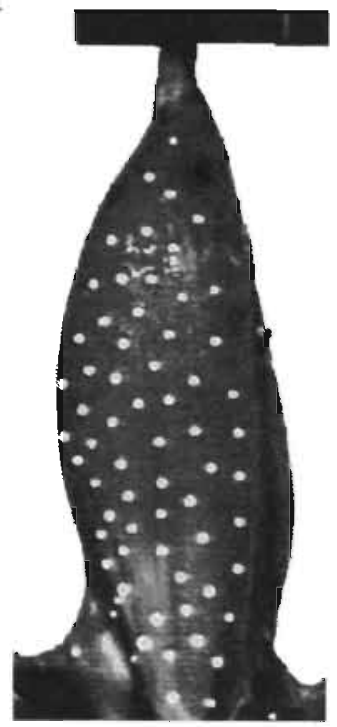


synchronized $25 \mathrm{~Hz}$ CCD cameras (MX5, Adimec, The Netherlands) with a 75-mm lens (Ernitec F1.3 TV) were placed in front of the muscle. The angle between the optical axes of the cameras, which intersected at a central point in the workspace, was $60.6^{\circ}$. The line connecting the two lens centers was defined as the $\mathrm{x}$-axis. The $\mathrm{y}$-axis was perpendicular to the $\mathrm{x}$-axis through the intersection point of the optical axes of the cameras. The $z$-axis was perpendicular to the $x$ - and $y$-axis, and thus parallel to the longitudinal muscle axis. To optimize detection of the fluorescent markers, a blue filter $(450 \mathrm{~nm}$ ) was placed in front of the light source (KL 1500 electronic, Schott, Wiesbaden, Germany) and a yellow filter $(600 \mathrm{~nm})$ was placed in front of the camera. Shutter time was $3.2 \mathrm{~ms}$, F-stop was 4 and Lightning was manually adjusted for best marker contrast.

Force was processed (sample frequency $1000 \mathrm{~Hz}$ ) via an 8-channel, 12-bits Lab-NB AD conversion board (National Instruments), programmed with LabVIEW. Force signals were used to determine optimum length and to keep track of the force magnitude during the experiment. Video images $(25 \mathrm{~Hz}$ ) were real-time digitized and stored via the $R$ and $G$ input of an 8-bit RGB frame-grabber (DMA Magic, Matrox, UK, 400*512 pixels), programmed with DMA Magic (DIFA measuring syst'ms, Breda, The Netherlands). An example of a camera view of the muscle with attached markers is shown in Figure 4.1.

\section{Experimental procedure}

The force transducer was zeroed at very short muscle length. Muscle length was then increased to the length at which $0.5 \mathrm{~N}$ force was measured. From pilot experiments it was known that this length was just below optimum length $\left(\mathrm{L}_{0}\right)$, defined as the length at which the muscle can generate maximum active force. At this length, stimulation voltage and frequency were optimized for maximum active force using $100 \mathrm{~ms}$ contractions. Optimized for maximum force, stimulation voltage was in between 0.7 and

Figure 4.2

A sample tracing of the force signal, recorded during the experiment. Sample frequency is $1000 \mathrm{~Hz}$.

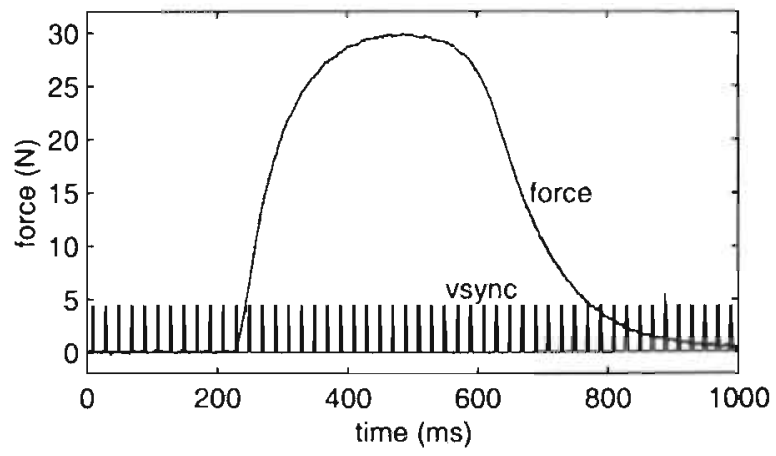


$0.9 \mathrm{~V}$ and frequency in between 80 and $90 \mathrm{~Hz}$. An example tracing of the force signal is shown in Figure 4.2.

Isometric $350 \mathrm{~ms}$ contractions were performed. During each contraction, $600 \mathrm{~ms}$, video and force recordings were obtained. Acquisition started $80 \mathrm{~ms}$ prior to contraction. In between contractions, the muscle was lengthened or shortened by $0.5,1,1.5$ or $2 \mathrm{~mm}$ at a time, in arbitrary order. At least 2 minutes were interposed between subsequent contractions. $\mathrm{L}_{0}$ was determined during the experiment. All contractions were performed at lengths between $4 \mathrm{~mm}$ shorter and $3 \mathrm{~mm}$ longer than $\mathrm{L}_{0}$.

\section{Data processing}

Geometrical marker centroids were determined from the digitized image frames using TIMwin 1.3 (TEA Dordrecht, 1993). Three-dimensional marker tracks, i.e. three-dimensional positions for each marker in time, were reconstructed from these centroids. Therefore, marker tracks per camera were determined from the marker coordinates in the individual images (71). Markers that were occasionally missing in a single image were automatically filled in by marker track interpolation (69). In practice, this concerns maximally 2 out of 30 images per marker track, and maximally 3 out of $\sim 70$ marker tracks per contraction. Stereoscopic pairs of tracks in the two views were identified on the basis of minimum stereoscopic mismatch (70). Finally, the geometry of the stereoscopic camera set-up was determined from these pairs, and the corresponding three-dimensional marker tracks were reconstructed (68). From the actually applied 70 markers, typically 55 to 65 tracks could be reconstructed with this automated procedure. Most missing tracks were lateral markers that were not continuously visible in both the cameras.

Strains were calculated from marker displacements between the first image of the data acquisition, recorded $80 \mathrm{~ms}$ prior to the contraction, and the first image during the steady state of the contraction. The marker centroids in these images were calculated from a subsequent odd and even image frame together, using a pixel-intensity weighted procedure to increase accuracy to $1 / 20^{\text {th }}$ pixel, which corresponds to $-5 \mu \mathrm{m}$.

Local Green-LaGrange strains at each marker position were calculated in the plane, fitted through the coordinates of the strain group markers, according to the procedure described by Peters (79). This method is based on the assumption of linear strain fields within a strain group, defined as a circle $(\mathrm{r}=3.5 \mathrm{~mm})$ around the marker. Note that due to these strain groups, strain distribution is subject to a low-pass filtering effect. Decreasing strain group size increases the ability to detect spatial heterogeneity, but strain calculated from a small number of markers becomes inaccurate (79). Strain groups containing less than 5 markers, excluding the central marker, were 
excluded from analysis to calculate reliable strain in a sufficient number of strain groups.

Markers were separated into one group that had been attached to the aponeurosis and one that had been attached to the muscle fiber surface using a stereo microscope. Borderline markers at the sometimes-unclear transition between aponeurosis and muscle fibers were not included in these marker sets. For quantitative analyses, strain calculations were performed on the separate sets to account for the otherwise blurred transition area between aponeurosis and muscle fibers. Although this increases the number of edge-markers, which are less accurately calculated, the introduced inaccuracy is less erroneous than low-pass filtering the sharp transition in longitudinal strain in the transition area between aponeurosis and muscle tissue.

Accuracy of data processing, from the rough image acquisition to the strain calculations is within $0.2 \%$. This has been estimated from strain computation at marker positions between two subsequent odd fields of a resting muscle. Longitudinal strains were $0.02 \pm 0.11 \%$, transverse strains $0.06 \pm 0.19 \%$.

Longitudinal strains were defined as strains parallel to the local projection of the muscle axis on the muscle surface and were calculated by rotating the Green-LaGrange strain matrix. Transverse strain was perpendicular to the longitudinal strain, parallel to the muscle surface. Surface area-change

Figure 4.3

A: projection of all three-dimensional marker tracks during a contraction. $B$ : lines represent projections of principal strains at the muscle surface, calculated from the marker tracks shown in Figure 4.3A. Fat lines represent positive strains, thin lines negative strains. Angles between principal strain vectors deviate from 90 degrees if they are not parallel to the plane of projection (xz-plane). The plotted vector length represents the magnitude of the principal strain. The vector length corresponding to $10 \%$ strain is indicated in the figure. The circle represents the strain group size.
Marker tracks

Principal strain

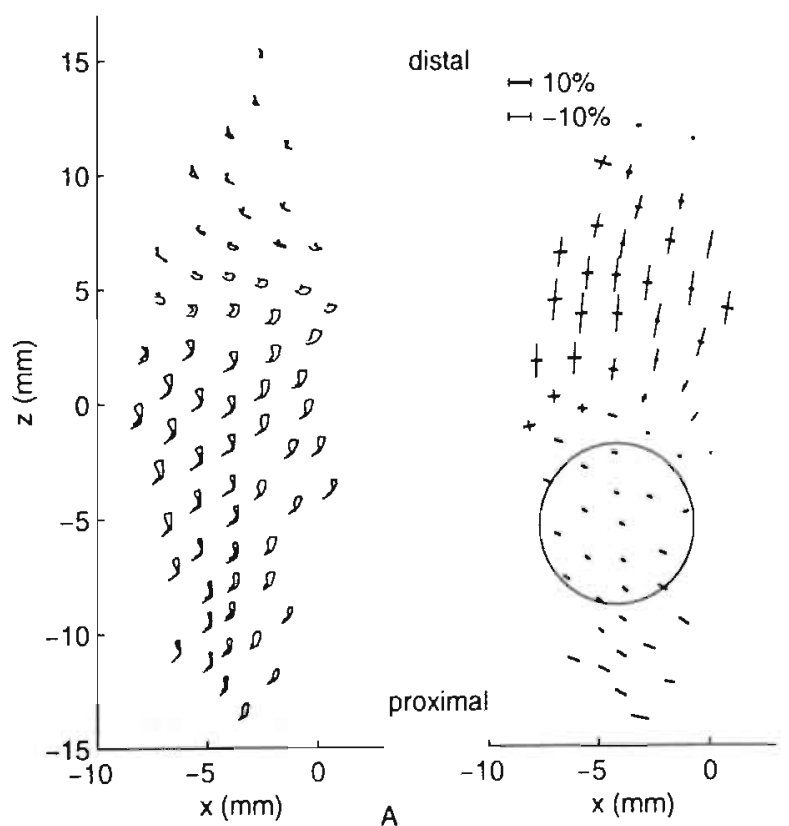


was determined from multiplication of the elongations, calculated from the principal strains, per marker.

\section{RESULTS}

An example of three-dimensional marker tracks, projected in the xz-plane (Figure $4.3 \mathrm{~A}$ ) and the projection of the three-dimensional principal strain vectors at the muscle surface, derived from these tracks (Figure $4.3 \mathrm{~B}$ ), show a clear difference between muscle fibers (negative principal strains in the approximate fiber direction in between -5 and $-20 \%$ ) and the aponeurosis (strains in the order of $+1 \%$ ). The difference between the tissue types is also illustrated from the longitudinal strain distribution (Figure $4.4 \mathrm{~A}$ ), but is not seen in the distribution of transverse strain, varying between 0 and $10 \%$ in both muscle fibers and aponeurosis (Figure 4.4 B). These strains were calculated from the displacements of all markers, without separating aponeurosis and muscle fiber markers. The smooth transition between aponeurosis and muscle fiber strain near the center of the image is the

\section{Figure 4.4}

A: distribution of longitudinal strains at the muscle surface. Longitudinal strain clearly differs between muscle fibers (top) and aponeurosis (bottom), although the figure shows a vague transition between those tissue types, due to the use of strain groups. $B$ : distribution of transverse strains at the muscle surface. The distributions of aponeurosis and muscle fibers transverse strains overlap. These color plots are computed from cubic interpolations of the marker strains in a $0.3^{*} 0.3 \mathrm{~mm}$ grid. The plotted area represents the smallest outline surrounding all markers, and not the actual muscle contours. All values are given in percentages.
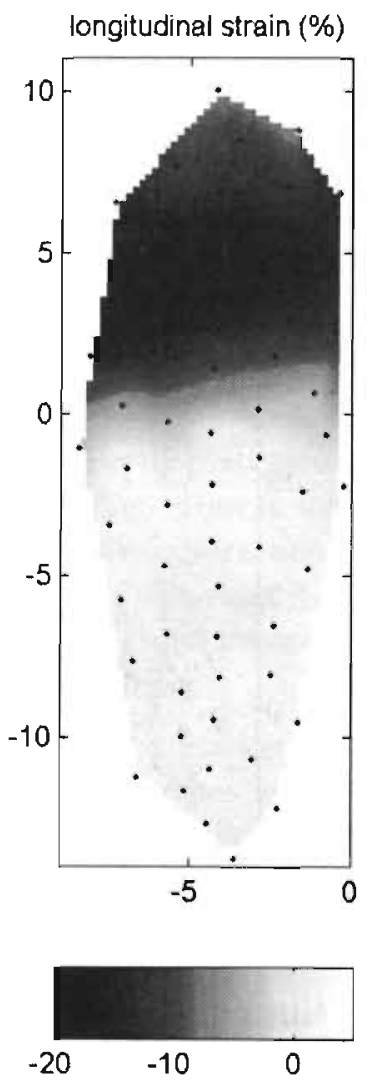

transverse strain (\%)

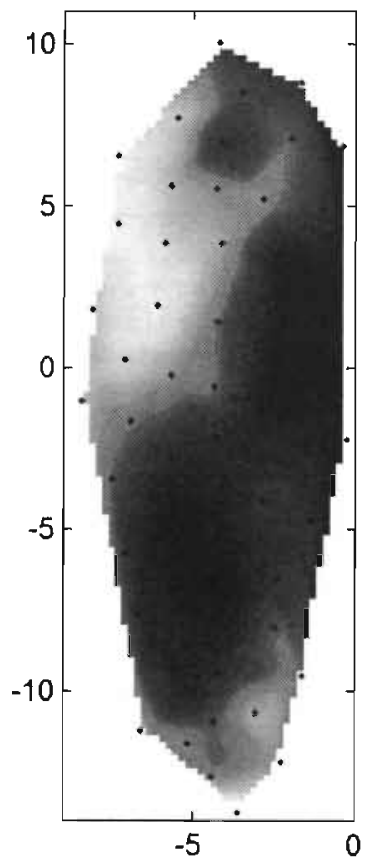

A

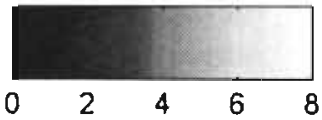


result of the previously discussed low-pass filtering effect, due to the use of strain groups.

Mean transverse and longitudinal strains of markers in the superficial muscle fibers and the aponeurosis are calculated for each contraction, based on separate sets of aponeurosis and muscle fiber markers. These strains as well as the average local surface area-change during contraction are plotted in relation to initial muscle length, which is shown relative to optimum muscle length (Figure 4.5). Although intercepts of the regression lines through these data differ between experiments, slopes are comparable (Table 4.1).

\section{Figure 4.5}

Mean longitudinal $(A, B)$ and transverse $(C, D)$ strains and areachange ( $E, F)$ of the muscle tissue ( $A$, $C, E)$ and the aponeurosis $(B, D, F)$ are plotted against muscle length during contraction. Each symbol equals the mean strain or area change at a certain muscle length. Bars indicate the standard deviations of these values. These are data from all three animals, each one with a different symbol. Lingth in $\mathrm{mm} ; 0 \mathrm{~mm}$ equals optimum muscle length.
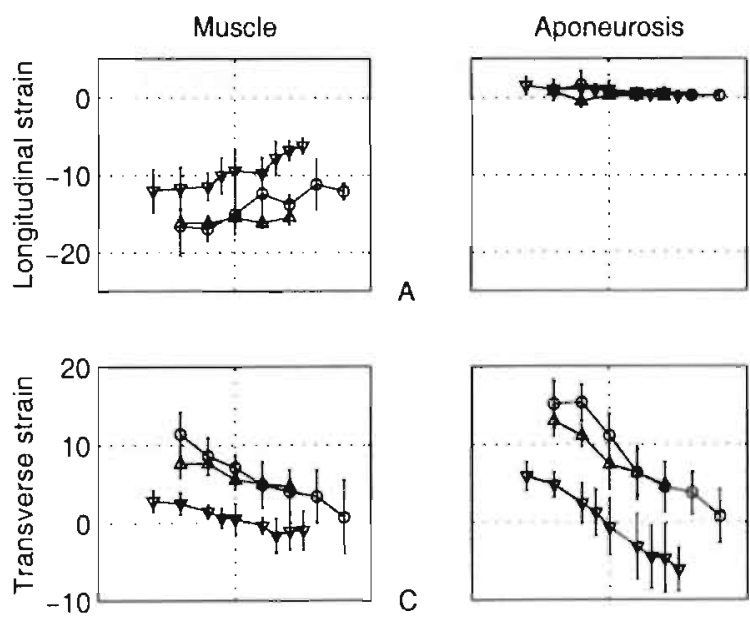

D
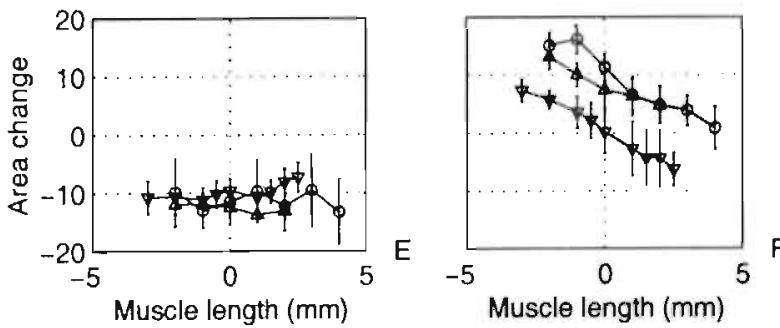

$F$

\section{Table 4.1}

Means and standard deviations

$(\because \mathrm{mm}, \mathrm{n=3})$ of the slopes that belong to the relationships between longitudinal strain, transverse strain and surface area-change in muscle fibers and aponeurosis on the vertical axis, and muscle length on the horizontal axis, as shown in Figure 7.5.

\begin{tabular}{|l|l|l|l|}
\hline $\begin{array}{l}\text { Slopes } \\
(\% / \mathrm{mm})\end{array}$ & $\begin{array}{l}\text { Longitudinal } \\
\text { strain }\end{array}$ & $\begin{array}{l}\text { Transverse } \\
\text { strain }\end{array}$ & $\begin{array}{l}\text { Surface } \\
\text { area-change }\end{array}$ \\
\hline Muscle fibers & $0.72 \pm 0.41$ & $-1.08 \pm 0.37$ & $-0.02 \pm 0.40$ \\
\hline Aponeurosis & $-0.15 \pm 0.06$ & $-2.35 \pm 0.20$ & $-2.41 \pm 0.27$ \\
\hline
\end{tabular}


The independence of muscle surface area-change in relation to initial muscle length (Figure 4.5) is also visible from the relationship between mean transverse and longitudinal muscle fiber strain of all contractions in three muscles $\left(\mathrm{E}_{\mathrm{\gamma}}=-0.98^{\star} \mathrm{E}_{\mathrm{L}}-8.67 \%, \mathrm{r}^{2}=0.83, \mathrm{n}=21\right)$, shown in Figure 4.7. The slope of -0.98 indicates that all change of muscle length is accounted for by muscle fiber transverse strain.

Finally, aponeurosis and muscle tissue transverse strain of all contractions in three muscles highly correlate $\left(\mathrm{E}_{\mathrm{T} \text {,mus }}=0.54^{\star} \mathrm{E}_{\mathrm{T} \text {,apo }}+1.06(\% / \mathrm{mm}), \mathrm{r}^{2}=0.95\right.$, $\mathrm{n}=21$, see Figure 4.6). The slope indicates that the aponeurosis is stretched or compressed twice as much as the muscle tissue in the transverse direction.

Figure 4.7

Transverse $\left(E_{\gamma}\right)$ vs. longitudinal $\left(E_{\nu}\right)$ muscle fiber strain. Data points are mean strain values of contractions at different muscle length of three animals. The regression equation equals $E_{T}=-0.98^{*} E_{L}-8.67(\%), r^{2}=0.83$.

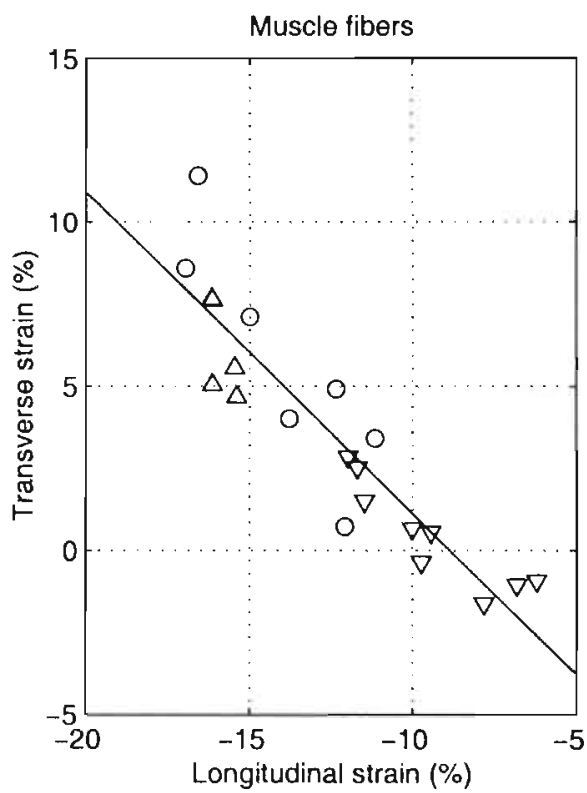

Figure 4.6

Mean transverse strains of the muscle tissue ( $E_{\text {Tmus }}$ - vertical axis) are plotted against transverse strains of the aponeurosis ( $E_{T, \text { apo }}$ - horizontal axis). Data points result from contractions at different lengths, each symbol represents an animal. The regression equation equals

$E_{T \text { mus }}=0.54^{*} E_{T \text {,apo }}+1.06(\% / \mathrm{mm})$, $r^{2}=0.95$.

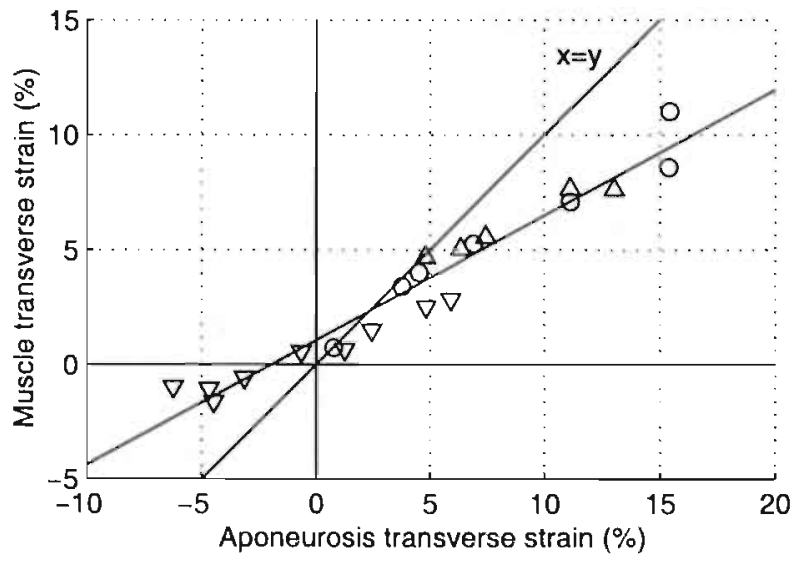




\section{Interpretation of results}

Surface strains at the medial side of the medial gastrocnemius muscle are computed from three-dimensional measurements of marker displacements. As expected, muscle tissue and aponeurosis show distinctive differences in longitudinal strain (see Figure 4.4). Longitudinal strains for muscle fibers ($14 \pm 2.6 \%$ at $L_{3}, n=57$ ) are in agreement with those reported in the literature (37, 103). Aponeurosis longitudinal strain of $0.8 \pm 0.9 \%$ at $L_{0}$ is at the lower end of the generally reported $1-5 \%(46,89,103,119)$. A predefined strain distribution was not found. Regarding the aponeurosis, this agrees with Scott and Loeb (89), but others found decreasing aponeurosis strain in the proximal-distal $(46,103)$ or in the opposite direction (124).

Transverse strain in superficial muscle fibers $(4.8 \pm 2.8 \%, \mathrm{n}=57)$ and aponeurosis $(6.1 \pm 4.5 \%, \mathrm{n}=72)$ at $\mathrm{L}_{0}$ (Figure 4.4 ), are comparable and in agreement with the reported $6 \%$ for passive stretching by Van Bavel et al (103). Yet, their conclusion that transverse strain in superficial muscle fibers and in aponeurosis is similar, is not generally true for all isometric contractions (Figure 4.6). For instance, only with aponeurosis transverse strain between -2.0 and $6.7 \%$, the difference between mean muscle and aponeurosis transverse strain is smaller than 2\% (Figure 4.6). Because transverse strain changes with muscle length (Figure $4.5 \mathrm{C}, \mathrm{D}$ ), it can be concluded that at both long and short muscle lengths, transverse strains during isometric contraction differ between superficial muscle fibers and aponeurosis, whereas at intermediate muscle length, they are comparable.

The results further show that independent of muscle length, the muscle surface area changes with $-11 \%$ (Figure 4.5E). Assuming incompressible muscle tissue, strain perpendicular to the muscle surface therefore equals $11 \%$ during isometric contractions at all muscle lengths.

Translated to a representative superficial muscle fiber, these results indicate that strains in the two directions perpendicular to its longitudinal direction, are not alike during contraction. Strain perpendicular to the muscle surface equals $11 \%$ independent of muscle length, whereas strain parallel to the muscle surface varies $\sim 15 \%$, depending on muscle length (Figure $4.5 \mathrm{C}$ ). From these findings follows that transverse surface strain, and not strain perpendicular to the surface, compensates for changes in longitudinal strain with different muscle length, which can also be derived from slope of the relationship between transverse and longitudinal muscle strain $(-0.98$, Figure 4.7). Because transverse muscle surface strain changes with muscle length, it can be concluded that plane strain models, which do not account 
for deformation in the transverse direction, cannot be used to calculate the effect of muscle length changes on deformation during contractions.

In Figure 4.6 it is shown that at a certain muscle length, transverse strain is approximately $0 \%$ in both aponeurosis and muscle fibers. This corresponds to contractions at the muscle length in which longitudinal strain is approximately $-9 \%$ (Figure 4.7). These results do not counteract the use of plane strain descriptions in simulations of isometric contraction at this particular muscle length.

With $11 \%$ strain perpendicular to the muscle surface, independent of muscle length, and transverse strain parallel to the surface changing with longitudinal strain, the fiber cross-section aspect ratio changes, depending on longitudinal fiber strain. If transverse strain and strain, perpendicular to the muscle surface were equal, muscle fibers would restrain their aspect ratio. From the relationship between transverse strain and longitudinal strain (Figure 4.7), it is derived that transverse strain equals $11 \%$ only when longitudinal strain reaches approximately $-20 \%$. Even in short muscles, transverse strain does not decrease below $-18 \%$. It is therefore concluded that superficial muscle fibers flatten during isometric contraction.

\section{Experimental procedure}

In the present study, the triceps surae muscle was isolated from surrounding tissues and fixed in a draw-bench to enable simultaneous measurement of muscle force and deformation, whereas muscle length can accurately be adjusted. Other studies used comparable experimental setups to determine muscle force $(13,31,89,102,119)$. Two remarks regarding this procedure and extrapolation of results to in vivo contractions follow. First, removal of surrounding muscle and bone may involve muscle deformation. As measurements were performed at the dorso-medial surface, away from the supporting tibia bone and other muscles, the effect on local deformation is considered minimal. Second, the fascia is removed to prevent attached markers from sliding over the muscle tissue. Although the fascia around the medial gastrocnemius muscle is less developed compared to the fascia of e.g. the tibialis anterior muscle, it might still restrict muscle swelling and therewith limit transverse strain in vivo. This issue should be studied in future research using for instance tagging-MRI methods.

Finally, the longitudinal muscle direction fairly accurately represents local muscle fiber direction in the central band of the muscle, from the most distal part of the proximal aponeurosis to the Achilles tendon. Near the lateral attachment of fibers to the aponeurosis, local fiber directions deviate up to $15^{\circ}$ from the longitudinal direction. This does not affect the present results, but it is important to consider when interpreting the results as if they represent strain in individual muscle fibers. 
C.C. van Donkelaar, M.R. Drost, $H$. van Mameren, C.F. Tuinenburg J.D. Janssen, A. Huson, Threedimensional reconstruction of the rat triceps surae muscle and finite element mesh generation of the medial gastrocnemius muscle, European Journal of Morphology, 34(1): 31-37, 1996.

(Copyright 1996, Swets \& Zeitlinger Publishers).

\section{THREE-DIMENSIONAL RECONSTRUCTION AND FINITE ELEMENT MESH GENERATION OF RAT CALF MUSCLE}




\section{INTRODUCTION}

Blood flow through muscle is important for oxygen supply and transport of metabolites. Isometric endurance time of tetanic skeletal muscle contraction increases by increasing perfusion pressure, showing the importance of mechanical variables for perfusion (80). Several models concerning the mechanical behavior of muscle during contraction have been published ( 47 , $107,109,122)$, but these do not take changes in blood flow as a result of intramuscular pressure and muscle deformation into account. Our group developed a finite element model of blood perfused skeletal muscle that calculates deformation, intramuscular pressure, blood pressures and blood flow in different blood compartments in contracting perfused skeletal muscle (113). This model is implemented in the finite element software packige DIANA (DIANA analysis BV, Delft, The Netherlands). Functioning of the medial gastrocnemius muscle (GCM) is studied with this model, because much research that can be used to validate the model has already been carried out regarding this muscle. Regional blood flow distribution during contraction was measured by Pendergast et al (77) and Piiper et al (82) while measurements of deformation during contraction were performed by Zuurbier et al (126) and Van Bavel et al (102).

Accurate calculation of deformation and perfusion during contraction, calculated with the finite element method, requires incorporating mechanically important aspects of the GCM geometry. This includes the geometry of the muscle belly and the aponeuroses as well as muscle fiber directions. Several studies determined aspects of the GCM geometry (44, $107,109,122)$. However, their geometrical descriptions are not detailed enough for accurate finite element simulations. The present study aims at creating a finite element mesh of the GCM. A finite element mesh is build with a number of interconnected nodes that form elements. The more details are to be included into a mesh, the more elements and nodes have to be used. Calculation time increases with the number of elements. Simulation time of a forceful tetanic muscle contraction on a SUN Spark Classic, using a mesh built with approximately 1000 elements, is acceptable.

Since the GCM cannot be dissected from the lateral gastrocnemius muscle (GCL) without damaging the muscle distally, a precise histological reconstruction of the rat triceps surae muscle, visualized by means of computer graphical techniques, is accomplished in advance. Outlines of the GCM and the geometry and thickness of accompanying aponeuroses are identified in this reconstruction and used to actually generate the finite element mesh. As an example of the use of the generated finite element mesh, passive perfusion is calculated in a companion paper (113). 
The aim of the present study is to generate a detailed geometrical reconstruction of the GCM, which is to be used in a finite element analyses. This is preceded by a detailed histological reconstruction of the triceps surae muscle, using computer graphical techniques.

\section{METHODS}

\section{Triceps surae muscle reconstruction}

To obtain a triceps surae muscle for a reconstruction, the distal part of the tibia and the foot of an anaesthetized 12 weeks old male Lewis rat were fixed. The angles of both the knee and the ankle were set at 90 degrees. The rat was perfusion fixated with a buffered formaldehyde solution ( $\mathrm{pH}$ 7.2), injected into the left ventricle. X-ray images of the hind limb were obtained after the perfusion fixation to verify the angle of the knee and the ankle joint. The triceps surae muscle, including GCM, GCL, plantaris (PL) and soleus muscle (SOL), was dissected free from its surroundings. The distal tendon was cut near the calcaneus and the muscles were carefully removed from their origins. The muscle was dehydrated and embedded in a solution of Technovit 7100 with $0.2 \%$ polyethyleneglycol. It was longitudinally surrounded by 5 parallel graphite bars $(0.3 \mathrm{~mm}, 2 \mathrm{~B})$ that were attached in small plastic containers, positioned at both sides of the muscle. Out of 8000 cross-sections $(4 \mu \mathrm{m})$ through the complete length of the muscle, obtained with a microtome (2218 Historange), 80 slides were colored with trichrome Gomorri for 3 hours, to discriminate between collagen of aponeuroses and muscle fiber tissue. As a result of the mentioned graphite bars, reference holes remained in each slide.

Photographic slides of the colored sections were taken through a microscope (Zeiss Tessovar, camera: Contax). These were projected on a table through a slide projector (Kindermann AV100, lens: Isco-Göttingen Projar 1:2.8/60). The reference holes of the first slide were drawn on the table; those of the other projections were fitted on these drawings. In this way, magnification of each slide was the same. The outlines of the 4 muscles, their aponeuroses and the reference holes of each section were drawn on natural tracing paper. The sections were thoroughly examined under a microscope and relevant histological aspects that could not be seen properly in the projection were added to the drawings, using a drawing prism (Zeiss). Finally, 80 detailed drawings of the triceps surae muscle outline, including aponeuroses, tendons and large structures of louse connective tissue were obtained.

The outlines of the 4 different muscles, their aponeuroses and the 5 reference holes were digitized separately, using a xy-tablet (Summagraphics ID). Using reference hole coordinates, slide numbers and the two-dimensional 
registration of outlines in each plane, a three-dimensional reconstruction of the muscle complex was produced. The reconstruction was visualized by a computerized drawing program that allows separate drawing of selected outlines and presentation from any viewpoint or in any perspective (110).

\section{Finite element mesh generation}

After examination of the detailed reconstruction, 14 sections of the GCM, representing the total length of the muscle, were selected. These included the section containing the largest cross-sectional area of the GCM $(18.2 \mathrm{~mm}$ from the proximal origin), and those containing the most proximal part of the distal aponeurosis ( $5.8 \mathrm{~mm}$ from the proximal origin) and the most distal part of the proximal aponeurosis (16.2 $\mathrm{mm}$ from the proximal origin).

In order to generate three-dimensional elements for the finite element mesh, 28 nodes were indicated in each section and interconnected as follows (Figure 5.1). First, two nodes were placed on the muscle's outline, indicating its largest diameter $\left({ }^{*}\right)$. Then, two points $\left({ }^{\wedge}\right)$ were used to mark the edges of the aponeurosis on the outline, to eventually locate the aponeuroses in the mesh. If another aponeurosis was visible in the same slide, two more marks were used. Thereafter, 14 (or 12 in case of two aponeuroses) points were evenly distributed along the outline of the muscle fiber tissue. Care was taken to indicate the points in such a way that connective lines between the nodes followed the outlines of the muscle accurately. Using these 18 points, representing the muscle's outline, the muscle area was divided into $18\left(3^{\star} 6\right)$, approximately equally sized quadrangles with 28 nodes (Figure 5.1). They were fed into the computer, using the digitizer tablet. Corresponding nodes between successive sections were connected. This resulted in $13^{*} 3^{*} 6$ hexahedral elements, each of which was divided into 5 tetrahedral elements.

The aponeuroses were represented by two-dimensional triangular elements. These were attached to the sideplates of the tetrahedral elements at the muscle surface where aponeurosis was indicated in the cross-

\section{Figure 5.1}

Two marks ( $)$ on the muscle outline indicate its largest diameter, while 4 nodes (1) mark the aponeuroses widths. The aponeurosis thickness is exaggerated in the figure to clearly show its location. The 13 remaining nodes are evenly distributed along the muscle outline. A 47-grid results after interconnecting the nodes:

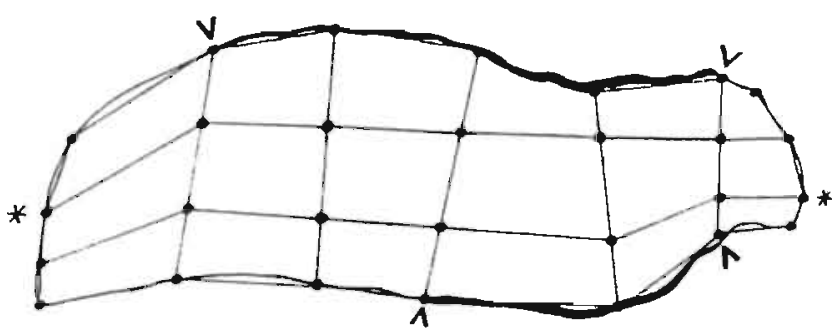


sections. Local aponeurosis thickness, measured from the digitizer tablet, was assigned to corresponding elements. A total of 54 and 76 triangular elements for the proximal and distal aponeurosis respectively, described the aponeuroses. Thus, a finite element mesh of the GCM, consisting of $5^{\star} 13^{\star} 3^{\star} 6 \quad(=1170)$ three-dimensional tetrahedral elements (muscle fiber tissue), 130 two-dimensional triangular elements (aponeuroses) and 392 nodes, resulted.

\section{RESULTS}

\section{Triceps surae muscle reconstruction}

Figure 5.2 and Figure 5.3 show the result of the detailed reconstruction. The names of the muscles are given in the figures. The dorsal (Figure 5.2A) and ventral view (Figure 5.2B) of the triceps surae muscle show the orientations of the different muscles. Proximally, the PL can be seen at the surface of the triceps surae muscle (Figure 5.2A and Figure 5.3A), while the remaining part is covered by the CGM and the GCL. The distal aponeuroses of the 4 muscles join distally, forming the Achilles tendon.

From the cross-sections (Figure 5.3) it is clear that the GCM only has one distal and the proximal aponeurosis, whereas the GCL contains two distal and two proximal aponeuroses. One of the distal aponeuroses divides the GCL into a lateral and a medial part (Figure 5.3B). The medial part is more pronounced distally, and shares a proximal aponeurosis with the PL. The GCM and GCL are interconnected distally, where they share an aponeurosis. Figure 5.3B shows an invagination of the distal aponeurusis

Figure 5.2

Dorsal $(A)$ and ventral $(B)$ view of the triceps surae muscle. Ordered from dark to light the figure shows the medial gastrocnemius (GCM), soleus (SOL), lateral gastrocnemius (GCL) and plantaris ( $P L$ ) muscle. Aponeuroses and the Achilles tendon are white.

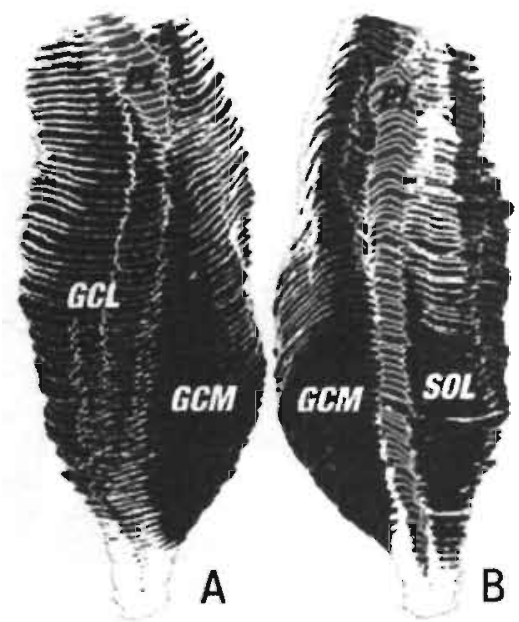


into the GCM dorsal. Such invaginations are also present in other aponeuroses (Figure 5.3C). Figure 5.2B shows the large neural-vascular bundle, accompanied by loose connective tissue, in between the GCL and the PL. This structure extends towards the GCM, while it remains located between the PL and the medial part of the GCL.

The place where the neural-vascular bundle enters the GCM can be identified on the ventrolateral view of the GCM (Figure 5.4A), near the insertion at the femur. The distal aponeurosis of the GCM (Figure 5.4A) encloses $62 \%$ of the length of the muscle, while the proximal aponeurosis (Figure 5.4B) is 78\% of the length of the GCM. Their thickness ranges from $0.02 \mathrm{~mm}$ to $0.56 \mathrm{~mm}$. The complete length of the GCM is $26.2 \mathrm{~mm}$ from the most proximal attachment to the bone to the most distal section where muscle fibers can be seen in the cross-sections. Both a cross-section and a longitudinal section in the ventromedial to dorsolateral plane through the GCM show the concavity of the muscle. The maximum diameter of the muscle in a cross-section is $10.0 \mathrm{~mm}$ in the ventromedial to dorsolateral direction, and in the perpendicular direction $4.2 \mathrm{~mm}$ (Figure 5.3B).

\section{Finite element mesh generation}

The final GCM mesh, displayed with Femview (Femview Limited, Leicester, LE2 1GF, England), is shown in Figure 5.4C. Viewpoints in Figure $5.4 \mathrm{~B}$ and Figure $5.4 \mathrm{C}$ are similar. The Achilles tendon starts at the lower end of the mesh. The muscle consists of 1170 tetrahedral elements for the muscle geometry, which can be identified in Figure $5.4 \mathrm{C}$, and 54 and 76 triangular elements for the proximal and the distal aponeurosis respectively. These triangular elements are attached to the triangular

Figure 5.3

Proximal (A), medial (B) and distal (C) cross-section through the triceps surae muscle. Ordered from dark to light the figure shows the medial gastrocnemius (GCM), soleus (SOL), lateral gastrocnemius (GCL) and plantaris (PL) muscle. Aponeuroses and the Achilles tendion are white.

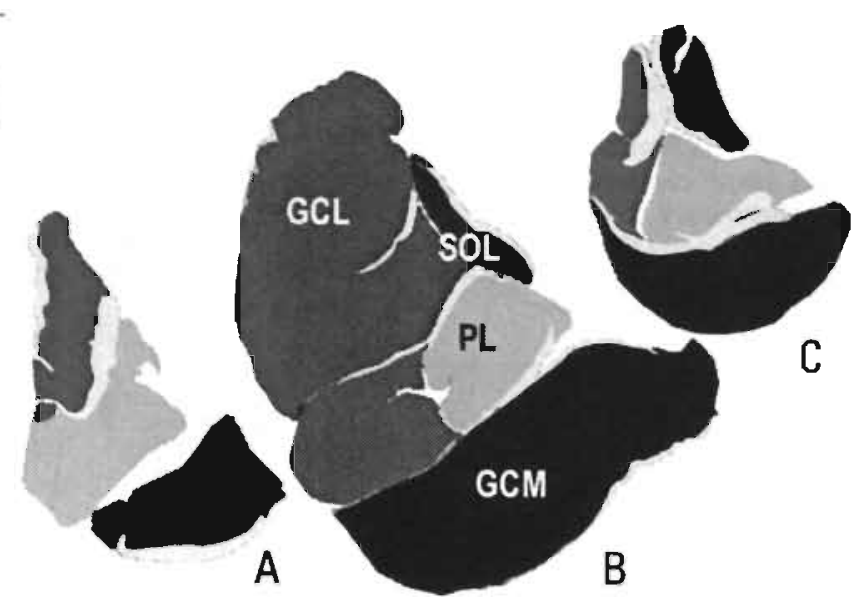


sideplanes of the tetrahedral elements, but cannot be identified in the figure. The gray levels in Figure $5.4 \mathrm{C}$ are a result of three-dimensional shading. Despite the large number of elements, the mesh contains no more than 392 nodes, resulting in acceptable calculation times for finite element simulations.

\section{DISCUSSION}

Triceps surae muscle reconstruction

The perfusion fixation of the muscle was established under well-controlled conditions. During the fixation the angle of the knee and the ankle were both held at 90 degrees to minimize deformation of the muscle as a result of the perfusion fixation. This was confirmed by X-ray images. After complete fixation, the muscle was carefully dissected free, dehydrated and embedded in plastic. Care was taken not to deform the muscle during these procedures. The next steps in the reconstruction included the production and the photographing of the cross-sections and the drawings of the slide
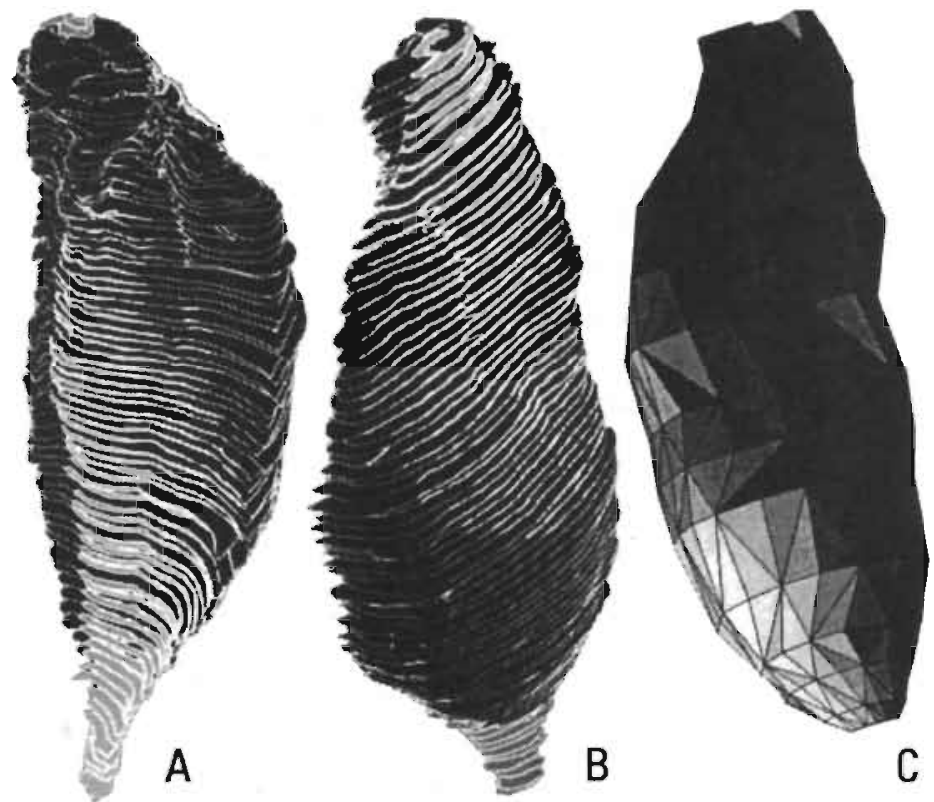

Figure 5.4

Ventrolateral $(A)$ and dorsomedial $(B)$ view of the detailed neconstruction of the medial gastrocnemius muscle. The thick white lines represent the aponeuroses. Viewpoint of the resulting finite element mesh $(C)$ is similar to the dorsomedial view (B). The gray values in (C) are a result of three-dimensional shading. 
projections on the tracing paper. Since the reference holes on the drawings fit to each other properly, it can be concluded that errors, resulting from the projections or the production of the sections, are minor. The resulting drawings at this point include the rough muscle outlines and the large collagen structures. To avoid projection errors using the drawing prism, structures are always centered under the prism and fitted to the structures that were drawn on the sheet.

Invaginations of small aponeuroses parts affect muscle fiber attachment in two ways. First, more fibers can attach, since the superficial side as well as the deeper side of aponeuroses can be used. Second, the angle of attachment of the fibers on the deeper side, which tends to decrease near the thinner part of the aponeurosis (109), is increased.

Because of tissue-shrinkage during the fixation of the muscle and the production of the sections, gaps occurred at some places in the intra- and intermuscular aponeuroses. Therefore, it is possible that the thickness of these aponeuroses was sometimes overestimated. Aponeuroses situated on the surface of the triceps surae muscle hardly show gaps.

\section{Finite element mesh generation}

Much morphological detail is included in an anatomical reconstruction of the rat triceps surae muscle. In the contrary, the number of nodes that could be used in the finite element mesh generation of the GCM was limited. To enclose as much detail as possible, characteristic locations of the muscle outline were marked. Special attention was paid to the location of the aponeuroses by indicating its transition to loose connective tissue in each section. All marks are included as nodes in the finite element mesh, which comprises the GCM geometry and its aponeuroses.

Local aponeurosis thickness is measured for each relevant node using a microscope. At approximately $80 \%$ of the nodes, aponeurosis thickness could be measured properly. At the remaining nodes, mainly located in the proximal aponeurosis, thickness is overestimated as a result of aforementioned artifacts in the histologic slides. When necessary, local thickness was estimated from the thickness at surrounding nodal points.

A finite element simulation of passive muscle perfusion is performed as an illustration of the use of the reconstruction by Vankan et al (109). Since the GCM, GCL and the PL are attached to each other and because of the muscle's specific geometry, analysis of detormation of the separate GCM during contraction is questionable. Suppression of deformation of the ventrolateral side of the muscle would probably better resemble deformation during contraction than allowing free surface deformation in finite element simulations. 
Finally, although muscle fibre directions are important for the behavior of the muscle during contractions, they have not been determined in this reconstruction for great experimental difficulties. Diffusion weighted MRI may be a suitable means to add fibre direction information in future studies (106).

\section{CONCLUSIONS}

We were able to make a detailed histologic reconstruction of the geometry of the triceps surae muscle, including muscle geometry, aponeuroses, tendons and large bundles of connective tissue. From this detailed reconstruction, a finite element mesh of the GCM was derived, including muscle geometry and the two aponeuroses of the GCM. This mesh can be used in simulations with the finite element model of perfused contracting skeletal muscle, which is formulated by our group, to calculate deformation, intramuscular pressure and blood pressure distribution during contraction. 

C.C. van Donkelaar, L.J.G. Kretzers,

F.H.M. Bovendeerd, L.M..4. Lataster,

K. Nicolay, J.D. Janssen, M.R. Drust,

Diffusion tensor imaging in biomechanical studies of skeletal muscle function, Journal of Anatomy, 194, 79-88, 1909 .

(Copyright 1998, Cambridge University

Pressi).

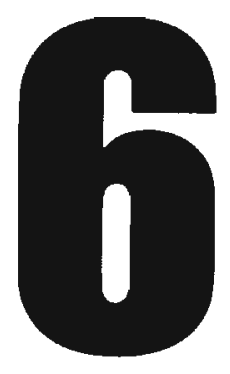

\section{DIFFUSION TENSOR IMAGING IN BIOMECHANICAL STUDIES OF SKELETAL MUSCLE FUNCTION}

Including muscle fiber directions is important for accurate simulation of muscle contraction. It is studied whether the first principal direction of diffusion, determined with DTI, coincides with the muscle fiber direction, and if the'se data are suitable to be used in numerical studies to skeletal muscle mechanics. 


\section{INTRODUCTION}

Biomechanical studies have shown that the mechanical behavior of muscle depends strongly on muscle architecture $(18,19,107)$. To analyze muscle mechanics and to calculate the distribution of mechanical properties such as stress and strain, accurate data regarding muscle fiber orientation are needed.

Traditional anatomical reconstruction techniques to determine threedimensional muscle fiber orientations have many disadvantages (64). Two alternative methods, based on MRI, have been used previously: highresolution (conventional) MRI and Diffusion Tensor Imaging (DTI). In high-resolution MRI of skeletal muscle, a striation pattern is visible provided the image is coplanar with the fascicle orientation (29). Complete three-dimensional reconstruction of fiber fields seems possible with this method (88), but a large number of planes is needed and fiber directions have to be determined from the images. DTI measurements are independent of the orientation of the image plane, and three-dimensional fiber directions are obtained immediately.

The DTI technique is based on measurement of the apparent diffusion of water in a (biological) tissue. From a series of diffusion weighted images, employing diffusion sensitization in six independent directions, a diffusion tensor can be calculated in each voxel (9). The eigenvalues and eigenvectors of this tensor provide information about local tissue anisotropy. The eigenvector belonging to the largest eigenvalue of the diffusion tensor is assumed to coincide with the local muscle fiber direction in striated muscle $(10,32,45)$ and with the myelinated fiber direction in brain white matter $(26,67,73,81)$. In the present paper, the direction of this eigenvector will be referred to as the DTI direction.

Qualitative resemblance between DTI directions and visual anatomy (10, $25,106)$ or essential features of cardiac architecture $(32,45,84)$ have been described in the literature. Basser showed that the principal directions of diffusion co-rotated with the orientation of a meat sample in successive measurements (10). This demonstrates that they are intrinsic to the material, but not that they coincide with fiber directions. The only study with respect to the quantitative accuracy of the DTI technique attended cardiac muscle. DTI fiber orientation and histological slices showed distinctive correlation (45). It is hypothesized that this holds for skeletal muscle as well as for cardiac muscle.

The first aim of the present study is to verify the hypothesis that DTI fiber orientation coincides with skeletal muscle fiber direction. Therefore, DTI directions in the midsagittal plane of the tibialis anterior muscle of a rat 
were compared qualitatively with fascicle orientations that were visible in high-resolution MR images, and quantitatively with fiber directions measured in an actual longitudinal section through the same plane of that muscle. In addition, this study shows the practical use of the DTI method in biomechanical research to skeletal muscle functioning. The finite element (FE) method, which has become familiar in biomechanical research on skeletal and cardiac muscle behavior $(18,51,116,117)$, essentially makes use of geometric and spatial information. One major difficulty and timeconsuming issue in such studies is to generate accurate FE meshes. Furthermore, in FE models of contracting skeletal muscle not only is the muscle geometry important, but simulations are also very sensitive to fiber directions. They may become unstable with large variations in fiber direction (too small voxel size) but become inappropriate if the fiber direction input is erroneous, for instance if the $\mathrm{V}$-shaped fiber direction around an intramuscular aponeurosis is smoothed (too large voxel size).

The combination of high-resolution MRI and DTI provides a potential way of simplifying the creation of FE meshes for any kind of skeletal muscle. In the present study, the TA muscle contour is determined from a highresolution MR image and an appropriate voxel size from the DTI data, to incorporate DTI-determined muscle fiber directions in a FE model of contracting skeletal muscle. This was done by clustering original voxels to larger effective voxel sizes. A FE simulation of a tetanic contraction will illustrate the results.

\section{MATERIAL AND METHODS}

The left hind leg of an anaesthetized male Lewis rat (age 11 weeks) was fixed at an ankle angle of $110^{\circ}$ (slight plantar flexion) and a knee angle of $90^{\circ}$. After perfusion fixation with $5 \%$ buffered formalin, the left leg was dissected and the skin and part of the dorsal musculature were removed. The leg was fixed in a PVC tube (outer diameter $20 \mathrm{~mm}$, inner diameter $17 \mathrm{~mm}$ ) using polyurethane foam. A groove (depth $0.8 \mathrm{~mm}$, width $1.0 \mathrm{~mm}$ ) with an increasing pitch was drilled in the outer surface of the tube and filled with ECG electrode gel. As this groove was visible in MR images and in the specimen, it served as an external reference system to link results. Two glass hematocrit capillaries (inner diameter $0.22 \mathrm{~mm}$ ) were filled with tap water and glued to the outside of the tube at both sides of the estimated position of the midsagittal TA plane. In combination with MR images, these capillaries facilitated determination of the midsagittal TA plane.

MR images were obtained at $20^{\circ} \mathrm{C}$ with a $4.7 \mathrm{~T}$ SISCO $200 / 400$ system, operating at $200 \mathrm{MHz}$ for protons and equipped with Oxford gradients (maximal gradient strength of $220 \mathrm{mT} / \mathrm{m}$ ). On the basis of high-resolution images, it was ensured that the $x, y$ and $z$ axes of the magnet coincided 
with the longitudinal (proximodistal), transverse (lateromedial), and sagittal (dorsoventral) muscle axes, respectively.

Diffusion was measured by incorporating pulsed gradients into a spin echo imaging sequence, according to Stejskal \& Tanner $(81,99)$. Pulsed gradients were placed symmetrically around the $180^{\circ}$ refocusing pulse. The readout gradient rephasing lobe was placed directly before the echo acquisition. Gradient strength was increased from 0 to $117 \mathrm{mT} / \mathrm{m}$ in 3 steps (i.e. 4 gradient strengths were applied), in each of the 6 directions: $x, y, z, x y, x z$ and yz. Echo time equaled $35 \mathrm{~ms}$, diffusion gradient pulse duration and separation were 7.5 and $15 \mathrm{~ms}$ respectively (106).

For each voxel, an apparent diffusion coefficient in each of the 6 independent directions $(x, y, z, x y, x z$ and $y z)$ was calculated from the measured signal intensities at 4 applied gradient strengths. Values based on a correlation between the natural logarithm of the relative signal intensity and the b-value of less than 0.9 were excluded from further analysis. From the 6 diffusion coefficients, 1 for each of the 6 directions, diffusion tensors were obtained from which eigenvalues and eigenvectors per voxel were calculated. The eigenvalues were sorted in decreasing magnitude: $\lambda_{1}>\lambda_{2}>\lambda_{3}$. The eigenvector belonging to $\lambda_{1}$ is thought to represent the local muscle fiber direction and will therefore be referred to as DTI direction. The above procedure has been described in more detail by Basser et al (9).

High-resolution MR images of sagittal (HRS) and transverse (HRT) muscle planes ( $x-z$ and $y-z$ plane of the MRI magnet), as well as DTI data of sagittal planes were obtained. DTI and HRS images were collected in 15 contiguous slices of $0.6 \mathrm{~mm}$, in a field of view of $70^{\star} 35 \mathrm{~mm}^{2}$. There was $1.4 \mathrm{~mm}$ distance between the 15 successive $0.6 \mathrm{~mm}$ thick HRT images, in a field of view of $35^{\star} 35 \mathrm{~mm}^{2}$. Data matrix of HRS and HRT measurements was $256^{\star} 256$, whereas DTI images consisted of $128^{*} 128$ pixels. Thus, DTI voxel size $\left(x^{*} y^{*} z\right.$ ) equaled $0.55^{*} 0.6^{*} 0.27=0.090 \mathrm{~mm}^{3}$. The positions of all DTI and HRS planes are indicated in the HRT image through the thickest part of the TA muscle, as shown in Figure 6.1.

After the MRI experiment, the midsagittal plane of the specimen was found to coincide with slice 9 (Figure 6.1), as determined from the external reference system and all high-resolution images. The midsagittal plane of the muscle is defined as the plane parallel to the sagittal plane of the body that includes the thickest part of the TA (see Figure 6.1). The images that correspond to this plane will be referred to as HRS_9 (the midsagittal highresolution image) and DTI_9 (the DTI image of the same plane). The specimen was sawn through this specific plane, using an Exakt diamondcoated band saw with a D64 sawing blade. Due to machining, the opposing surfaces in the sawing plane were approximately $0.4 \mathrm{~mm}$ apart. The medial 
surface of the lateral half of the specimen resembled the HRS_9 image. This surface will be referred to as ALS (actual longitudinal section).

Surface contrast of ALS was enhanced by 15 seconds staining with $0.2 \%$ toluidine blue. Muscle fibers were clearly visible on the exposed surfaces, except for a small area ventral to the distal aponeurosis. A photographic slide (Ektachrome 64) was made with a Nikon F501 camera and a micro Nikkor 105-mm lens. The contours of the TA muscle, including its aponeuroses, as well as the positions of the reference frame, were traced from an $80^{\star} 20 \mathrm{~cm}^{2}$ projection with a Zeiss Unimat projector and a Zett Varioo Talon lens. A computer slide of HRS_9 was projected and traced in the same way. These tracings served as a basis to couple the frames of reference from ALS and MRI, to permit comparison of the fiber fields. ALS fiber angles on specific locations were determined from the projected slide, using a digitizer table (Summagraphics ID).

HRS_9, DTI_9 and ALS all provide information regarding local fiber directions. The fiber angle was defined as the angle between DTI or muscle fiber direction and the positive $x$-direction (see Figure 6.3 for the orientation of the axes). DTI_9 was qualitatively compared with HRS_9 by projecting unity vectors of the first eigenvectors of DTI_9 on that image. Quantitative comparison between ALS and DTI_9 was performed in two ways. Firstly, a distributed region of interest was defined as 29 positions within the TA, including the complete range of fiber directions in the ALS. As the fiber direction was not clearly visible in ALS in the area ventral from the distal aponeurosis, this region was excluded. The local ALS fiber directions in the distributed region of interest were measured. The error

\section{Figure 6.1}

Transwerse high-resolution image (HRT_8) halfway through the TA (orientation: left. ventral, bottom: lateral). The $T A$, roughly indicated by a black polygon, can clearly be seen just below the white, semi-circular tibia in the left upper part of the picture, Locations of the 15 HRS and the 15 DT7 slices are indicated.

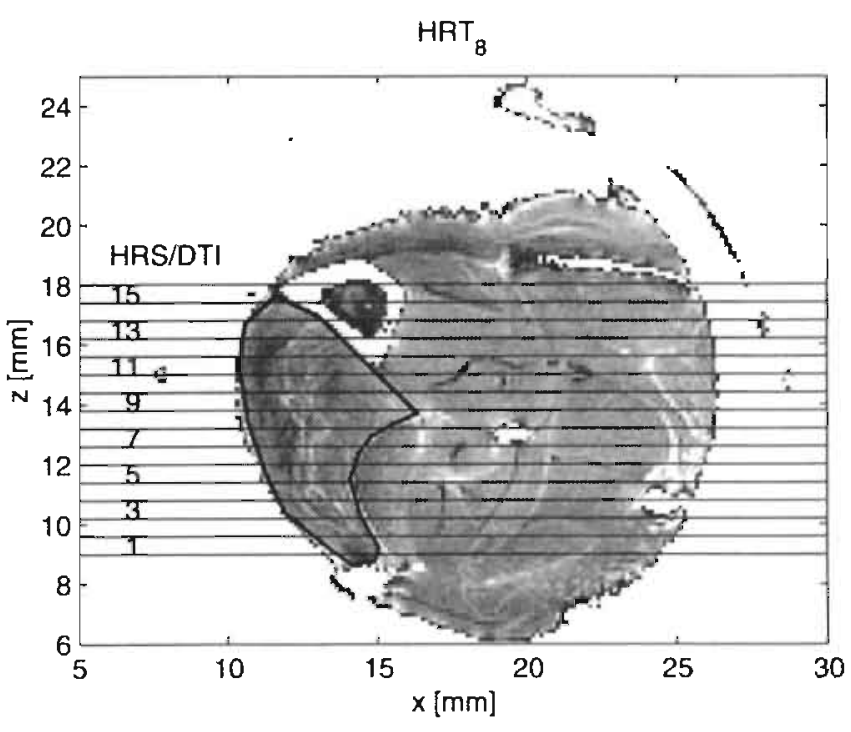


angle, defined as the angle between ALS and DTI_9, was calculated for each of the 29 locations. Secondly, another region of interest was defined in the $\mathrm{TA}$, this being the proximal area in which muscle fibers run predominantly parallel. The average ALS fiber direction was measured, and the variation in fiber angles in this area was estimated from the ALS projection. In DTI, the proximal region of interest is the area between $(39.7,9.3)$ and $(47.9,13.4)$ in Figure 6.3A and contains $225\left(15^{\star} 15\right)$ DTI voxels. The mean DTI angle and its standard deviation were compared with the general ALS fiber direction in this area.

An appropriate voxel size for the use of DTI muscle fiber directions in FE models was defined as the smallest voxel size that provides a standard deviation of the fiber directions of $<5^{\circ}$. To determine an appropriate DTI voxel size, new effective voxels of different sizes were defined within the previously mentioned proximal region of interest. These new voxels consisted of at least 4 voxels, the ratio of their sides being in between 0.5 and 2 voxels. A new diffusion coefficient for each of the six directions was calculated per new voxel, this being the average diffusion coefficient of the original voxels within the new one. Averaging diffusion coefficients is identical to averaging the primary image data, since those are linearly related. From the averaged values, new DTI directions were calculated. The mean DTI direction and standard deviation of the new voxels within the proximal region of interest were calculated for each effective voxel size. Theoretically, the standard deviation would decrease by $n^{-0.5}$, where $n$ is the number of original voxels per new voxel, whereas the mean DTI direction should not change.

To create the FE mesh to be used in the simulation of a muscle contraction, TA contours on the HRS_9 image were obtained by marking outline coordinates on the high-resolution image in conjunction with close examination of the ALS under a stereomicroscope. From this contour, the program Sepview (Sepra Analysis, Leidschendam, The Netherlands) automatically generated the distribution of elements in this particular geometry. A local fiber direction was assigned to each element, which was calculated by averaging the DTI directions in the previously determined appropriate voxel size surrounding the element center. A simulated tetanic contraction of the TA with these realistic fiber directions was performed using the finite element model of perfused contracting skeletal muscle recently described by Vankan et al(113-117).

\section{RESULTS}

The diffusion images of DTI_9 in the 6 directions $x, y, z, x y, x z$ and $y z$ are shown in Figure 6.2. Judging from the intensity of the diffusion image in the $x$-direction, diffusion of water is casiest in that direction. 
The HRS_9 image is shown in Figure 6.3A. The TA covers the area ventral to $\mathrm{z}=15 \mathrm{~mm}$ almost completely. Its distal aponeurosis is clearly visible in this image as a dark line from coordinates $(26,13)$ to $(36,11)$. Muscle fascicles are visible as faint striation over approximately half of the muscle, dorsally less clear than ventrally. Note the pennate insertion of the fascicles on the distal aponeurosis.

In Figure 6.3B, a projection of DTI_9 directions is superimposed on HRS_9. The DTI direction in each voxel in this image is based on averaged diffusion coefficients over 10 surrounding voxels. This image is an enlargement of the area around the distal aponeurosis in Figure 6.3A. Since the lines are two-dimensional projections of three-dimensional unit vectors, shorter lengths imply larger out-of-plane directions. The DTI directions follow the muscle fascicle direction visible as faint striation in HRS_9 quite well, and the pennate insertion of DTI directions on the distal aponeurosis is obvious. The outermost DTI directions along the ventral side of the TA

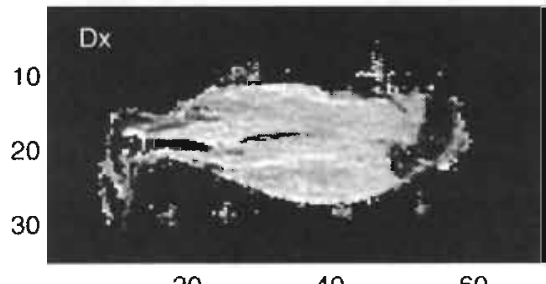

20

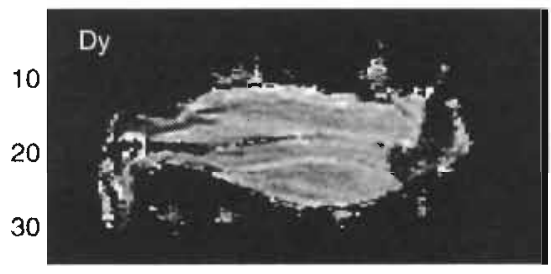

20

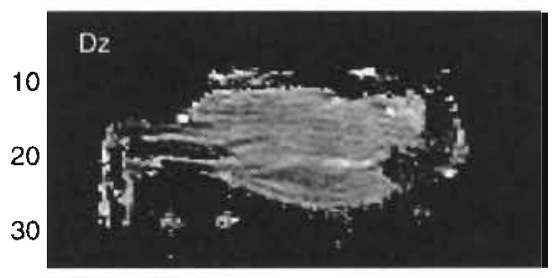

20

$$
40
$$

60
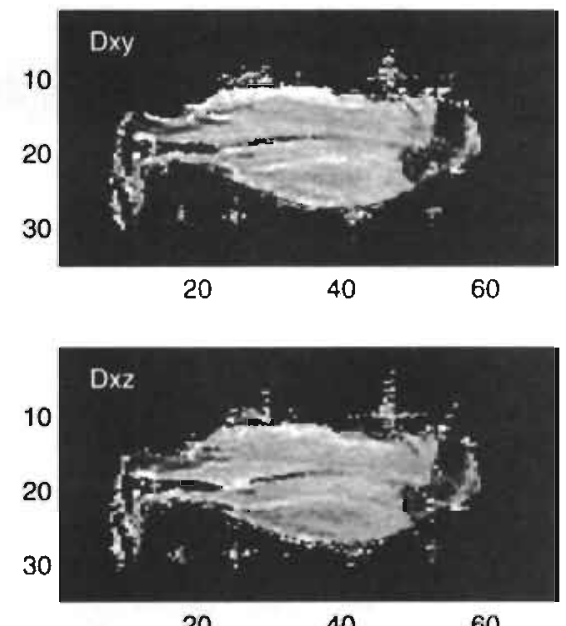

20

40

60

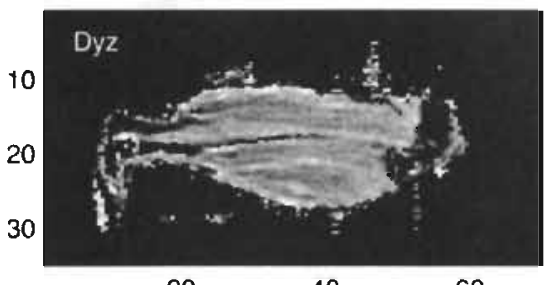

20

60

Figure 6.2

Diffusion maps of the 6 directions $D x, D y, D z, D x y, D x z$ and $D y z$ of DTI 9. The corresponding high-resolution image is shown in Figure 6.3. Differences in pixel intensity between Dx, i.e. the expected predominant fiber direction, and $D y / D z$, perpendicular to the fiber direction are obvious. 
are less ordered and do not follow the expected fiber direction. These voxels only partly contain muscle tissue, resulting in a lower signal to noise ratio in the original diffusion weighted images.

Table 6.1 shows the means and standard deviations of the 3 principal directions, the ratio between $\lambda_{1}$ and $\lambda_{3}$ and the trace of the diffusion tensor for the voxels in the distributed and the proximal region of interest. The large $\lambda_{1}$ with respect to both $\lambda_{2}$ and $\lambda_{3}$ reflects the strong transverse isotropic character of the tissue. The values correspond to those found in the literature (see Table 6.1), although our ratio $\lambda_{1} / \lambda_{3}$ is somewhat higher than that found in other studies.
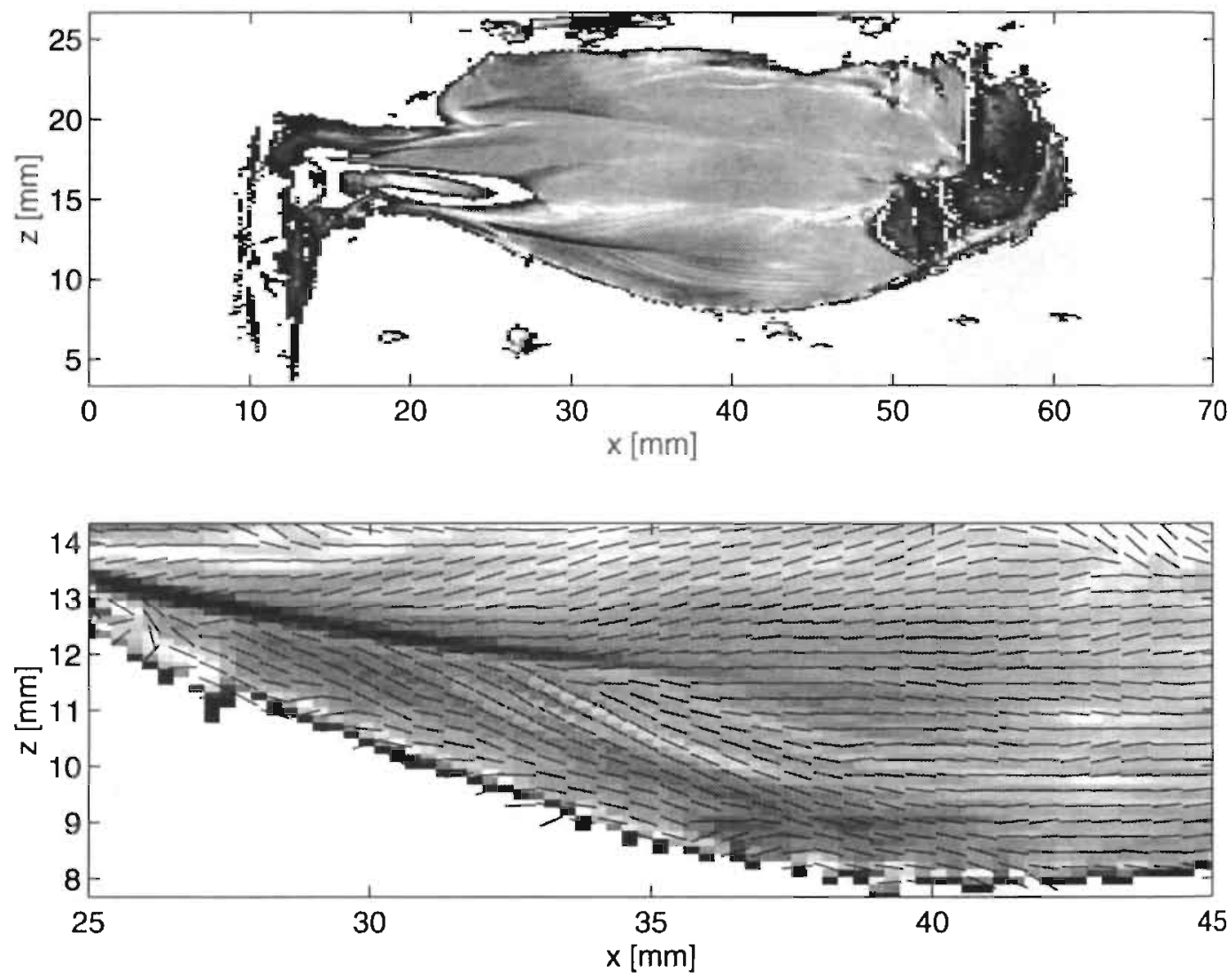

Figure 6.3

A. High-resolution image HRS 9 through the midsagittal plane of the TA (orientation: left: distal, bottom: ventrali). Note the pennate insertion of the muscle fascicles (faint striation) on the dark distal aponeurosis $((26,13)$ to $(36 ; 11))$.

B. Enlargement of HRS_9 of the area around the aponeurosis, with a superimposed projection of DII 9 directions (direction in each voxel based on averaged apparent diffusions of 10 voxels). 
The mean error angles and standard deviations between ALS and DTI_9 in the distributed region of interest are given in Table 6.2 for the original data and after averaging the diffusion coefficient over different numbers of voxels. At larger effective voxel sizes, error angles are significantly different from 0 ( $p<0.05)$.

In the proximal region of interest, the general ALS angle equals $0.3^{\circ}$, with an estimated variation due to a small gradient in this area of $2^{\circ}$. For each of

Table 6.1

The three principle diffusion coefficients and quantities derived from these data, in the distributed (per cluster of voxels) and in the proximal region of interest. Values (mean $\pm \mathrm{sd}$ ) in $10^{\circ} \mathrm{m}^{2} \mathrm{~s}^{l}$. These data are compared with those found in other studies. Van Doorn et al. (106): measurement of formalin fixated cat semimembranosus muscle at $20^{\circ} \mathrm{C}$; Basser et al. (10): measurement of pork loin at $15^{\circ} \mathrm{C}$ (left column) and $15.5^{\circ} \mathrm{C}$ (right column); Cleveland et al. (25): nonfixated TA of mature male rat, 10 min to 24 hours after dissection, $25^{\circ} \mathrm{C}$

\begin{tabular}{rlllllll}
\hline & $\begin{array}{l}\text { Distributed } \\
\text { ROI }\end{array}$ & $\begin{array}{l}\text { Proximal } \\
\text { ROI }\end{array}$ & $\begin{array}{l}\text { Hsu et al. } \\
(45)\end{array}$ & $\begin{array}{l}\text { Van Doorn } \\
\text { et al. (106) }\end{array}$ & $\begin{array}{l}\text { Basser } \\
\text { et al. (10) }\end{array}$ & $\begin{array}{l}\text { Cleveland } \\
\text { et al. (25) }\end{array}$ \\
\hline$\lambda .1$ & $1.25 \pm 0.08$ & $1.17 \pm 0.38$ & $0.94 \pm 0.28$ & $1.37 \pm 0.31$ & 1.078 & 1.053 & 1.39 \\
$\lambda 2$ & $0.91 \pm 0.10$ & $0.84 \pm 0.27$ & $0.74 \pm 0.27$ & & 0.949 & 0.948 & \\
$\lambda 3$ & $0.75 \pm 0.09$ & $0.70 \pm 0.23$ & $0.63 \pm 0.24$ & $0.93 \pm 0.31$ & 0.836 & 0.893 & 1.0 \\
$\lambda 1 / \lambda 3$ & 1.67 & 1.67 & 1.49 & 1.47 & 1.29 & 1.18 & 1.39 \\
Trace & 2.91 & 2.71 & 2.31 & & 2.836 & 2.894 & \\
\hline
\end{tabular}

Figure 6.4

Standard deviations of DT angles $\alpha\left(^{\circ}\right)$ with different effective voxel sizes $s$ $(\mathrm{mm})$ in the proximal region of interest. The solid line represents the best fit through these data, using the function $s d=c^{*} s^{05}$, in which $c=4.76^{\circ} \mathrm{mm} \mathrm{m}^{0.5}$. Unlike the mean angle $(-1.39+0.99)^{\circ}$, the standard deviation strongly depends on voxe/ size.

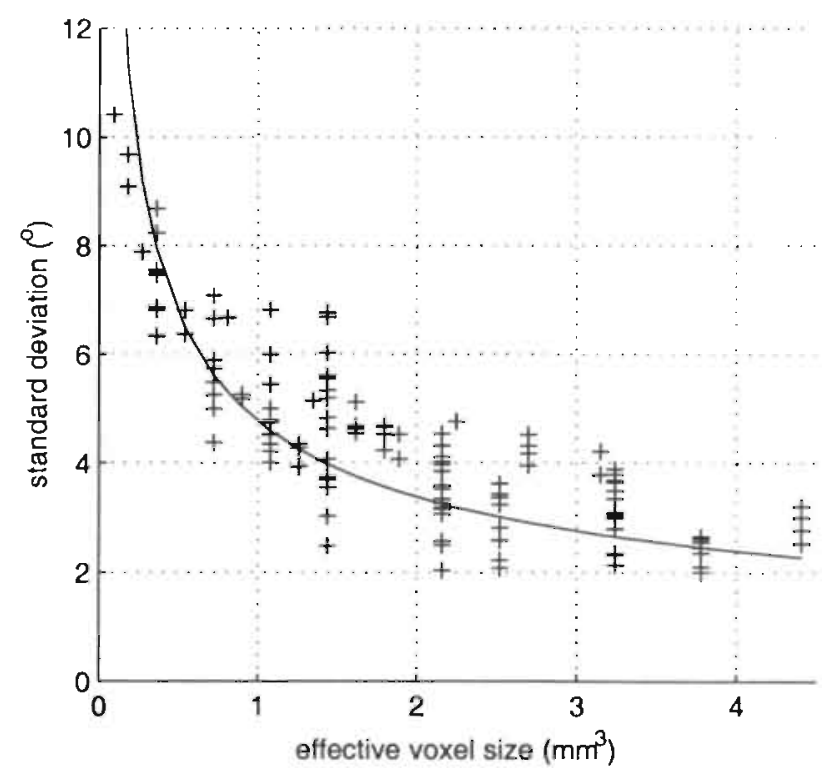


the 125 possible combinations of effective voxel sizes in this area, a mean DTI direction was calculated. The mean value of these 125 mean DTI directions equals $-1.39^{\circ} \pm 0.99^{\circ}$. Thus ALS and DTI in the proximal region of interest differ by $\sim 1.7^{\circ}$. The 125 standard deviations of these effective voxel sizes are essential in the determination of an appropriate voxel size for the FE mesh. They are plotted as a function of effective voxel size in Figure 6.4. A least squares fit for the hypothetical decrease of the standard deviation of DTI direction $(\alpha)$ with the square root of the voxel size (s) yielded $\mathrm{sd}(\alpha)=\mathrm{c}^{\star} \mathrm{s}^{-0.5}$, with $\mathrm{c}=4.76^{\circ \star} \mathrm{mm}^{3 / 2}(\mathrm{r}=0.86)$.

The FE mesh that was created is projected over the HRS_9 image in Figure 6.5A. In Figure 6.5B, the fiber direction as determined from the appropriate voxel size is shown per element. Proximally and distally thick lines indicate the sites where the muscle is fixed to the tibia and the first metacarpal bone. The corresponding nodes are fixed during the FE simulation. Material and contraction properties are similar to those used by Vankan et al (116) for the medial gastrocnemius muscle. An $800 \mathrm{~ms}$ tetanic contraction of $100 \mathrm{kPa}$ muscle tension is computed. Calculated deformation just prior to relaxation is shown in Figure 6.5C.

\section{DISCUSSION}

The high-resolution images show a high contrast between fat, bone, aponeurosis and muscle. A large part of HRS_9 shows muscle fascicle directions in the high-resolution image (Figure 6.3A). This is in agreement with the findings of Scott $e t$ al (88). The striking resemblance between the

\section{Table 6.2}

Means and standard deviations (") of the proximal and distributed region of interest for the original and averaged data. The values in the column with data from the distributed region of interest are actual mean and standard deviations of the difference in angles () between local DTI and ALS. The angles in the column for the DII data in the proximal region of interest were calculated from the formulae: mean $=0.03^{*}$ (voxel size) -1.45 and $s d=4.76{ }^{*}$ (vovel size) ${ }^{\text {th }}$ (see Figure 6.4). Thus these result from averaging different voxels. In case of significant differences between DTI and ALS, the significance $(p<0.01$ or $p<0.05)$ is added to the table.

\begin{tabular}{ll}
\hline & Distributed ROI (DTI-ALS) \\
\hline Original data $\left(0.09 \mathrm{~mm}^{3}\right)$ & $-2.49 \pm 10.48$ \\
Averaged 8 voxels $\left(0.72 \mathrm{~mm}^{3}\right)$ & $-3.16 \pm 5.28(\mathrm{p}<0.01)$ \\
Averaged 10 voxels $\left(0.90 \mathrm{~mm}^{3}\right)$ & $-1.11 \pm 3.12$ \\
Averaged 12 voxels $\left(1.08 \mathrm{~mm}^{3}\right)$ & $-1.90 \pm 3.14(\mathrm{p}<0.01)$ \\
Averaged 15 voxels $\left(1.35 \mathrm{~mm}^{3}\right)$ & $-1.54 \pm 3.16(p<0.05)$ \\
Averaged 25 voxels $\left(2.24 \mathrm{~mm}^{3}\right)$ & $-1.16 \pm 3.03(p<0.05)$ \\
\hline
\end{tabular}


projection of the DTI_9 fiber direction averaged over 10 voxels and the fascicle orientation in HRS_9 is evident (see Figure 6.3B). The same holds for other planes of the TA muscle. It can therefore be concluded that the qualitative agreement between DTI fiber directions and HRS fascicle orientations is very good.

The DTI directions in the proximal region of interest do not differ from the ALS fiber directions ( $p>0.1$ for all effective voxel sizes). However, in the distributed region of interest, DTI angles are significantly different from ALS angles $(p<0.05)$ for larger effective voxel sizes (Table 6.2). Probably, inplane gradients in fiber directions cannot be measured accurately with larger voxels. These gradients are present in the distributed region of interest, but not in the proximal one. More likely however, the approximately $1.7^{\circ}$ difference between DTI and ALS both in the proximal and the distributed regions of interest result from a misalignment between ALS and the DTI_9 slice. A slight misalignment was determined from the external reference marks due to loss of material during the sawing procedure. As a result, fiber angle gradients affect the comparison between ALS and DTI. This effect is strengthened by the fact that ALS is determined from the surface of the sawing plane, whereas DTI fiber directions are volume-averaged quantities. Other translations and rotations of ALS with respect to MR images have also been considered. A small $\left(<2^{\circ}\right)$ rotation

\section{Figure 6.5}

A. The finite element mesh, created on the basis of MRI experiments, plotted over the HRS 9 high-resolution image. $B$. The fiber directions per element were obtained from DTI data that were calculated from averaged diffusion tensors over 10 voxels. The elements that have no fiber direction indicated represent the proximal and distal aponeuroses. Those are irrelevant with respect to the fiber direction measurements.

C. The calculated deformed geometry of the TA muscle during a $100 \mathrm{kPa}$ isometric contraction.

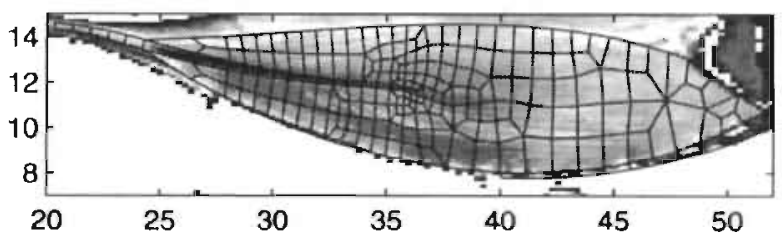

A

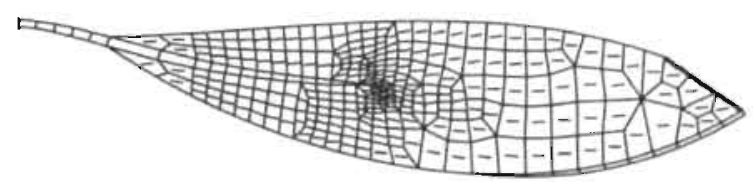

B

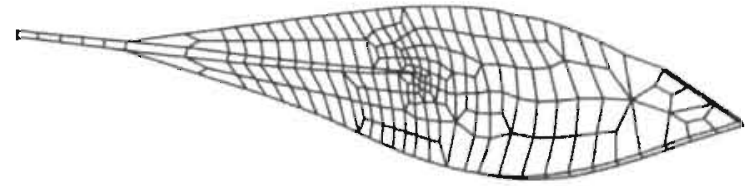


around the $z$ axis exists, which is calculated not to affect the final fiber direction measurements.

Based on the perfect agreement between HRS and DTI and in view of this discussion regarding differences between ALS and DTI, it can be concluded that DTI does indeed measure skeletal muscle fiber directions in fixated skeletal muscle.

The appropriate voxel size has been determined from the relationship between effective voxel size $s$ and standard deviation of DTI angle $\alpha$. For basic statistical considerations this relationship has been described using the function $s d(\alpha)=c^{*} s^{-0.5}$. Since $2^{\circ}$ variation in ALS is included in the measurement, the calculated relationship is a slight overestimation of the actual standard deviation. The voxel size with $5.0^{\circ}$ standard deviation equals $0.9 \mathrm{~mm}^{3}$, which equals 10 times the original voxel size (Figure 6.4). The fiber directions shown in Figure 6.3, based on this voxel size, nicely follow the HRS_9 fascicle directions. Regarding this procedure it should be kept in mind that accuracy increases but resolution decreases with larger voxel sizes. The ideal voxel size for measurements of fiber directions will therefore depend on actually present gradients in fiber direction and on the aim of the study. In the present data set, the strong fiber direction transition over the distal aponeurosis obviously remains. Even so, pennation angle on the distal aponeurosis is underestimated as a result of the averaging procedure.

The presented relationship between effective voxel size and DTI angle is obtained for formalin fixed skeletal muscle and depends on environmental variables such as temperature $\left(20^{\circ} \mathrm{C}\right.$ in our study) and the parameters of the MR experiment, notably the signal-to-noise ratio in the images. Therefore, the appropriate voxel size cannot be ported directly to other experiments. However, a criterion such as the one used in the present study can be used in all experimental conditions.

The obtained DTI muscle fiber directions are appropriate for inclusion in FE simulations of contracting skeletal muscle, as the presented simulation shows. The FE mesh that was used in this simulation was obtained from high-resolution MR images of the same muscle. This illustrates the potential of MR imaging techniques in biomechanical research on skeletal muscle function. This potential is even greater as the calculated deformation could in principal be combined with, for instance, tagging MRI

\section{CONCLUSION}

It is concluded that DTI directions actually represent local muscle fiber directions in the rat TA muscle. DTI fiber directions resemble fascicle 
directions visible in high-resolution images very well and specific features such as the pennate insertion on the distal aponeurosis are clearly present. The quantitative analysis indicates good analogies between DTI and ALS, although the correlation was not as obvious as was found for cardiac muscle (45). Possible reasons for this have been discussed.

DTI fiber directions can be used directly in numerical simulations of skeletal muscle contractions, provided that the variation in fiber directions is acceptable. An appropriate voxel size, providing standard deviations in angle below $5^{\circ}$, was determined for the current data set after the experiment $\left(0.9 \mathrm{~mm}^{3}\right)$. It was shown that from the combination of highresolution MR images and DTI measurements, accurate FE meshes could be generated. This shows the potential of MR techniques in the biomechanical research to skeletal muscle function. 



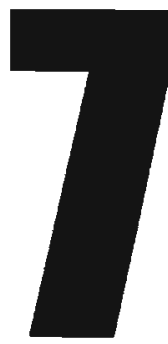

\section{DISCUSSION}

In a recapitulation of this thesis, the main idea behind the combination of experiments and a finite element model is evaluated. Further, a general discussion regarding experiments and the model points into directions for further research. Finally, the main conclusions that are drawn in the individual chapters of this thesis are summarized. 


\section{RECAPITULATION}

The main research objectives of this thesis are first to study the spatial interaction between contraction, intramuscular pressure and regional tissue perfusion, and second to determine three-dimensional muscle surface deformation during contraction. These topics are studied using a direct combination of animal experiments and a specially developed finite element model of perfused contracting skeletal muscle. The main advantage of this approach is that both experiments and simulations can be specially designed to come up with specific results, needed to support one another. These two methods are not used separately, but interconnect throughout the research, which provides possibilities to study topics that are unable to access from experiments or simulations only. The following paragraphs recapitulate the way experiments and simulations in this thesis are interwoven.

Chapter 2 shows how simulations enable interpretation of experimental data, while at the same time a comparison of experimental data with simulations points into directions to improve the model. Total muscle arterial and venous flows and muscle blood volume change are measured. The experimental data suggest that flow impediment results from volume change at the venous side, which is in agreement with the vascular waterfall phenomenon, but forceful conclusions could only be drawn in conjunction with numerical simulations. It is concluded that the impediment of perfusion during contraction is mainly caused by an initial vascular volume change in the venous compartment, whereas other compartments steadily decrease volume when contraction sustains. Although quantitatively flow signals between experiments and simulation do not agree, the simulation provides insight into muscle volume change. Quantitative differences between simulations and experiments come to the account of excessive volume change in the model. Suggestions for model improvement are provided in chapter 2.

Chapter 3 presents a numerical study to the vascular waterfall behavior during contractions. This waterfall phenomenon is widely studied using highly controlled experimental designs, which show a distinct interaction between tissue pressure rise and perfusion. However, geometrical effects, resulting from the distributed intramuscular pressure during contraction, cannot be studied in such experiments. As these can be explored by means of the presently used numerical model, this part of the thesis shows the value of model simulations to physiological research. A final comment on this chapter involves the wordings that are used. Like in the general introduction (chapter 1), effort was taken to allow experimental co-workers without engineering background to understand model in- and output as far 
as necessary to understand the study. Mathematical descriptions are avoided, whereas the text deals with venous blood pressure, flow from arteries to arterioles and capillary blood volume, rather than with pressure gradients and permeability tensors.

It was argued in the general introduction that spatial, multi-dimensional effects would be important to muscle functioning. Despite, chapter 2 and 3 only used two-dimensional plane strain models. Chapter 4 aims at determining the importance of three-dimensional effects with respect to mechanical muscle functioning by studying three-dimensional deformation. Therefore, three-dimensional displacements of markers, attached to the muscle surface, are calculated into longitudinal and transverse surface strain distributions. In the literature, muscle deformation is described from an anatomical point of view, using parameters like aponeurosis length change or pennation angle. The presently used strain parameters ease future comparison with finite element outcome. A comparison of strains between experiments and simulations is not captured by the present study, as the used two-dimensional mesh essentially lacks the muscle surface that is available for deformation measurements. For accurate strain calculation of the muscle surface during contraction, especially when effects of muscle length changes are studied, simulations must be performed with an anatomically realistic, three-dimensional mesh.

For the purpose of obtaining a three-dimensional finite element mesh, the rat calf muscle-tendon complex was reconstructed in three dimensions from histologic slides (chapter 5). Based on this reconstruction, a threedimensional mesh was generated that was actually used in finite element simulations by Vankan et al (113). However, simulations with this geometry and comparison of strain with the experimental findings of chapter 4, were not performed in the present study for a number of reasons. Accurate fiber directions are not available, which is essential for proper calculation of deformation. Further, the size of the mesh is too large to handle practically and simulations take too much computational time. Instead of the three-dimensional mesh, a two-dimensional representation of the midsagittal section of the medial gastrocnemius muscle was used in simulations (chapters 2 and 3).

The two main disadvantages of the method used in chapter 5 are that it is time-consuming and that it is difficult to obtain fiber directions from successive histologic slides. MRI techniques provide a solution to the'se problems (chapter 6). Three-dimensional skeletal muscle fiber directions of the rat anterior tibialis muscle are obtained with diffusion tensor imaging (DTI) and muscle contours are determined from high-resolution MRI measurements. A simulation with a two-dimensional finite element mesh including accurate fiber directions, based on these data, is included in 
chapter 6 . Chapters 5 and 6 are examples in which experiments support the finite element model by providing accurate model input data.

Summarized, the present thesis resides in between animal experiments and a sophisticated numerical model, in an effort to combine these two methods efficiently. It shows how experiments support models and models support experiments in order to increase the current understanding of skeletal muscle functioning. To allow proper comparison, experimental data are provided in mechanical terms that are suitable for comparison with finite element simulations, whereas model parameters and relationships are described in physiological terms rather than by numerical equations. This is found more suitable in the context of the present thesis, although previous experimental studies to skeletal muscle functioning often used parameters related to muscle anatomy, and the functioning and constitution of the model can be described more precisely with mathematical formulations. Where applicable, the text refers to the literature for detailed explanations of the model.

\section{DISCUSSION AND RECOMMENDATIONS}

\section{General}

In this thesis, four chapters include experiments and three include simulations. Chapter 5 and 6 aim at creating finite element meshes from geometrical data, obtained from dead muscle tissue. An example of a finite element simulation with the obtained mesh is provided in chapter 6 . Chapter 3 describes simulations of spatial blood perfusion during muscle contractions without bothering experiments. In chapters 2 and 4 flow and deformation during actual muscle contractions are experimentally determined. The closest evaluation of the accuracy of simulations is performed in chapter 2, where muscle blood flows are compared between simulations and experimental data. A better model validation would have been obtained by comparing the spatial distribution of relevant mechanical variables in the plane of the finite element mesh. Unfortunately, such measurements are not captured in the present thesis because of great experimental difficulties that will be discussed in the present chapter.

The following paragraphs discuss each of the mentioned issues (reconstruction, experiments and model) in general, as far as a specific discussion is not yet captured in the concerning chapter. Where applicable, the text points into directions for future research. 


\section{Reconstruction}

In two chapters of this thesis, anatomically realistic finite element meshes are generated for use in simulations. A highly detailed reconstruction was created of the rat calf muscle and from these data, a three-dimensional mesh of the medial gastrocnemius muscle was obtained in chapter 5 . Despite, simulations in chapter 2 and 3 of this thesis are performed with a relatively simple, two-dimensional representation of the longitudinal crosssection of the medial gastrocnemius muscle. In fact, this two-dimensional mesh corresponds to the basic geometry of an unipennate muscle. Although it is based on the reconstruction, it could also have been generated without the efforts that were taken to obtain the detailed reconstruction. One reason for using this clementary mesh and not the realistic three-dimensional one is involved with the size of the mesh. Many elements are to be included in the mesh to capture the great detail of the three-dimensional reconstruction. This makes the mesh difficult to handle, while simulations take too much computational time. Another major shortcoming of the obtained reconstruction is the lack of muscle fiber direction information, which is one of the required input parameters for simulations. Including correct fiber directions is important for biomechanical studies to muscle functioning; it is of no use to incorporate highly detailed contours, when fiber directions are unrealistic.

The fact that finite element simulations did not use the great detail of the reconstruction questions the benefits of such reconstruction. Probably the most important advantage is the source of data that comes available, which can be used in any study that relies on geometrical data. With respect to finite element simulations, only the midsagittal section of the medial gastrocnemius muscle is presently used, but a mesh of for instance the soleus muscle or a cross-sectional representation of the gastrocnemius muscle can be derived from these data as well.

Moreover, a detailed mesh is essential when studying local deformation of muscle tissue or aponeurosis. Surface deformation during contractions, simulated with the provided three-dimensional mesh, can be compared to the strain fields during contraction, as determined in chapter 4. Probably, such simulations can be computed in the future.

The DTI method copes with the disadvantages of the previous method. Within a few hours, local three-dimensional muscle fiber directions are obtained. Together with high-resolution MRI data, these are used in chapter 6 to generate a finite element mesh with accurate fiber directions. There are some disadvantages of MRI as well. In contrast with the histological reconstruction method (chapter 5), borders between muscles are not very clear in high-resolution MR-images and it is difficult to tell one 
muscle from another. Further, the exact location of aponeuroses is hard to determine from MRI. This is mainly due to the large voxel size relative to aponeurosis thickness and to the size of the tissue layers that separate individual muscles. Reconstruction of approximate muscle contours from high-resolution MRI is only possible with à priori anatomical knowledge. This method proved to be successful for construction of an anterior tibialis mesh, but will probably fail in distinguishing between individual muscles of the anatomically more complicated triceps surae. The detailed reconstruction of chapter 5 will be valuable in that case.

Obviously, the benefits and disadvantages of the two described methods are dissimilar. Combination of the methods can provide all essential information to create a three-dimensional mesh including muscle fiber directions with sufficient detail. Using the great detail of histologic slides, a fairly accurate reconstruction of muscle contours in the plane of highresolution MR-images can be obtained. Fiber directions within this mesh can easily be added from DTI data, because coordinates of DTI measurements and high-resolution MRI correspond when these are acquired within one experimental setting.

\section{Experiments}

\section{Animal experiments}

Animal experiments in this thesis regard deformation and perfusion of skeletal muscle during contraction. With respect to deformation (chapter 4), three-dimensional displacements of markers attached to the muscle surface are calculated into longitudinal and transverse strain distribution at the aponeurosis and muscle surface. From these experiments was concluded that the effect of muscle length on contraction can only be studied when taking three dimensions into account. Regarding muscle perfusion (chapter 2), the effect of contraction on total afferent and efferent muscle blood flow is measured. Despite the large effect on arterial and venous flow, total volume change was only minimal during sustained isometric contractions. In combination with results of simulations, which compared qualitatively but not quantitatively to experiments, the conclusion was drawn that contraction most importantly affects the venous blood volume. The fact that veins play a prominent role in the interaction between contraction and perfusion is consistent with the vascular waterfall theory.

An extended discussion regarding the experiments is already performed in the concerning chapters. A general remark is that all measurements are performed in vivo and not in situ. This must be kept in mind when extrapolating the experimental data to in situ contractions. Environmental tissues, i.e. surrounding bones and muscles, may affect functioning of the 
concerned muscle. Therefore, muscle deformation and for instance the distribution of intramuscular pressure and related variables in these experiments may differ from muscle contractions in situ.

Future, comparable studies to muscle function, including deformation and perfusion, are recommended to leave this in vivo set-up and head for in situ measurements. At present, specially developed devices to measure rat lower leg muscle force, or actually ankle torque, in situ during isometric, concentric and eccentric contractions are available. These methods not only enable studying muscle functioning in situ, but the surgical intervention is less complicated, less radical and less time-consuming as well.

An interesting topic for future research would not only be to measure muscle function in situ, but also to investigate the effect of surrounding tissues on its behavior. This can be addressed by comparing the present experiments, performed in the draw-bench, to in situ experiments.

Regarding muscle deformation, two more recommendations for future research are the following. First, defurmation in the longitudinal muscle direction, presently chosen as representative for muscle fiber direction, should be described in the actual muscle fiber direction to study muscle fiber behavior during contractions. Further, principal strains can be compared to muscle fiber directions, to verify the hypothesis that the largest strains occur in the fiber direction. Second, in addition to muscle surface deformation measurements, intramuscular deformation should be assessed. Such measurements, which can be performed using tagging-MRI, will provide additional information on muscle deformation. Moreover, such data are easily comparable with numerical simulations with the available finite element meshes.

Spatial distributions of mechanical parameters

\section{General}

The numerical model of blood perfused contracting skeletal muscle has been used in the literature $(116,117)$ as well as in this thesis (chapters 2 and 3 ) to study muscle perfusion. Validation of this model was performed by comparing flow and blood pressure data of finite element simulations with a network simulation (115), and with outcome of a lumped parameter

Two separate devices are available at the Maastricht University, faculty of Movement Sciences. The first one can be used for measuring ankel torque during isometric contractions, while the other one is used to perform dynamic measurements (concentric or eccentric contractions) for rat lower leg muscles. The latter apparatus has been described in detail by Hesselink in chapter 2 of his thesis (39). Similar devices have recently become available for ankle torque measurements in mice. 
model (117). The present study provides a comparison of model simulations with experimental data of total muscle perfusion during contraction (chapter 2). A more comprehensive comparison between experiments and simulations involves the spatial distribution of any model parameter. Intramuscular pressure distribution is a suitable variable as it interacts between perfusion and contraction, but also, flow or pressure distribution within a certain blood compartment would do. These comparisons are not available in the present study, for great experimental difficulties of such measurements. Yet, because of the great benefits of such comparison for validation of the model, the next paragraphs discuss the possibilities with regard to distribution measurements of these parameters during contraction. First, a few general remarks regarding such experiments are made.

Measuring three-dimensional distributions of mechanical variables within skeletal muscle during contraction requires either penetration of the muscle at a number of locations or the use of non-invasive techniques like MRI, laser, or ultrasound. In both cases, muscle deformation during the measurement is the most prominent problem. With invasive techniques, there is a severe risk of muscle damage. With non-invasive techniques a major problem is that (part of) the muscle moves with respect to the measurement device. Special attention should be paid to the quick developments that take place in MRI. Techniques like tagging MRI provide ways to determine tissue deformation and thus to take muscle contraction into account. Another major general experimental difficulty is that forceful contractions cannot sustain very long. Therefore, measurements must be performed within a few seconds, which may become a problem with a number of techniques.

\section{Intramuscular pressure}

Intramuscular pressures in contracting muscles reported in the literature, differ. Disagreements can largely be explained by differences in muscle geometry (109) or measurement location, as intramuscular pressure varies with depth $(53,74,86,91)$. However, differences also result from artifacts, that are unquestionably introduced in all available methods, like fiber rupture or fiber distortion at the location where the measurement device is introduced in the muscle (5).

The variety of measurement techniques is discussed in detail by Sejersted and Hargens (90). Common problems with techniques for monitoring intramuscular pressure during contractions are that they respond slowly to pressure changes (wick catheters), or cannot cope with the muscle deformation (micropipettes). Only using transducer-tipped catheters, rapid changes can be measured. 
For comparison of intramuscular pressure distribution between finite element simulations and experiments, a series of measurements must be performed at different locations throughout the contracting muscle. For experimental difficulties, the variability between measurements and the mentioned artifacts, this can hardly be done reliably. Yet, for the cintral role of tissue pressure in the finite element model, aiming at such measurements is worth the effort in future studies.

\section{Blood flow distribution}

Ultrasound and laser techniques are used in clinical and experimental settings to determine local blood flows. Further, MRI techniques have become available that can be used to measure the distribution of blood flow throughout a structure. All these methods have one genteral disadvantign. with respect to contraction: they do not account for tissue deformation. Therefore, measuring flow differences between contraction and relaxation at a certain location in the tissue is impossible.

A comprehensive and generally used method to measure blood flow in hierarchical structures like the lungs and the vascular tree is by means of microspheres $(2,27,38,62,82,121)$. Briefly, this method works as follows. Spheres injected into the arterial system are transported by the blood and finally trapped in vessels of the approximate sphere-diameter. Assuming full mixture and appropriate spheres size, local blood flow during the experiment is proportional to the number of deposited microspheres (11).

The microspheres method has a number of prominent advantages. First, measurements can be performed in situ, without the risk of damaging the muscle by preparation, and it is independent of deformation. Second, differently labeled radioactive, dyed or fluorescent spheresi can be used in the same experiment to distinguish between experimental conditions within the same muscle. In this way, the muscle with its specific geometry, size and vascular tree becomes its own reference when comparing experimental conditions.

Many studies have dealt with the accuracy and reliability of this method (6, $24,34,40,98)$. Principally, the method is suitable for its aim: to determine the capillary flow distribution at rest and during contraction, and to compare these data to simulations. However, for a number of statistical and practical considerations this method does not work out in the present study.

Regarding statistics of the microspheres method, a few facts follow. First, approximately 400 microspheres are needed per tissue sample for reliable blood flow estimation (24). Second, maximally $2 \%$ of the vessels may be occluded, else redistribution of blood flow would occur and measurements become inaccurate. Third, approximately 1100 capillaries per $\mathrm{mm}^{3}$ exist in 
skeletal muscle (21), which equals $\sim 3.3^{\star} 10^{6}$ capillaries per 3 grams weighting rat calf muscle. Thus, $6.6^{*} 10^{4}$ capillaries may be occluded maximally during one experiment and $6.6^{\star} 10^{4} / 400=165$ tissue samples can maximally be obtained from one muscle provided that spheres are homogeneously distributed. Usually, the minimum number of spheres per sample is doubled to make sure that an adequate number is deposited in low flow pieces (24). At first sight, 165/2=82 samples per muscle seems sufficient to determine flow distribution. However, for the inhomogene flow distribution during muscle contraction, parts of the muscle contain far less spheres than other parts and it is not a priori known which parts receive less flow. Therefore, tissue samples of identical sizes should at least be several times the size. Five times the sample size leaves at most $82 / 5=16$ samples. If separate conditions are to be distinguished, which are at least perfusion at rest and during contraction for proper comparison, only half the number of spheres can be used per condition, leaving only 8 samples in the case the vascular tree is filled with exactly the maximum amount of spheres. At least three, but preferably more than five samples per muscle are needed for proper presentation of flow distribution. Thus, although it is principally possible to obtain the desired information, the injected number of spheres and muscle perfusion must be in close harmony to obtain valid results. The dependence of muscle perfusion on contraction force makes it even more difficult to determine the appropriate number of spheres to inject during contraction. The easiest solution to these problems is to use larger muscles, i.e. another animal model.

The second reason why the microspheres method is difficult to use in the present study concerns timing. Forceful contractions cannot last for more than a couple of seconds and spheres will have to reach the capillaries within this period to represent blood flow distribution during contraction. However, for the time the injection takes and for the distance between the place of injection and the organ, necessary for proper mixing of the spheres, the first microspheres reach the muscle seconds before the last ones do. After entering the muscle, it takes a few more seconds before spheres are deposited. Since maximum contraction forces cannot sustain that long, weaker contractions must be used, which are expected to result in less obvious differences between contraction and relaxation. This means that more spheres and/or more experiments are needed to draw reliable conclusions.

Thus, although the microspheres method principally provides a way to obtain the required data, the experimental set-up is critical and numerous pilot experiments are needed. Each time, microspheres distribution in the muscle must be analyzed to redirect experimental conditions. Unfortunately, localization of microspheres after the experiment is timeconsuming. A generally used method to determine fluorescent 
microspheres deposition is to dissolve small pieces of tissue, isolate the pallet of spheres, dissolve the spheres in a known volume and measure fluorescent intensity of the solution. This procedure takes a couple of days. The two main disadvantages are that spatial information is restricted to the number of tissue pieces and that tissue sample size needs to be determined in advance. Localizing the three-dimensional coordinates of all spheres copes with these disadvantages. To obtain these coordinates manually is a laborious task. Currently, machines have become available that automate the procedure of three-dimensionally localizing individual fluorescent microspheres, by a combination of a cryotome and video with fluorescent filters.

A recommendation for further studying blood flow distribution is to use the microsphere method in larger muscles and acquire data with an automated cryotome. An estimate of the number of spheres to inject at rest and during contraction, can be derived from the expected total flow during contraction (chapter 2) and the heterogeneity of flow distribution (chapter 3 ). Force during the experiments can be controlled by means of an apparatus, similar to the aforementioned one to measure rat ankle torque during contraction.

Model

\section{Validity}

The presently used finite element model of perfused contracting skeletal muscle has specially been developed to calculate the mechanical effect of contraction on the spatial distribution of physiological perfusion parameters throughout a skeletal muscle. The model constitution has extensively been described in the literature $(112,114,115)$. Simulations were compared to animal experiments by Vankan et al $(116,117)$ and in chapter 2, where simulations were also used to interpret experiments. In chapter 3 , the interaction between contraction, intramuscular pressure and regional tissue perfusion was calculated, which is impossible to access experimentally. Despite, the only actual model validation study addresses the bare flow description and compartmentalization of the fluid constituent, via comparison of a finite element simulation with a network simulation of an identical vascular tree (115). This validation study does not comprise an interaction between tissue pressure and perfusion, which is the most essential interaction in the model with respect to its physiological importance.

The large number of variables in the finite element model makes it hard to validate the model properly. It includes tissue pressure and six blood pressures, surrounding the five blood compartments arteries, arterioles, 
capillaries, venules and veins. Each blood compartment is described by its porosity, which equals its local blood volume relative to the local tissue volume, permeability in spatial and hierarchical direction, which describes the ease of fluid flow in these directions, and the relationship between transmural pressure and porosity, which relates to vessel wall properties. These variables need to be defined per element. One may see this number of variables as an unlimited possibility to fit simulations to experiments, therewith devaluating the model. Skepticism regarding extended use of the model to calculate phenomena that cannot be measured experimentally is the result.

However, despite the great difficulties to accurately validate the model with experiments, the model is found trustworthy for a number of reasons. First, input data relate to experimentally determinable parameters with a physiological meaning, while many of these parameters, like tissue porosity and permeability, are obtained from the literature. Other variables can be estimated reliably; only few settings remain questionable. Among these are the relationships between porosity and transmural pressure during extravascular pressure rise. Regarding model output, suitable reference parameters can be found in for instance hierarchical blood pressure distribution. The use of completely wrong parameter settings affects thesc standard references. Further, although not an actual validation, comparison of simulation results with experimental data, as done by Vankan et al (116, $117)$ and in chapter 2 of this thesis, does provide insight into the model integrity. Some characteristics of total arterial and venous flow patterns are essentially present in simulations and experiments. The venous flow pattern during the first second of relaxation for instance, was at first thought to be an artifact of the flow measurement, but was gently accepted when noticed in simulations.

Yet, close comparison between experiments and simulations does not mean that all input parameters are correct, or that calculated local flow or compartment porosity is physiologically realistic. Therefore, too many variables are to be set in the model. Obviously, quantitative differences between simulations and experiments exist between total flow signals (chapter 2). In turn, this does not mean that the model is not to be used in future studies. A major issue in using these kinds of models is to find out which part of simulations to interpret physiologically. Blood volumes per compartment, hierarchical pressure distribution (Figure 2.8) and total perfusion at rest correspond to literature values (chapter 2). During contraction, flow patterns of simulations qualitatively agree with experiments, but the calculated quantitative effect of contraction on total flow and blood volume change exceeds experimental data. Therefore, merely qualitative conclusions have been drawn from simulations where these involve contractions (chapter 2 and 3 ). 
Quantitative differences during contraction can be redirected to excessive outflow of blood in simulations, which merely comes to the account of venous blood volume (chapter 2). The latter chapter points into various directions of model adjustment, which may decrease outflow during contraction and result in a closer comparison between experiments and simulations. Two of the main suggestions regard compartmentalization of the vascular tree and the assumptions of continuum and homogeneity for large vessels. Actually, the need for reconsidering these assumptions becomes more important when the number of compartments is increased. The smaller the compartments, the more likely the distribution of permeability and porosity within the compartments of large vessels is neither homogeneous nor continuous.

A final remark on the aforementioned suggestions of chapter 2 regard the spatial interactions between perfusion and contraction that are described in chapter 3 . When predominant pathways for spatial flow are included in the model (chapter 2), spatial interactions (chapter 3) may become more important to muscle perfusion. It is recommended to pay attention to the compartmentalization of the vascular tree and to the assumptions of continuum and homogeneity in further studies.

\section{Geometry}

The input variable that has given the most attention in this thesis is musile: geometry. Discussion regarding the need and usability of detailed anatomical reconstruction is already provided for in previous paragraphs. One remark regarding simulations is to be made here. Chapters 2 and 3 use a finite element mesh of the midsagittal section through the medial gastrocnemius muscle, whereas in chapter 6, a mesh of the anterior tibialis muscle is used. Actually, the anterior tibialis muscle seems more suitable for finite element studies to muscle function, because it is anatomically located in its own compartment together with the relatively small long extensor digitorum muscle. Therefore, the TA functions much more like an individual muscle than the medial gastrocnemius muscle does, which is basically one-third part of the triceps surae muscle.

However, most simulations in this thesis address the medial gastrocnemius muscle, because they are intended to compare to experimental measurements. Perfusion measurements are performed on the triceps surae muscle, because blood vessels can easier be isolated and have sufficient length, whereas feeding vessels of the anterior tibialis muscle are shorter and difficult to approach. For surface deformation measurements, it is important to free the muscle surface from fascia. This can be done for the surface of the medial gastrocnemius muscle, but the well-developed fascia of the anterior tibialis muscle can hardly be removed sufficiently. On top, 
because the fascia of the anterior tibialis muscle is much better developed, removal is expected to affect muscle functioning more importantly.

Because simulations of the TA muscle reflect muscle functioning better than simulations of the GCM do, the TA muscle should be paid more attention in future studies. Preferably, such measurements should be performed in close harmony with in situ deformation or perfusion measurements. In this respect, MRI techniques need further attention.

\section{Model simulations vs. physiological muscle functioning}

The discussion regarding experimental and modeling (dis)agreements leads to the question to what extend the model does commit to the functioning of a skeletal muscle. The model is built to calculate contraction and perfusion, and merely the local interaction between these two via the effect of intramuscular pressure building on blood pressure, intermediated by vessel wall properties.

The importance of the use of physiologically interpretable parameters and relationships in the description of perfusion, must be stressed. Vessel wall properties and muscle geometry are input parameters, whereas deformation, blood pressure and blood volume distributions result. The advantage of this approach includes the possibility to directly compare experiments and simulations, with respect to both in- and output parameters. Further, this particular use of parameters probably benefits acceptance of the model by experimental co-workers and also benefits discussion regarding the data between modelers and physiologists.

In contrast with the description of perfusion, the contraction model has not been given much attention. It is merely a prescription of an extra stress component in a particular (fiber) direction, based on the description Bovendeerd used for cardiac muscle contraction (20, 114). Advanced mathematical descriptions of contraction, based on for instance the sliding filament theory, should be incorporated in future studies to muscle functioning (33). This has not been done, because the present study does not focus at contractions, but merely at the interaction between tissue pressure and perfusion.

Furthermore, the finite element description of contraction as used by Gielen (33) provides possibilities in studying the interaction between local muscle fiber activation and the availability of metabolites like calcium and ATP. If this description were added to the present model of local tissue perfusion, an actual interaction between local blood supply and energy consumption is within reach. This principally provide ways to study for instance muscle fatigue and bring the present model closer to muscle physiology. 


\section{CONCLUSIONS}

Total arterial and venous muscle flow decrease with increasing tetanic contraction force; mean arterial inflow decreases to $10 \%$ of the initial flow level during forceful contraction. Vascular volume change during contraction is only small, but blood volume still decreases after $800-\mathrm{ms}$ contraction. Simulations with the finite element model of perfused contracting skeletal muscle qualitatively compare to perfusion experiments, but differ quantitatively. From simulations is derived that most initial volume change is located in the veins, whereas the volume of other compartments steadily decreases when contraction sustains.

The vascular waterfall behaves differently during muscle contraction as compared to pressure box experiments. Spatial interactions butween contraction, intramuscular pressure and local tissue perfusion are responsible. It is concluded that geometrical effects must h. winsidered before extrapolating the principles of the vascular waterfall theory, derived from pressure box experiments, to contracting skeletal muscle.

Longitudinal and transverse strains during isometric contraction decrease with increasing muscle length. Mean transverse strain at the aponeurosis equals twice the mean transverse strain at the superficial muscle fibers. Further, aponeurosis surface area change depends on muscle length, whereas muscle surface area change is $-11 \%$, independent of muscle length. Assuming isovolumic muscle deformation, strain perpendicular to the muscle surface equals $11 \%$ at either muscle length. Changes in longitudinal strain are accounted for by the transverse strain. Models that do not account for these three-dimensional deformation cannot be used to calculate these effects of muscle length changes on mechanical muscle: functioning.

To enable the use of three-dimensional meshes in finite element models, a detailed reconstruction of rat triceps surae muscle is created, based on histologic slides. From these data a three-dimensional finite element mesh of the medial gastrocnemius muscle is derived, which can be used in finite element simulations. This model lacks accurate fiber directions.

Local anisotropy direction as determined by diffusion weighted MRI does represent the local muscle fiber direction within 5 degrees accuracy in voxels larger than $0.9 \mathrm{~mm}^{3}$. In combination with high-resolution $\mathrm{MR}$ images, this technique is suitable to generate a two-dimensional geometrical reconstruction of a skeletal muscle that includes accurate fiber directions for use in biomechanical studies to muscle functioning. A twodimensional mesh of the anterior tibialis muscle is obtained with this method, and is used in a simulation as an illustration. 


\section{LITERATURE}

1. Flowmeters for Medicine \& Research. Transonic Systems Inc., A.B. Medical b.v., 1995.

2. Armstrong, R. B., and M. H. Laughlin. Rat muscle blood flows during high-speed locomotion. J Appl Physiol59: 1322-1328, 1985.

3. Armstrong, R. B., and R. O. Phelps. Muscle fiber type composition of the rat hindlimb. Am JAnat 171: 259-272, 1984.

4. Arts, T. A mathematical model of the dynamics of the left ventricle and the coronary circulation. University of Limburg, Maastricht, The Netherlands. (Thesis) 1978.

5. Arts, T., and R. S. Reneman. Interaction between intramyocardial pressure (IMP) and myocardial circulation. J Biomech Eng 107: 51-56, 1985.

6. Austin, R. E., W. W. Hauck, G. S. Alsea, A. E. Flynn, D. L. Coggins, and J.I. E. Hoffman. Quantitating error in blood flow measurements with radioactive microspheres. Am J Physiol 257: H280-H288, 1989.

7. Badeer, H. S., and J. W. Hicks. Hemodynamics of vascular 'waterfall': is the analogy justified? Respir Physiol 87: 205-217, 1992.

8. Barcroft, H., and J. L. E. Millen. The blood flow through muscle during sustained contraction. / Physiol 97: 17-31, 1939.

9. Basser, P. J., J. Mattiello, and D. LeBihan. Estimation of the effective self-diffusion tensor from the NMR spin echo. J Magn Res 103: 247-254, 1994.

10. Basser, P. J., J. Mattiello, and D. LeBihan. MR diffusion tensor spectroscopy and imaging. Biophys J66: 259-267, 1994.

11. Bassingthwaithe, J. B., M. A. Malone, T. C. Moffett, R. B. King, I. S. Chan, J. M. Link, and K. A. Krohn. Molecular and particulate depositions for regional myocardial flows in sheep. Circ Res 66: 1328-1344, 1990.

12. Bellamy, R. F. Calculation of coronary vascular resistance. Cardiovasc Res 14: 261-269, 1980.

13. Best, T. M., J. H. McElhaney, W. E. Garrett, and B. S. Myers. Axial Strain Measurements in Skeletal Muscle at Various Strain Rates. Trans ASME117: 262-265, 1995.

14. Blix, M. Die länge und spannung des muskels. Skand Arch Physiol 111: 295-318, 1883.

15. Blix, M. Die lănge und spannung des muskels: dritte abhandlung. Skand Arch Physiol V: $150-172,1884$.

16. Blix, M. Die länge und spannung des muskels: vierte abhandlung.Skand Arch Physiol V: $180-206,1884$.

17. Blix, M. Die länge und spannung des muskels: zweite abhandlung.Skand Arch Physiol IV: $400-409,1883$.

18. Bovendeerd, P. H. T. Arts, J. M. Huyghe, D. H. van Campen, and R. S. Reneman.

Dependence of local left ventricular wall mechanics on myocardial fiber orientation: a model study, JBiomech 25: 1129-1140, 1992.

19. Bovendeerd, P. H., J. M. Huyghe, T. Arts, D. H. van Campen, and R. S. Reneman. Influence of endocardial-epicardial crossover of muscle tibers on left ventricular wall mechanics. / Biomech 27: 941-951, 1994.

20. Bovendeerd, P. H. M. The mechanics of the normal and ischemic left ventricle during the cardiac cycle. Department of Biophysics, University of Limburg Maastricht, The Netherlands. (Thesis) 1990. 
21. Braakman, R., P. Sipkema, and N. Westerhof. A dynamic nonlinear lumped parameter model for skeletal muscle circulation. Ann Biomed Engng 17: 593-616, 1989.

22. Braakman, R., P. Sipkema, and N. Westerhof. Two zero-flow pressure intercepts exist in autoregulating isolated skeletal muscle. Am J Physio/258: H1806-H1814, 1990.

23. Bruinsma, P., T. Arts, J. Dankelman, and J. A. Spaan. Model of the coronary circulation based on pressure dependence of coronary resistance and compliance. Basic Res Cardiol83: 510-524, 1988.

24. Buckberg, G. D., J. C. Luck, D. B. Payne, J. I. E. Hoffman, J. P. Archie, and D. E. Fixler. Some sources of error in measuring regional blood flow with radioactive microspheres. J Appl Physiol 31: 598-604, 1971.

25. Cleveland, G. G., D. C. Chang, C. F. Hazlewood, and H. E. Rorschach. Nuclear magnetic measurement of skeletal muscle. Biophys J16: 1043-1053, 1976.

26. Coremans, J., R. Luypaert, F. Verhelle, T. Stadnik, and M. Osteaux. A method for myelin fiber orientation mapping using diffusion-weighted MR images Magn Reson Imaging 12: 443-454, 1994.

27. Delp, M. D., and R. B. Armstrong. Blood flow in normal and denervated muscle during exercise in conscious rats. Am J Physiol 255: H1509-H1515, 1988.

28. Downey, J. M., and E. S. Kirk. Inhibition of coronary blood flow by a vascular waterfall mechanism. Circ Res 36: 753-760, 1975.

29. Engstrom, C. M., G. E. Loeb, J. G. Reid, W. J. Forrest, and L. Avruch. Morphometry of the human thigh muscles. A comparison between anatomical sections and computer tomographic and magnetic resonance images. J Anat 176: 139-156, 1991.

30. Folkow, B., P. Gaskell, and B. A. Waaler. Blood flow through limb muscles during heavy rhythmic exercise. Acta Physiol Scand 80: 61-72, 1970.

31. Gareis, H., M. Solomonow, R. Baratta, R. Best, and R. D'Ambrosia. The isometric length-force models of nine different skeletal muscles./ Biomech 25: 903-916, 1992.

32. Garrido, L., V. J. Wedeen, K. K. Kwong, U. M. Spencer, and H. L. Kantor. Anisotropy of water diffusion in the myocardium of the rat. Circ Res 74: 789-793, 1994.

33. Gielen, A. W. J. A continuum approach to the mechancis of contracting skeletal muscle. Department of Mechanical Engineering, Eindhoven University of Technology, Eindhoven, The Netherlands. (Thesis) 1998.

34. Glenny, R. W., S. Bernard, and M. Brinkley. Validation of fluorescent-labled microspheres for measurement of regional organ perfusion./ Appl Physiol: 2585-2597, 1993.

35. Gordon, A. M., A. F. Huxley, and F. J. Julian. The length-tension diagram of single vertebrate striated muscle fibers. J Physiol171: 28P, 1964.

36. Gray, S. E., E. Carlsson, and N. Staub. Site of increased vascular resistance during isometric muscle contraction. Am J Physiol213: 683-689, 1967.

37. Griffiths, R. I. Shortening of Muscle Fibres during Stretch of the Active Cat Medial Gastrocnemius Muscle: the Role of Tendon Compliance.J Physio/436: 219-236, 1991.

38. Hargreaves, D., S. Egginton, and O. Hudlicka. Changes in capillary perfusion induced by different patterns of activity in rat skeletal muscle. Microvasc Res 40: 14-28, 1990.

39. Hesselink, M. K. C. Structural, functionai and metabolic aspects of shortening and lengthening muscle contractions. Deptartment of Movement Sciences, Maastricht University, Maastricht, The Netherlands. (Thesis) 1998.

40. Heyman, M. A., B. D. Payne, J. I. E. Hoffman, and A. M. Rudolph. Blood flow measurements with radionuclide-labeled particles. Prog Cardiovasc Dis 20: 55-79, 1977. 
41. Hill, A. V. The heat of shortening and the dynamic constants of muscle. Proc Roy Soc Lond 126: 136-195, 1938.

42. Hill, A. V. The pressure developed in muscle during contraction.JPhysiol 107: 518-526, 1948.

43. Hirche, H. J., W. K. Raff, and D. Grun. The resistance to blood flow in the gastrocnemius of the dog during sustained and rhythmical isometric and isotonic contractions. Pflugers Arch 314: 97-112, 1970.

44. Holewijn, M., P. Plantinga, R. Woittiez, and P. Huijing. The number ofsarcomeres and architecture of the $\mathrm{m}$. gastrocnemius of the rat. Acta Morphol Neerl-Scand 22: 257-263, 1984.

45. Hsu, E. W., A. L. Muzikant, S. A. Matulevicius, R. C. Penland, and C. S. Henriquez. Magnetic resonance myocardial fiber-orientation mapping with direct histological correlation. Am J Physio/ 274: H1627-H1634, 1998.

46. Huijing, P. A., and G. J. Ettema. Lenght-force characterisctics of aponeurosis in passive muscle and during isometric and slow dynamic contractions of rat gastrocnemius muscle. Acta Morphol Neerl-Scand 26: 51-62, 1988/89.

47. Huijing, P. A., and R. D. Woittiez. Length range, morphology and mechanical behaviour of rat gastrocnemius muscle during isometric contraction at the lever of the muscle and mu. Neth J Zool35: 505-516, 1985.

48. Humphrey, J. D. Mechanics of the arterial wall: review and directions. Crit Rev Biomed Eng 23: 1-162, 1995.

49. Huxley, A. F. Muscle structure and theories of contraction. Prog Biophys Biophys Chem 7: 255-318, 1957.

50. Huyghe, J. M. Non-linear finite element models of the beating !eft ventricle and the intramyocardial coronary circulation. Eindhoven University of Technology, Eindhoven, The Netherlands. (Thesis) 1986.

51. Huyghe, J. M., T. Arts, D. H. van Campen, and R. S. Reneman. Porous medium finite element model of the beating left ventricle. Am J Physiol 262: H1256-H1267, 1992.

52. Huyghe, J. M., D. H. van Campen, T. Arts, and R. M. Heethaar. A two-phase finite element model of the diastolic left ventricle.J Biomech 24: 527-538, 1991.

53. Jensen, B. R., K. Jorgensen, P. A. Huijing, and G. Sjogaard. Soft tissue architecture and intramuscular pressure in the shoulder region. Eur /Morphol33: 205-220, 1995.

54. Julian, F. J., and D. L. Morgan. The effect on tension of non-unitorm distribution of length changes applied to frog muscle fibres. P Physiol Lond 293: 379-392, 1979.

55. Katz, B. The relation between force and speed in muscular contraction.J Physio/96: 45 64,1939 .

56. Kirkebo, A., and A. Wisnes. Regional tissue fluid pressure in rat calf musde during sustained contraction or stretch. Acta' Physiol Scand 114: 551-556, 1982.

57. Krarns, R., P. Sipkema, and N. Westerhof. Varying elastance concept may explain coronary systolic flow impediment. Am J Physio/257: H1471-H1479, 1989.

58. Krams, R., A. C. T. A. Van Haelst, P. Sipkema, and N. Westerhof. Can coronary systolicdiastolic flow differences be predicted by left ventricular pressure or time-varying intramyocardial elastance? Bas Res Cardiol 84: 149-159, 1989.

59. Lieber, R. L., M. E. Leonard, C. G. Brown, and C. L. Trestik. Frog Semitendinosis Tendon Load-strain and Stress-strain Properties during Passive Loading. Am JPhysio/261: C86-C92, 1991.

60. Livingston, J. Z., J. R. Resar, and F. C. Yin. Effect of tetanic myocardial contraction on coronary pressure-flow relationships. Am J Physiol 265: H1215-H1226, 1993. 
61. Magder, S. Venous mechanics of contracting gastrocnemius muscle and the muscle pump theory. J Appl Physiol79: 1930-1935, 1995.

62. Marconi, C., N. Heisler, M. Meyer, H. Weitz, D. R. Pendergast, P. Cerretelli, and J. Piiper. Blood flow distribution and its temporal variability in stimulated dog gastrocnemius muscle. Resp Physiol 74: 1-14, 1988.

63. Marshall, $\mathrm{J}$., and $\mathrm{H}$. Tandon. Direct observations of muscle arterioles and venules following contraction of skeletal muscle fibres in the rat. $J$ Physiol $350: 447-459,1984$.

64. McLean, M., and J. Prothero. Determination of relative fiber arientation in heart muscle: methodological problems. Anat Rec 232: 459-465, 1992.

65. Meijer, K., H. J. Grootenboer, H. F. J. M. Koopman, and P. A. Huijing. Length-force curves during and after concentric contractions differ from the fully isometric length-force curve in rat muscle. J Appl Biomech 13: 164-181, 1997.

66. Mellander, $S_{\text {.r }}$ and U. Albert. Effects of increased and decreased tissue pressure on haemodynamic and capillary events in cat skeletal muscle.J Physiol Lond 481: 163-175, 1994.

67. Moseley, M. E., Y. Cohen, J. Kucharczyk, J. Mintorovitch, H. S. Asgari, M. F. Wendland, 1. Tsuruda, and D. Norman. Diffusion-weighted MR imaging of anisotropic water diffusion in cat central nervous system. Radiology 176: 439-445, 1990.

68. Muijtiens, A. M. M., J. M. A. Roos, T. Arts, and A. Hasman. 3-D Reconstruction of marker positions from stereo images using ML estimation. Froc Conf on Medical and Biological Engineering, Jerusalem, 17-21 Sept, 1995.

69. Muijtiens, A. M. M. J. M. A. Roos, T. Arts, A. Hasman, and R. S. Reneman. Extrapolation of incomplete marker tracks by lower rank approximation.Int J Biomed Comput 33: 219-239, 1993.

70. Muijtiens, A. M. M., J. M. A. Roos, T. Arts, A. Hasman, and R. S. Reneman. Simultaneous estimation of stereo correspondence and camera geometry from marker tracks. Proc IEEE Conf Comput Cardiol, 10-13 sept, Wenen. IEEE Computer Society Press, Washington, 1995, p. 577-580.

71. Muijtiens, A. M. M., J. M. A. Roos, T. Arts, A. Hasman, and R. S. Reneman. Tracking markers with missing data by lower rank approximation./ Biomech 30: 95-98, 1997.

72. Naamani, R., S. N. Hussain, and S. Magder. The mechanical effects of contractions on blood flow to the muscle. Eur J Appl Physio/71: 102-112, 1995.

73. Nakada, T., and H. Matsuzawa. Three-dimensional anisotropy contrast magnetic resonance imaging of the rat nervous system: MR axonography. Neurosci Res 22: 389-398, 1995.

74. Nakhostine, M., J. R. Styf, S. van Leuven, A. R. Hargens, and D. H. Gershumi. Intramuscular pressure varies with depth. The tibialis anterior muscle studied in 12 volunteers. Acta Orthop Scand 64: 377-381, 1993.

75. Otten, E. Concepts and models of functional architecture in skieletal muscle. Exerc Sport Sci Rev 16: 89-137, 1988.

76. Patel, T. J., and R. L. Leiber. Force transmission in skeletal muscle; from actomyosin to external tendons. Excerc Sport Sci Rev 25: 321-363, 1997.

77. Pendergast, D. R., J. A. Krasney, A. Ellis, B. McDonald, C. Marconi, and P. Cerretelli. Cardiac output and muscle blood flow in exercising dogs. Resp Physiol 61: 317-326, 1985.

78. Permutt, S., and R. L. Riley. Hemodynamics of collapsible vessels withtone: the vascular waterfail. J.Appl Phys 18: 924-932, 1963.

79. Peters, G. W. M. Tools for measurement of stress and strain ficlds in soft tissue. Department of Anatomy and Embryology, University of Limburg, Maastricht, The Netherlands. (Thesis) 1987. 
80. Petrofsky, J. S., and D. M. Hendershot. The interrelationship between blood pressure, intramuscular pressure, and isometric endurance in fast and slow twitch skeletal muscle in cat. Eur J Alppl Physiol53: 106-111, 1984.

81. Pierpaoli, C., and P. J. Basser. Toward a quantitative assessment of diffusion anisotropy. Magn Reson Med 36: 893-906, 1996.

82. Piiper, J., C. Marconi, N. Heisler, M. Meyer, H. Weitz, D. R. Pendergast, and P. Cerretelli. Spatial and temporal variability of blood flow in stimulated doggastrocnemius muscle. Adv Exp Med Bio/ 248: 719-728, 1989.

83. Pries, A. R., T. W. Secomb, P. Gaehtgens, and J. F. Gross. Blood flow in microvascular networks. Experiments and simulation. Circ Res 67: 826-834, 1990.

84. Reese, T. G., R. M. Weisskoff, R. N. Smith, B. R. Rosen, R. E. Dinsmore, and V. J. Wedeen. Imaging myocardial fiber architecture in vivo with magnetic resonance.Magnt Reson Med 34: 786-791, 1995.

85. Reneman, R. S., D. W. Slaaf, L. Lindbom, G. J. Tangelder, and K. E. Arfors. Muscle blood flow disturbances produced by simultaneously elevated venous and total muscle tissue pressure. Microvasc Res 20: 307-318, 1980.

86. Sadamoto, T., F. Bonde Petersen, and Y. Suzuki. Skeletal muscle tension, flow, pressure, and EMG during sustained isometric contractions in humans. Eur J A Ppl Physiol51: 395-408, 1983.

87. Savelberg, H. H., and H. C. Schamhardt. The influence of inhomogeneity in architecture on the modelled force-length relationship of muscles. J Biomech 28(2): 187-197, 1995.

88. Scott, S. H., C. M. Engstrom, and G. E. Loeb. Morphometry of human thigh muscles. Determination of fascicle architecture by magnetic resonance imaging. I Anat 182: 249-257, 1993.

89. Scott, S. H., and G. E. Loeb. Mechanical properties of aponeurosis and tendon of the cat soleus muscle during whole-muscle isometric contractions.J Morpho/224: 73-86, 1995.

90. Sejersted, O. M., and A. R. Hargens. Intramuscular pressures for monitoring different tasks and muscle conditions. Adv Exp Med Biol384: 339-350, 1995.

91. Sejersted, O. M., A. R. Hargens, K. R. Kardel, P. Blom, O. Jensen, and L. Hermansen. Intramuscular fluid pressure during isometric contraction of human skeletal muscle.J Appl Physiol56: 287-295, 1984.

92. Sheriff, D. D., L. B. Rowell, and A. M. Scher. Is rapid rise in vascular conductance at onset of dynarnic exercise due to muscle pump? Am J Physio/265: H1227-H1234, 1993.

93. Shrier, I., and S. Magder. Maximal vasodilation does not eliminate the vascular waterfall in the canine hindlimb./ Appl Physiol79: 1531-1539. 1995.

94. Shrier, I., and S. Magder. Pressure-flow relationships in in vitro model of compartment syndrome. ' Appl Physiol 79: 214-221, 1995.

95. Slaaf, D. W., R. S. Reneman, and C. A. Wiederhielm. Cessation and onset of muscle capillary fiow at simultaneously reduced perfusion and transmural pressure. Int/Microcirc Clin Exp 6: 215-224, 1987.

96. Spaan, J. A. E., N. P. W. Breuls, and J. D. Laird. Diastolic-systolic coronary flow differences are caused by intramyocardial pump action in the anaesthetized dog. Circ Res 24: 339-347, 1981.

97. Spoor, C. W., J. L. van Leeuwen, W. J. van der Meulen, and A. Huson. Active forcelength relationship of human lower-leg muscles estimated from morphological data: a comparison of geometric muscle models. Eur J Morphol 29: 137-160, 1991. 
98. Stanek, K., T. Coleman, T. Smith and W. Murphy. Two hemodynanic problems commonly associated with the microsphere techique for measuring regional blood flow in rats. /Pharmaco/ Meth 13: 117-124, 1985.

99. Stejskal, E. O., and ]. E. Tanner. Spin diffusion measurements: spin echos in the presence of a time-dependent field gradient. J Chem Phys 42: 288-292, 1965.

100. Street, S. F. Lateral force transmission of tension in frog myofibers: a myofibrillar network and transverse cytoskeletal connections are possible transmitters. / Cell Physio/ 114: 346-364, 1983.

101. Trestik, C. L. and R. L. Lieber. Relationship between Achilles tendon mechanical properties and gastrocnemius muscle function. B Biomech Eng 115: 225-230, 1993.

102. Van Bavel, H., M. R. Drost, I. D. Wielders, J. M. Huyghe, A. Huson, and I. D. Janssen. Strain distribution on rat medial gastrocnemius (MG) during passive stretch.J Biomech 29: $1069-1074,1996$.

103. Van Bavel, H., C. A. J. Holzer, J. Van Leeuwen A. M. M. Muigtjens, P. J. B. Willems, and M. R. Drost. Quantification of local surface strains of cat gastrocnemius medialis during contraction. Neth J Zool $48,1998$.

104. Van Donkelaar, C.C., M. R. Drost, V. M. H., T. C.F. J. D. Janssen, and A. Huson. Threedimensional reconstruction of the rat triceps surae muscle and finite element mesh generation of the gastrocnemius medialis muscle. Eur fMorpo/34: 31-37, 1996.

105. Van Donkelaar, C. C., L. J. G. Kretzers, P. H. M. Bovendeerd, L. M. A. Lataster, K. Nicolay, I. D. Janssen and M. R. Drost. Diffusion tensor imaging in biomechanical studies of skeletal muwscle function. J Anat 194: 79-88, 1999.

106. Van Doorn, A., P. H. Bovendeerd, K. Nicolay, M. R. Drost, and J. D. Janssen. Determination of muscle fibre orientation using Diffusion-Weighted MRI.Eur JMorphol 34: 5-10, 1996.

107. Van Leeuwen, J. L., and C. W. Spoor. Modelling mechanically stable muscle architectures. Philos Trans R Soc Lond B Biol Sci336: 275-292, 1992.

108. Van Leeuwen, J. L., and C.W. Spoor. Modelling the pressure and force equilibrium in unipennate muscles with in-line tendons. Philos Trans R Soc Lond B Biol Sci 342: 321-333, 1993.

109. Van Leeuwen, J. L., and C. W. Spoor. A two dimensional model for the prediction of muscle shape and intramuscular pressure. Eur J Morphol34: 25-30, 1996.

110. Van Mameren, H., A. Lataster, H. Rensema, and J. Drukker. The use of modern imaging techniques (CT-scanning and NMR) in the study of the locomotor apparatus. Acta Morphol Neerl-Scand 22: 247-258, 1985.

111. Vankan, W. J. A Finite Element Model of Blood Perfused Contracting Skeletal Muscle. Dept. of Mechanical Engineering, Eindhoven University of Technology. The Netherlands. (Thesis) 1996.

112. Vankan, W. J., J. M. Huyghe, M. R. Drost, J. D. Janssen, and A. Huson. A finite element mixture model for hierarchical porous media. Int J Num Meth Eng 40: 193-210, 1997.

113. Vankan, W. J., J. M. Huyghe, J. D. Janssen, and A. Huson. A 3-D finite element model of blood perfused rat gastrocnemius medialis muscle. Eur JMorph 34: 19-24, 1996.

114. Vankan, W. J., J. M. Huyghe, J. D. Janssen, and A. Huson. Poroelasticity of saturated solids with an application to blood perfusion. Int J Engng Sci34: 1019-1031, 1996.

115. Vankan, W. J., J. M. Huyghe, J. D. Janssen, A. Huson, W. J. G. Hacking, and W. Schreiner. Finite element analysis of blood flow through biological tissue.Int J Engng Sci 35: 375-385, 1997. 
116. Vankan, W. J., J. M. Huyghe, D. W. Slaaf, C. C. v. Donkelaar, M. R. Drost, J. D. Janssen, and $A$. Huson. Finite element simulation of blood perfusion in muscle tissue during compression and sustained contraction. Am J Physiol 273: H1587-H1594, 1997.

117. Vankan, W. J., J. M. Huyghe, C. C. van Donkelaar, M. R. Drost, J. D. Janssen, and A. Huson. Mechanical blood-tissue interaction in contracting muscles: a model study. J Biomech 31: 401-409, 1998.

118. Vis, M. A., P. Sipkema, and N. Westerhof. Modeling pressure-area relations of coronary blood vessels embedded in cardiac muscle in diastole and systole. Am J Physiol 268: H2531H2543, 1995.

119. Willems, M. E., and P. A. Huijing. Mechanical and geometrical properties of the rat semimembranosus lateralis muscle during isometric contractions.J Biomech 27: 1109-1118, 1994.

120. Willems, M. E. T., and P. A. Huijing. Effect of heterogeneity of mean sarcomere length in different fibres: effects on length range of active force production in rat muscle.Eur / App/ Physiol Occup Physiol 68: 486-496, 1994.

121. Wisnes, A., and A. Kirkebo. Regional distribution of blood flow in calf musles of rat during passive stretch and sustained contraction. Acta Physiol Scand 96: 256-266, 1976.

122. Woittiez, R. D., P. A. Huijing, H. B. K. Boom, and R. H. Rozendal. A three-dimensional muscle model: a quantified relation between form and function of skeletal muscles.JMorph 182: $95-113,1984$.

123. Zajac, F. E. Muscle and tendon: properties, models, scaling, and application to biomechanics and motor control. Crit Rev Biomed Eng 17: 359-411, 1989.

124. Zuurbier, C. J. A. J. Everard, P. Van der Wees, and P. A. Huijing. Length-force characteristics of the aponeurosis in the passive and active muscle condition and in the isolated condition. J Biomech 27: 445-453, 1994.

125. Zuurbier, C. J., and P. A. Huijing. Changes in geometry of actively shortening unipennate rat gastrocnemius muscle. J Morphol218: 167-180, 1993.

126. Zuurbier, C. J., and P. A. Huijing. Influence of muscle geometry on shortening speed of fibre, aponeurosis and muscle. / Biomech 25(9): 1017-1026, 1992.

127. Zuurbier, C. J., and P. A. Huijing. Influence of muscle shortening on the geometry of gastrocnemius medialis muscle of the rat. Acta Anat Basel 140: 297-303, 1991. 


\section{SUMMARY}

Because any arbitrary movement of any part of the body is the result of skeletal muscle activity, proper muscle functioning is of major importance for the quality of life. Numerical and experimental research shows the importance of spatial effects to skeletal muscle mechanical functioning, which means that local effects inside a muscle can affect its behavior elsewhere. Yet, for the sake of simplicity, this is often neglected in experiments or numerical models. This thesis addresses spatial effects of skeletal muscle mechanics in two field of research. The first general aim regards the interaction between contraction and perfusion, the second objective relates to muscle deformation during isometric contraction. Both are studied using a combination of animal experiments and numerical simulations, using a finite element model of perfused contracting skeletal muscle.

The commonly most accepted hypothesis regarding the interaction between contraction and muscle perfusion is the vascular waterfall theory. This theory ascribes the amount of perfusion during contraction to the difference between arterial and tissue pressure, rather than to the arterialvenous pressure gradient. This phenomenon is inevitably present during highly controlled pressure box experiments. To determine the interaction between perfusion and contractions, total arterial inflow and venous outflow during contractions of various strengths are measured. These variables closely relate to each other. Total arterial inflow decreases $90 \%$ during forceful contractions, whereas vascular volume only decreases $0.21 \%$ of total muscle volume. The numerical model is used to interpret these experimental findings. Total flow signals, calculated by the model, are compared to experiments for validation. Simulations qualitatively agree with experiments; similar characteristics, particularly in the venous flow signal, are obvious. However, the model output quantitatively differs from experiments. From the combination of experiments and simulations it is concluded that the vascular volume change during contraction comes largely to the account of venous blood volume. This localized effect in the hierarchical dimension of the vascular tree, the large impact of contraction on perfusion and the minimal volume change are in agreement with the vascular waterfall theory.

This vascular waterfall theory is mainly studied using pressure boxes. These are used to apply an external pressure to a muscle, which results in a homogeneous tissue pressure. However, during contractions, intramuscular pressure is distributed. The interaction between distributed pressure during contraction and the behavior of the vascular waterfall is further explored using the finite element model. Simulations show that the 
spatial distribution of pressure and flow in separate blood compartments depends on the number of arteries and veins that is modeled. This difference is caused by spatial interaction between the heterogeneous intramuscular pressure and venous pressure during contraction. Therefore, extrapolation of the generally accepted vascular waterfall principles to local intramuscular phenomena is disallowed in situations in which geometrical effects may be important.

To study the importance of geometrical effects for muscle mechanics, muscle deformation is considered. Muscle deformation relates to muscle function, because sarcomere length directly depends on deformation. In an experimental study, longitudinal and transverse strains at the muscle surface during isometric contraction are determined from threedimensional displacement measurements of fluorescent markers that are attached to the muscle surface. With decreasing total muscle length, longitudinal and transverse strain in aponeurosis and superficial muscle fibers increase. Aponeurosis transverse strain increases twice as much as transverse strain in superficial muscle fibers with decreasing muscle length. Further, it was shown that transverse strain in superficial muscle fibers compensates for differences in longitudinal strain during contractions at different muscle lengths. Although surface area change of the aponeurosis during contraction depends on muscle length, the surface area change of superficial muscle fibers equals $-11 \%$, independent of initial muscle length. Assuming incompressible muscle tissue, strain in the direction perpendicular to the muscle surface equals $11 \%$. Because transverse strain maximally reaches $9 \%$, it is concluded that superficial muscle fibers change aspect ratio during isometric contraction, whereas the degree of this change depends on initial muscle length. For the difference between the behavior of muscle and aponeurosis transverse strain and for the changing aspect ratio with changing muscle length, it is concluded that three-dimensional deformation depends on muscle length and should be accounted for when studying muscle mechanics at different lengths.

To allow comparison of deformation measurements with numerical simulations, anatomically realistic geometrical information must be included in the model. Validation of model deformation with experimental data benefits from comparison of contractions under various conditions. One applicable variation regards the initial muscle length changes, studied in chapter 4 . This study shows that for such purpose, an actual threedimensional muscle geometry should be used. Therefore, a detailed reconstruction of the rat triceps surae muscle based on histological slides has been generated, from which a three-dimensional mesh of the medial gastrocnemius muscle is derived. This mesh can be used in finite element simulations of skeletal muscle contraction. However, because the mesh lacks accurate fiber direction data, proper comparison between simulated 
and experimentally determined deformation is not possible. For practical considerations, simulations in other parts of the thesis use a twodimensional mesh that represents the midsagittal longitudinal section through the medial gastrocnemius muscle.

Searching for suitable ways to include three-dimensional muscle fiber directions in a reconstruction, MRI techniques are given attention. Diffusion tensor imaging is validated as to measure fiber directions within $5^{\circ}$ accuracy. In combination with high-resolution MRI, a mesh of the anterior tibialis muscle, including realistic muscle fiber directions, an internal and an external aponeurosis, is generated. As an example of the use of this method in mechanical studies, internal deformation of this particular muscle during contraction is calculated, which is hard to determine experimentally.

In general, the present thesis studies spatial effects of skeletal muscle functioning regarding perfusion and deformation. It is shown how muscle perfusion can be influenced by geometrical effects, whereas deformation is essentially a three-dimensional phenomenon. Finite element simulations and experiments are used in close harmony in this thesis. Both experiments and simulations benefit from this interaction. The model was used to interpret experiments (chapter 2) and to calculate phenomena that cannot be measured experimentally (chapter 3) or with great efforts, using advanced and expensive techniques like tagging MRI. On the other hand, experiments were invoked to validate the model (chapter 2) and to draw general conclusions regarding the use of comparable models (chapter 4). Further, experiments support the model by providing accurate geometrical input (chapters 5 and 6). 


\section{SAMENVATTING}

Aangezien iedere willekeurige beweging van lichaamsdelen wordt veroorzaakt door activiteit van skeletspieren, is goed functioneren hiervan van groot belang voor het welzijn. Onderzoek naar het functioneren van spierweefsel toonde onder andere aan, dat ruimtelijke effecten een rol spelen in het mechanisch functioneren. Dit betekent dat locale effecten in een spier de werking ervan op een andere plaats kunnen beïnvloeden. Dusondanks worden deze effecten vaak verwaarloosd, om interpretatie van experimenten of simulaties met numerieke modellen te vereenvoudigen. In dit proefschrift worden deze ruimtelijke effecten van het mechanisch skeletspier functioneren nader bestudeerd in twee verschillende onderzoeksgebieden. Het ene gebied omvat de ruimtelijke interactie tussen contractie en perfusie, terwijl het tweede ingaat op spierdeformatie tijdens isometrische contractie. Beide onderdelen worden bestudeerd door dierexperimenten te combineren met numerieke simulaties. Deze simulaties worden uitgevoerd met een eindige elementen model van doorbloed, contraherend skeletspierweefsel.

De meest gangbare hypothese om de interactie tussen contractie en doorbloeding te beschrijven, is de veneuze waterval theorie. Deze veronderstelt dat niet het verschil tussen arteriële en veneuze druk, maar die tussen arteriële en intramusculaire druk de doorbloeding van skeletspier bepaalt tijdens contractie. Deze hypothese is onderbouwd door middel van experimenten met drukkamers, waarmee artificieel een hoge, homogene druk opgelegd kan worden aan het weefsel. Om doorbloeding tijdens een contractie te bestuderen, is de totale arteriële en veneuze flow door de kuitspier van een rat gemeten tijdens contracties van verschillende kracht. De mate van perfusie blijkt sterk afhankelijk van contractiespanning. De doorbloeding vermindert $90 \%$ tijdens krachtige contracties, terwijl het totale bloed volume in de spier maar met maximaal $0,21 \%$ afneemt. Ter interpretatie van deze data is het genoemde numericke model gebruikt. Gemeten en berekende flow signalen komen kwalitatief overeen, waarbij met name het veneuze flowsignaal opvallende overeenkomsten vertoont tussen experiment en simulatie. Kwantitatief verschillen simulaties echter van experimentele resultaten. Uit de combinatie van beide methoden is geconcludeerd, dat het kleine uitgeperste volume met name uit de venen komt. Dit gelokaliseerde effect in de hiërarchische richting, de grote afname van perfusie en de kleine verandering in volume, zijn in overeenstemming met de veneuze waterval theorie.

Deze waterval theorie is hoofdzakelijk experimenteel bestudeerd met behulp van drukkamers. Hierbij wordt een externe druk aan de spier 
opgelegd, die een homogene weefseldruk tot gevolg heeft. Echter, tijdens een contractie is de intramusculaire druk heterogeen verdeeld. De invloed van deze drukverdeling op het gedrag van de veneuze waterval is verder bestudeerd met het model. Simulaties lieten zien dat de ruimtelijke verdeling van druk en flow in verschillende bloedcompartimenten afhankelijk is van het aantal arterièn en venen dat wordt gemodelleerd. De oorzaak is een ruimtelijke interactie van de heterogene weefseldruk met de verdeling van de veneuze druk tijdens contractie. Dit heeft tot gevolg dat extrapolatie van de veneuze waterval theorie naar lokale, intramusculaire fenomenen niet zonder meer is toegestaan naar situaties waarin ruimtelijke effecten een rol spelen.

Het belang van ruimtelijke effecten voor spier mechanica is verder bestudeerd met betrekking tot deformatie tijdens contractie. Deformatie van spierweefsel is van belang voor het spierfunctioneren, omdat lengteverandering van spiervezels direct gerelateerd is aan sarcomeerlengte. Vervorming in de lengte- en in de dwarsrichting van het oppervlak van de kuitspier van een rat zijn experimenteel bepaald door de drie-dimensionale verplaatsing te meten van fluorescente markers, die op het oppervlak zijn aangebracht. De resultaten lieten zien, dat met kortere spierlengte zowel lengte- als dwarsrek tijdens contractie toenemen. Dwarsrek in de peesplaat neemt twee keer zo snel toe als die in de spiervezels met afnemende spierlengte. Verder is aangetoond dat dwarsrekken in de oppervlakkige spiervezels volledig compenseren voor de verandering in lengterek bij verschillende spierlengtes. Hoewel de oppervlakte verandering van de peesplaat afhankelijk is van de spierlengte, neemt het oppervlak van de oppervlakkige spiervezels onafhankelijk van de spierlengte met $11 \%$ af. Wanneer het volume van een spier tijdens contractie constant wordt verondersteld, moet de diameter van oppervlakkige spiervezels in de richting loodrecht op het oppervlak met $11 \%$ toenemen. Omdat dwarsrek maximaal $9 \%$ is, is de conclusie getrokken dat de verhouding tussen de kleinste en de grootste diameter van de $\mathrm{dwarsdoorsnede} \mathrm{door} \mathrm{een} \mathrm{gemiddelde} \mathrm{spiervezel} \mathrm{tijdens} \mathrm{isometrische}$ contractie verandert. De mate waarin deze vervorming optreed hangt af van de initiële spierlengte. Vanwege de verschillende invloed van spierlengte op dwarsrek in spiervezels en peesplaat, en omdat de vormverandering van de dwarsdoorsnede door spiervezels afhangt van initiële spierlengte, moet de drie-dimensionale vervorming ook in modellen worden meegenomen wanneer het effect van spierlengte op het spierfunctioneren wordt bestudeerd.

Om gesimuleerde vervormingen te toetsen aan experimentele metingen, is geometrisch realistische model input nodig. Modelvalidatie kan dan bijvoorbeeld plaatsvinden door spierdeformatie tijdens contractie onder verschillende condities te vergelijken met experimentele metingen. De 
contracties op verschillende spierlengtes, zoals uitgevoerd in hoofdstuk 4, zijn daarvoor geschikt. Uit dat hoofdstuk blijkt, dat voor een dergelijke vergelijking een drie-dimensionale geometrie nodig is. Daarom werd een drie-dimensionale reconstructie van de kuitspieren gemaakt uit histologische coupes. Een eindige elementen mesh van de $\mathrm{m}$. gastrocnemius medialis werd daaruit afgeleid, om te gebruiken in simulaties. Echter, omdat het in deze reconstructie niet mogelijk was om ook spiervezelrichtingen te bepalen, zijn vergelijkingen van deformatie tussen simulatie en experiment niet goed mogelijk. Vanuit praktische overwegingen is niet deze drie-dimensionale mesh, maar een daarvan afgeleide, twee-dimensionale representatie van het midsagittale vlak van de $\mathrm{m}$. gastrocnemius medialis, gebruikt in simulaties in andere delen van dit proefschrift.

Een methode waarmee wel de drie-dimensionale vezelrichtingen van diverse biologische weefsels kunnen worden bepaald is diffusie gewogen MRI. Deze techniek is in dit proefschrift gevalideerd voor skeletspier, waaruit bleek dat spiervezelrichtingen binnen een nauwkeurigheid van 5 graden kunnen worden bepaald. In combinatie met hoge-resolutie MRI werd vervolgens een model van de $\mathrm{m}$. tibialis anterior gemaakt, met anatomisch correcte vezelrichtingen en een interne en een externe peesplaat. Om het succes van de methode te illustreren, is een simulatie van een contractie met deze geometrie uitgevoerd, waarbij interne spierdeformatie wordt getoond.

Concluderend kan worden gezegd, dat ruimtelijke effecten van het functioneren van skeletspierweefsel met betrekking tot doorbloeding en deformatie in dit proefschrift zijn bestudeerd. Er is aangetoond, dat doorbloeding beïnloed kan worden door geometrische effecten en dat spierdeformatie tijdens contractie in essentie een drie-dimensionaal fenomeen is. Voor deze conclusies is een combinatie van modelsimulaties en experimenteel onderzoek nodig geweest. Het model is gebruikt om experimenten te interpreteren (hoofdstuk 2) en om effecten te berekenen, die niet of nauwelijks experimenteel kunnen worden bepaald (hoofdstuk 3). Daarnaast is het model getoetst met experimenten (hoofdstuk 2) en zijn op basis van experimentele metingen algemene conclusies getrokken, die betrekking hebben op vergelijkbare modellen (hoofdstuk 4). Tenslotte ondersteunen de experimenten het numerieke werk door het aanleveren van geometrisch juiste invoer yoor simulaties (hoofdstuk 5 en 6). 


\section{PUBLICATIONS}

\section{PAPERS}

1) C.C. van Donkelaar, M.R. Drost, H. van Mameren, C.F. Tuinenburg, J.D. Janssen, A. Huson, Three-dimensional reconstruction of the rat triceps surae muscle and finite element mesh generation of the gastrocnemius medialis muscle. Eur / Morphol34(1), 31-37 (1996).

2) W.J. Vankan, J.M. Huyghe, D.W. Slaaf, C.C. van Donkelaar, M.R. Drost, J.D. Janssen, A. Huson, Finite element simulation of blood perfusion in muscle tissue during compression and sustained contraction. Am J Physiol 42(3), H1587-H1591, 1997.

3) W.J. Vankan, J.M. Huyghe, C.C. van Donkelaar, M.R. Drost, J.D. Janssen, A. Huson, Mechanical blood-tissue interaction in contracting muscles: a model study. J Biomech 31(5), 401-410, 1998.

4) C.C. van Donkelaar, M.R. Drost, L.J.G. Kretzers, P.H.M. Bovendeerd, L.M.A. Lataster, K. Nicolay, J.D. Janssen, A validation of the diffusion tensor imaging technique for measurement of skeletal muscle fiber directions. J Anat 194(1), 79-88, 1999.

5) C.C. van Donkelaar, P.J.B. Willems, A.M.M. Muijtjens, M.R. Drost, Skeletal muscle transverse strain during isometric contraction at different lengths. J Biomech, 1999 (accepted).

\section{ABSTRACTS}

1) R. Huiskes, C.C. van Donkelaar, K.J. Jepsen, H. Weinans, S.A. Goldstein, E.H. Burger, The mechanical consequences of mineralization in fetal bone. 41st Annual meeting, Othopaedic Research Society, February 1316 1995, Orlando, Florida.

2) C.C. van Donkelaar, M.R. Drost, H.W.M. van Straaten, F.W. Prinzen, J.D. Janssen, A. Huson, A method to measure blood flow distribution in contracting skeletal muscle under controlled mechanical conditions. Eur J Physiol 430(4 suppl), R60 (1995). Abstracts of the First FEPS Congress, September 9-12 1995, Maastricht, The Netherlands.

3) C.C. van Donkelaar, M.R. Drost, H.W.M. van Straaten, F.W. Prinzen, J.D. Janssen, A. Huson, Measurement of 3D blood flow distribution in contracting skeletal muscle, using microspheres. 2nd European workshop on the Auorisicnt microsphere method, September 28-29 1995, Munich, Germany. 
4) M.R. Drost, C.C. van Donkelaar, L.J.G. Kretzers, K. Nicolay, J.D. Janssen, Does diffusion weighted MRI measure muscle fibre direction? 10th Conference of the European Society of Biomechanics, August 28-31 1996, Leuven, Belgium.

5) C.C. van Donkelaar, M.R. Drost, W.J. Vankan, J.M. Huyghe, J.D. Janssen, H. Kuipers, Blood perfusion of contracting skeletal muscle: a comparison between a finite element model and experiments. 1st International Interdisciplinary Conference on Cardiovascular Medicine, Surgery, Science, and Mechanics, June 6-9, 1997, Washington, DC, USA.

6) C.C. van Donkelaar, Does diffusion-weighted MRI measure fibre direction? In vivo NMR - present and future, September 27 1996, Utrecht, The Netherlands.

7) M.R. Drost, C.C. van Donkelaar, L.J.G. Kretzers and K. Nicolay. A comparison of local muscle fibre direction measured by diffusion tensor imaging (DTI) with muscle fibre direction as determined in an actual section. Proceedings 5th Scientific Meeting International Society of Magnetic Resonance in Medicine, April 12-18 1997, Vancouver, pp. 1717.

8) C.C. van Donkelaar, J.M. Huyghe, J.D. Janssen, M.R. Drost, Interaction between heterogeneous tissue pressure distribution and muscle perfusion. 1999 ASME Summer Bioengineering Conference, June 16-20, 1999, Big Sky, Montana. 


\section{DANKWOORD}

Het is af... op dit moment rolt het (als het goed is) uit de printer. De' laatste dingen nog opruimen, opzoeken wat ik wil bewaren, buro leegmaken en de computer opschonen. Die is al door een ander geconfisceerd. Mijn stuel staat opeens in de lig-stand en ik heb de neiging om even achterover te gaan liggen en uit te rusten van de afgelopen paar maanden waarin alles tegelijk leek te gebeuren. Maar dan gaat de telefoon. "Ik ben net thuis, heb langer doorgewerkt. Zal ik nog wachten, of zal ik al vast beginnen met eten koken?" is de strekking van het bekend voorkomende gesprek. "Nee, ik kom er zo aan", is het antwoord, "alleen nog even....".

Uiteindelijk trek ik ruim twee uur later de deur achter me dicht en sluit hem voor de laatste keer af, het manuscript stevig onder de arm geklemd. Natuurlijk moet ik ook het secretariaat afsluiten, want met het afnemend aantal dagen die je als aio nog te gaan hebt, ben je vaker als laatste aanwezig.

"Promoveren doe je niet alleen", staat in veel proefschriften, gevolgd door een opsomming van de mensen die bij het onderzoek hebben ondersteund. Natuurlijk is dat zo, en zullen een aantal namen zeker worden genoemd. Maar toch voelt het op een of andere manier symbolisch als je op je laatste dag als laatste de deur afsluit en alleen nog de nachtportier groet, die je inmiddels maar al te goed kent.

In gedachten verzonken loop ik naar buiten met een doos erfenissen uit het aio-tijdperk. Het manuscript kan naar de beoordelingscommissie. Nu alleen nog maar een dankwoord schrijven en een voorkant ontwerpen. "Dankwoord kort en zakelijk houden", denk ik uitgeblust. Maar gelijk daarna besef ik dat ik zeker niet al die mensen tekort wil doen die zich hebben ingespannen om een stukje van mijn onderzoek van de grond te krijgen.

Natuurlijk moet ik beginnen met het bedanken van de promotoren Ilarm Kuipers en Jan Janssen, die het mogelijk hebben gemaakt om dit werk uit te voeren. Datzelfde geldt ook voor Toon Huson, die de eerste twee jaren promotor was, maar samen met het voorzitterschap van de vakgroep $B W$ ook het promotorschap aan Harm overdeed.

Ik ben vooral veel dank verschuldigd aan co-promotor Maarten Drost. De vele discussies die we aan tafel en in het lab hadden (soms tot 2:30 's nachts, zeer tot verbazing van de portier) hebben mij veel wijzer gemaakt.

Natuurlijk heb ik ook veel geleerd van Jacques Huyghe, die mij als enige kon uitleggen waar Jos Vankan het nu toch weer over had, terwijl datzelfde 
ongeveer even zo vaak andersom gebeurde; mijn kennis van eindige elementen en poreuze media is voor $90 \%$ te danken aan hun geduld.

Op verschillende momenten tijdens mijn onderzoek droegen inhoudelijk met name Frits Prinzen, Hennie van Straaten en Klaas Nicolay bij, terwijl de praktische hulp van vooral Paul Willems en Arno Lataster, maar ook van vele anderen, onmisbaar is geweest.

En over inhoudelijk gesproken... hoeveel uren discussie zou er gevoerd zijn tussen aio's? Het eerste jaar met kamergenote Hennie, drie jaar lang met altijd aanwezige Josv, gedurende bijna vijf jaar met continu behulpzame Matthijs, en de laatste twee jaar met ruling Gors? Maar daarnaast zijn de minder inhoudelijke, al dan niet serieuze discussies met Harold, Gerard H, Gerard R, Lars, Bolletje en uiteindelijk kamergenote Pimi toch minstens zo belangrijk geweest. Zo niet voor de hoofdstukken, dan toch zeker voor enkele stellingen. En waar zou de vakgroep zijn zonder de ondersteuning van Harry en Desiree en de verhalen van Gerrit?

$\mathrm{Na}$ vandaag zullen alleen nog toekomstig kamergenote Marielle en systeembeheerders Leo en Patrick met de stress van het maken van de uiteindelijke versie van het proefschrift geconfronteerd worden. Tenslotte wacht de paranimfen Eddy en Bas de taak, om mij op het moment supreme op te peppen. Op dit moment lijkt dat wel heel moeilijk.

Thuisgekomen zet ik mijn fiets in de schuur en zie voorzichtig een hoofd tussen de gordijnen door gluren. Het eten is natuurlijk al lang klaar, maar ze heeft nog even op mij gewacht. En op dat moment besef ik dat het leven naast wetenschap nog zoveel meer te bieden heeft, dat vrienden nodig zijn om je daar steeds weer aan te herinneren, dat ouders onmisbaar zijn vanwege hun onvoorwaardelijke steun en hulp, en dat ik dit en zoveel meer niet zonder haar had kunnen bereiken. Hoe kan ik dat beter laten blijken dan door dit proefschift te beginnen en eindigen met de woorden voor Nanda. 



\section{Curriculum Vitae}

René van Donkelaar was born in Ermelo, the Netherlands, on November 1, 1970 and grew up in Ugchelen, where he finished high school in 1988. His interests in sports, biology and science were bundled in the study Human Movement Sciences at the University of Nijmegen, where he graduated in December 1993. He performed his practical training in the field of biomechanics at the Department of Orthopaedics in Nijmegen. Studying the mechanical behavior of a hip prosthesis was his first introduction to (bio)mechanical engineering, followed by a study on mechanical stimuli of fetal bone growth. He started his Ph.D. studies on skeletal muscle mechanics, which resulted in this thesis, on January 1 , 1994 at the department of Movement Sciences of the Maastricht University in cooperation with the faculty of Mechanical Engineering of the Eindhoven University of Technology. Since November 1, 1998, he is working at the design of an intervertebral disc implant at the Eindhoven University of Technology.

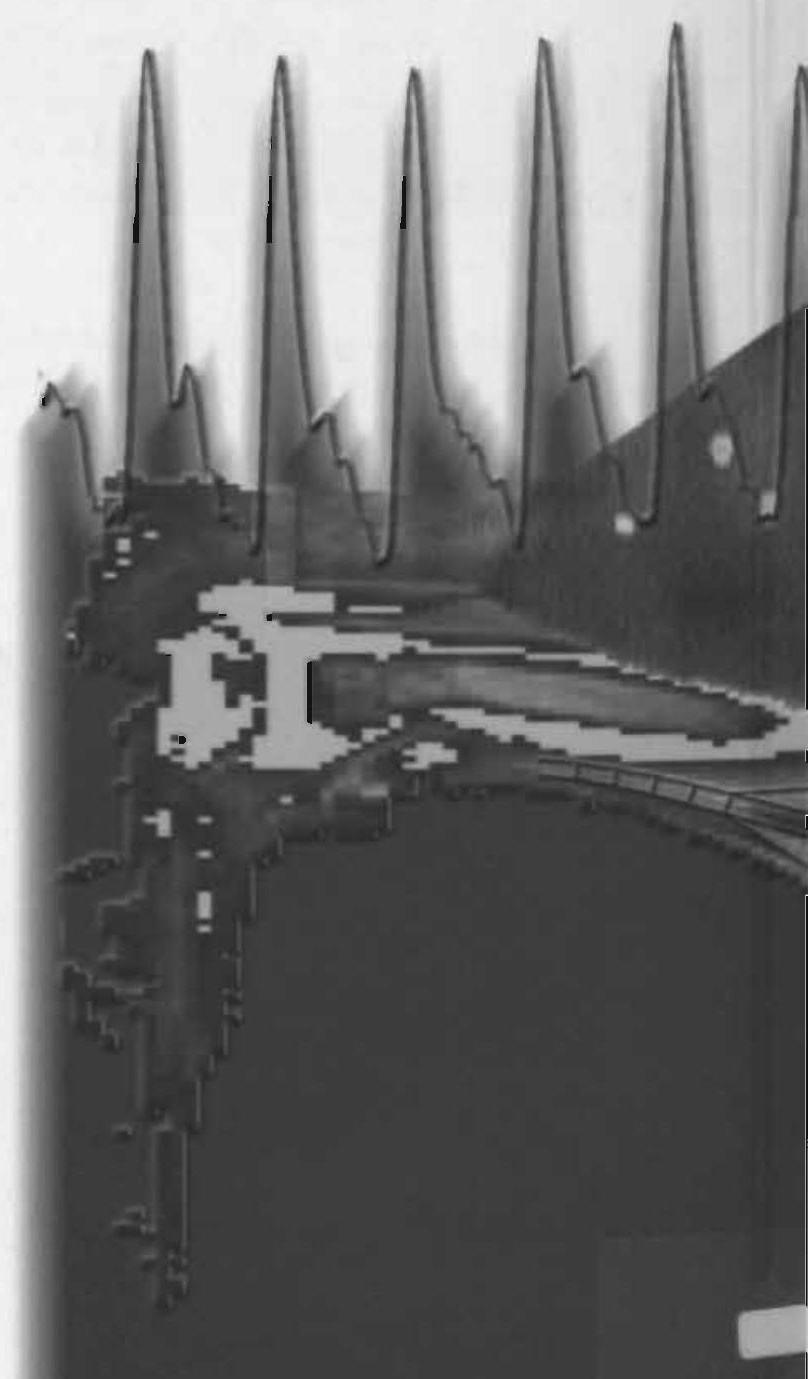

